\title{
PERFORMANCE ANALYSIS OF COGNITIVE RADIO NETWORKS WITH DIVERSITY AND FADED CHANNELS
}

\author{
by \\ SATtar J. Hussain \\ BSc, University of Baghdad, Baghdad, Iraq, 1983 \\ MSc, University of Baghdad, Baghdad, Iraq, 1992
}

\author{
A dissertation \\ presented to Ryerson University \\ in partial fulfillment of the \\ requirements for the degree of \\ Doctor of Philosophy \\ in the Program of \\ Electrical and Computer Engineering
}

Toronto, Ontario, Canada, 2013

(C)Sattar J. Hussain 2013 
I hereby declare that I am the sole author of this dissertation. This is a true copy of the dissertation, including any required final revisions, as accepted by my examiners.

I authorize Ryerson University to lend this dissertation to other institutions or individuals for the purpose of scholarly research.

I further authorize Ryerson University to reproduce this dissertation by photocopying or by other means, in total or in part, at the request of other institutions or individuals for the purpose of scholarly research.

I understand that my dissertation may be made electronically available to the public. 
Performance Analysis of Cognitive Radio Networks with Diversity and Faded Channels Doctor of Philosophy 2013

Sattar J. Hussain

Electrical and Computer Engineering

Ryerson University

\begin{abstract}
This dissertation presents new approaches for cognitive radio networks that combat fading effects and improve detection accuracy. We propose an advance framework for performance analysis of cooperative spectrum sensing over non-identical Nakagami- $m$ fading channels. Specifically, we investigate the detection accuracy of a relay-based cognitive radio network and derive new exact and approximated closed-form expressions for average detection probability and average false alarm probability for two diversity combining schemes; equal gain combining and selection combining. The dissertation presents novel approximation methods to mitigate the difficulties in modeling non-identical channel fading. We also investigate the convergence rate of infinite-series expressions and propose to use a powerful acceleration algorithm that allows for the termination of the series with a finite number of terms.

A detect-amplify-and-forward strategy is proposed to mitigate bandwidth requirements of relaying local observations to a fusion center. The end-to-end performance of a relay-based cooperative spectrum sensing over independent identically distributed
\end{abstract}


Rayleigh fading channels is also investigated in this dissertation. Specifically, we aim to incorporate sensing time, end-to-end SNR, and end-to-end channel statistic into the performance analysis of cooperative CR networks. We also propose a cluster-based cooperative spectrum sensing approach to overcome the bandwidth limitations of the reporting links. The approach reduces the number of reporting terminals to a minimal reporting set and replaces the global fusion center by a local fusion center to mitigate the destructive channel conditions of global relaying channels. A new approach is proposed to select the location of the local fusion center using the general center scheme in graph theory.

We aim to show that multipath fading on relaying channels yields similar performance degradations as multipath fading on sensing channels. With the detect-amplify-and forward strategy, refraining the heavily faded relays improves the detection accuracy. A gain of $3 \mathrm{~dB}$ is achieved by switching from amplify-and-forward strategy to detect-amplifyand-forward strategy with 3 cooperative users. Compared to the non-cooperative spectrum sensing, a gain of up to $8 \mathrm{~dB}$ is achieved with 4 cooperative users and equal gain combining receiver. Similar experimental set up but with selection combining receiver, a gain of $5 \mathrm{~dB}$ is achieved. 


\section{Acknowledgements}

First, I would like to express my deepest appreciation to Professor Xavier Fernando, for his guidance, continuous support, and encouragement which are essentials through my research. His advice and feedback about my research have greatly enhanced and strengthened the work. I thank him for all the time and energy he has invested into my research.

Secondly, I would like to thank my committee chair, Professor Lian Zaho and my committee members, Professor Ngok-Wah Ma and Professor Issac Woungan for their valuable comments and suggestions.

Finally, I would like to express my gratitude to my wonderful family for their understanding and patient. Without their support it might be impossible to complete my $\mathrm{PhD}$ study. They have stood by me in everything I have done providing constant support, encouragement, and love. 


\section{Dedication}

This Doctoral dissertation is dedicated to my wife Siham, my sons: Ali and Mustafa, and my daughter Rahaf who have supported me all the way since the beginning of my study. 


\section{Table of Contents}

Abstract ............................. ii

Acknowledgements . . . . . . . . . . . . . . . . . . . v

Dedication . . . . . . . . . . . . . . . . . . vi

Table of Contents . . . . . . . . . . . . . . . . . . vii

List of Tables . . . . . . . . . . . . . . . . . . . . . viii

List of Figures . . . . . . . . . . . . . . . . . . . . . ix

List of Acronyms . . . . . . . . . . . . . . . . . . . ix

List of Symbols . . . . . . . . . . . . . . . . . . . xii

List of Appendices . . . . . . . . . . . . . . . . . . . . . xiii

1 Introduction 1

1.1 Background . . . . . . . . . . . . . . . . . . 1

1.2 Spectrum Sensing Challenges _. . . . . . . . . . . . . . . . . 3

1.3 Motivation . . . . . . . . . . . . . . . . . . . 6

1.4 Related Works . . . . . . . . . . . . . . . . . . 9 9

1.5 Contributions . . . . . . . . . . . . . . . . . . . . 11

2 Cognitive Radio Technologies $\quad 15$

2.1 Cooperative Spectrum Sensing . . . . . . . . . . . . . . . . 15

2.2 Relaying (reporting) Channels . . . . . . . . . . . . . . . . 18

2.3 Diversity Combining Techniques . . . . . . . . . . . . . . . . 20

2.4 Spectrum Sensing over Fading Channels . . . . . . . . . . . . . 23

2.5 Primary Signal Detection . . . . . . . . . . . . . . . . . . 24

2.6 Energy Detection . . . . . . . . . . . . . . . . . . 26

2.7 Cluster-Based Spectrum Sensing . . . . . . . . . . . . . . . . . 28

3 Amplify-and-Forward Strategy over Non-Identical Fading Channels 31

3.1 Introduction . . . . . . . . . . . . . . . . . . . . . . . 31 
3.2 Spectrum Sensing Model . . . . . . . . . . . . . . . . . . . . 33

3.2.1 Single-Relay System . . . . . . . . . . . . . . . . . . 35

3.2 .2 Multi-Relay System . . . . . . . . . . . . . . . . . 38

3.2.2.1 Selection Combining Scheme . . . . . . . . . . . . 38

3.2.2.2 Equal Gain Combining Scheme . . . . . . . . . . . . . . 41

3.2.3 Convergence Acceleration . . . . . . . . . . . . . . 45

3.2 .4 Approximated Analysis . . . . . . . . . . . . . . . . . . 46

3.3 Performance Evaluation . . . . . . . . . . . . . . . . 51

4 Detect-Amplify-and-Forward Strategy over Non-Identical Fading Channels $\quad 59$

4.1 Introduction . . . . . . . . . . . . . . . . . . . . . 59

4.2 Spectrum Sensing Model . . . . . . . . . . . . . . . . . 60

4.2 .1 Single-Relay System . . . . . . . . . . . . . . . . 60

4.2 .2 Multi-Relay System . . . . . . . . . . . . . . . . . 63

4.2 .3 Approximated Analysis . . . . . . . . . . . . . . . . 67

4.3 Performance Evaluation _. . . . . . . . . . . . . . . . . . 69

5 End-to-End Performance over Rayleigh Fading Channels $\quad \mathbf{7 4}$

5.1 Introduction . . . . . . . . . . . . . . . . . . . . . . 74

5.2 Spectrum Sensing Model . . . . . . . . . . . . . . . . 74

5.2.1 End-to-End Cooperative Spectrum Sensing Model . . . . . . . . . 76

5.3 Performance Evaluation _. . . . . . . . . . . . . . . . 81

6 Cluster-Based Cooperative Spectrum Sensing $\quad 87$

6.1 Introduction . . . . . . . . . . . . . . . 87

6.2 Network Model . . . . . . . . . . . . . . . . . . . . . . 88

6.3 The Clustering Algorithm . . . . . . . . . . . . . . . . . . . 91

6.4 Cluster-Based Cooperative Spectrum Sensing . . . . . . . . . . . . . 98

6.5 Throughput Performance . . . . . . . . . . . . . . . . . . 101

6.6 Performance Evaluation . . . . . . . . . . . . . . . . . . . . . 104

7 Conclusions and Future Works $\quad 110$

7.1 Conclusions . . . . . . . . . . . . . . . . . . . 110

7.2 Suggestions for Future Works . . . . . . . . . . . . . . 113 


\section{APPENDICES}

$\begin{array}{ll}\text { A } & 116\end{array}$

A.1 Derivation of $\bar{P}_{f_{i}}$ for a single-relay system . . . . . . . . . . . . . 116

A.2 Derivation of $\bar{P}_{f_{S C}} \ldots \ldots \ldots \ldots \ldots \ldots \ldots \ldots \ldots$

A.3 Derivation of $\bar{P}_{f_{E G C}} \ldots \ldots \ldots \ldots \ldots \ldots \ldots \ldots$

B $\quad 120$

B.1 Derivation of $\bar{P}_{f}^{D A F} \ldots \ldots \ldots \ldots \ldots \ldots \ldots \ldots \ldots$

B.2 Derivation of the approximated $\bar{P}_{f}^{D A F} \ldots \ldots \ldots \ldots \ldots$. . . . . . . 121

$\begin{array}{ll}\mathrm{C} & 122\end{array}$

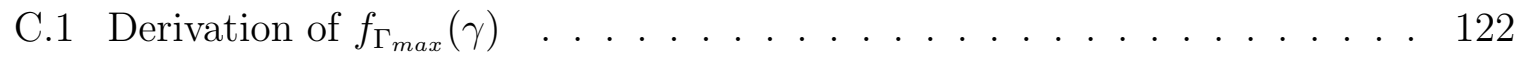

C.2 Derivation of $\bar{P}_{d}$ over i.i.d Rayleigh channels . . . . . . . . . . . . . . 123

$\begin{array}{ll}\text { References } & 126\end{array}$ 


\section{List of Tables}

3.1 Converging point required to evaluate $\bar{P}_{d}$ for SC and EGC schemes. $L=3$,

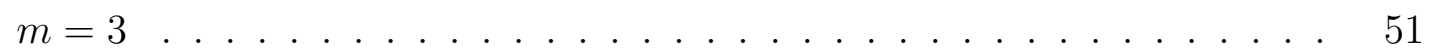

3.2 Converging point required to evaluate (3.16) and (3.28) with $\varepsilon$-algorithm.

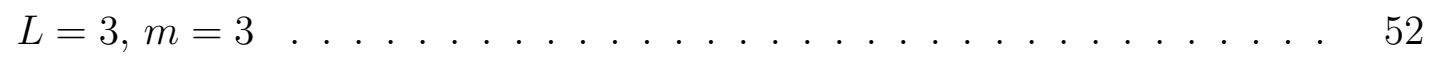




\section{List of Figures}

1.1 Hidden terminal and receiver uncertainty problems . . . . . . . . . . 4

1.2 Periodic spectrum sensing structure . . . . . . . . . . . . . . . 5

2.1 Centralized cooperative spectrum sensing system. . . . . . . . . . . . 17

2.2 Two-user cooperation in cognitive radio . . . . . . . . . . . . . . . . . 17

2.3 Typical energy detection receiver . . . . . . . . . . . . . . . . . . . 27

2.4 Sensing round structure in cluster-based spectrum sensing . . . . . . . . 29

2.5 Cooperative spectrum system strategies a) centralized system b) distributed system . . . . . . . . . . . . . . . . . . . . . 30

3.1 Computation structure of Wynn's $\varepsilon$-algorithm . . . . . . . . . . . 47

3.2 Comparison of analytical results of expression (3.47) with Monte Carlo simulations. $L=3, m=3 \ldots \ldots \ldots$. . . . . . . . . 54

3.3 Comparison of analytical and simulated $\bar{P}_{d} . \quad P=5 \mathrm{~dB}, L=3, m=2$, $\bar{\gamma}_{s}=0 \mathrm{~dB} \ldots \ldots \ldots \ldots \ldots \ldots \ldots$

3.4 Complementary ROC curves at different relaying channel conditions. $P=$ $5 \mathrm{~dB}, L=3, \bar{\gamma}_{s}=0 \mathrm{~dB} \ldots \ldots \ldots \ldots 6$

3.5 Average detection probability for different level of sensing channel SNR. $P=5 \mathrm{~dB}, L=3, m=2 \ldots \ldots \ldots \ldots 6$

3.6 Average detection probability vs. relay maximum transmitting power, $\mathrm{P}$. $L=3, \lambda=20, \bar{\gamma}_{s}=0 \mathrm{~dB} \ldots \ldots \ldots \ldots$. . . . . . . . . 58

3.7 Average detection probability vs. relay power constraint, P. $m=3, \lambda=$ $20, \bar{\gamma}_{s}=0 \mathrm{~dB} \ldots \ldots \ldots \ldots \ldots \ldots \ldots$

4.1 Comparison of analytical results of expressions (4.22) and (4.26) with Monte Carlo simulations for $L=3, m=2, P=5 \mathrm{~dB}$, and $\bar{\gamma}_{s}=0$ dB. . . . . . . . . . . . . . . . . . 70

4.2 ROC curves of the energy detector with $m=2, P=5 \mathrm{~dB}$, and $\bar{\gamma}_{s}=0 \mathrm{~dB} . \quad 70$ 
4.3 Detection performance of AF and DAF strategies for different diversity scenarios with $m=2, P=5 \mathrm{~dB}$, and $\bar{\gamma}_{s}=0 \mathrm{~dB} \ldots \ldots . \ldots . . \ldots 72$

4.4 Detection performance of $\mathrm{AF}$ and DAF strategies for different fading sce-

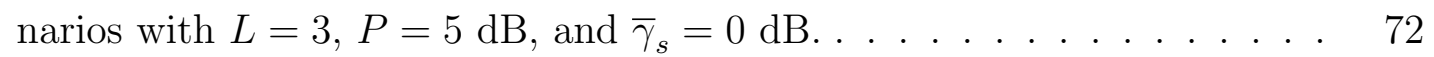

4.5 Detection performance of AF and DAF strategies for different power constraints with $\lambda=5, \mathrm{~m}=2$, and $\bar{\gamma}_{s}=0 \mathrm{~dB} \ldots \ldots \ldots . \ldots . \ldots 73$

5.1 Comparison of average detection probability in equation (5.12) with Monte Carlo simulation for $\mathrm{L}=1,2$, and 3. $\bar{\gamma}_{s}=\bar{\gamma}_{r}=5 d B, u=6 \ldots \ldots 2$

5.2 Comparison of approximated and exact Marcum_Q function. $u=6 . . \quad . \quad 83$

5.3 Average detection probability $\bar{P}_{d}$ vs. sensing Time for $\mathrm{L}=1,3$, and 5 .

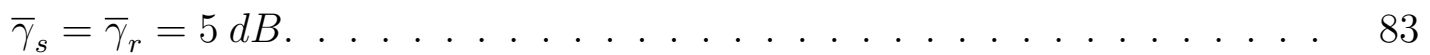

5.4 Average detection probability, $\bar{P}_{d}$, vs. $\bar{\gamma}_{r}$, for $\mathrm{L}=1,3$, and $5 . \bar{\gamma}_{s}=5 \mathrm{~dB}$, $u=8 \ldots \ldots \ldots \ldots \ldots \ldots \ldots$

5.5 Amount of fading vs. number of cooperative users. $\bar{\gamma}_{s}=10 \mathrm{~dB} . \ldots . \quad 85$

6.1 Maximum distance to the nearest cluster head . . . . . . . . . . . . . 95

6.2 Cluster-based cooperative spectrum sensing . . . . . . . . . . . . 97

6.3 fraction of single-node clusters vs cluster radius for 1, 2, 3-hop clusters . 105

6.4 Fraction of single-node clusters vs cluster radius under different node den-

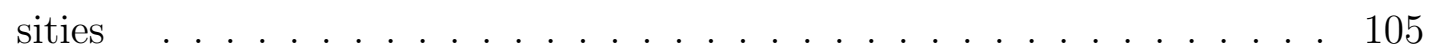

6.5 Cluster-based cooperative sensing performance with various cluster size,

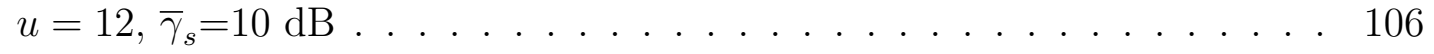

6.6 False alarm vs cluster size $u=12, \bar{\gamma}_{s}=10 \mathrm{~dB} \ldots \ldots \ldots$. . . . . . . 107

6.7 The normalized per-node throughput vs number of cooperative clusters,

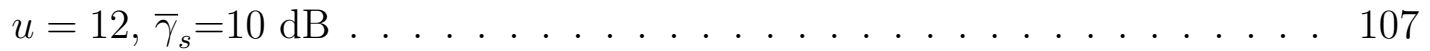

6.8 Per-node throughput performance of clustered and non-clustered network vs number of cooperative terminals, $u=12, \bar{\gamma}_{s}=10 \mathrm{~dB} \ldots \ldots . . . . . \quad 109$

6.9 The minimal dominating set vs cluster radius for 1 and 2-hop clustering . 109 


\section{List of Acronyms}

$\begin{array}{ll}\text { AF } & \text { Amplify-and-Forward } \\ \text { AoF } & \text { Amount of Fading } \\ \text { AWGN } & \text { Additive White Gaussian Noise } \\ \text { CF } & \text { Characteristic Function } \\ \text { CSI } & \text { Channel State Information } \\ \text { CSS } & \text { Cooperative Spectrum Sensing } \\ \text { CDF } & \text { Cumulative Distribution Function } \\ \text { CDMA } & \text { Code Division Multiple Access } \\ \text { CR } & \text { Cognitive Radio } \\ \text { CRC } & \text { Cyclic Redundancy Check } \\ \text { DAF } & \text { Decode-Amplify-and-Forward } \\ \text { DF } & \text { Decode-and-Forward } \\ \text { DSA } & \text { Dynamic Spectrum Access } \\ \text { EGC } & \text { Equal Gain Combining } \\ \text { i.i.d } & \text { Independent Identically Distributed } \\ \text { ISM } & \text { Industrial, Scientific and Medical } \\ \text { LOS } & \text { Line Of Sight } \\ \text { MDS } & \text { Minimal Dominating Set } \\ \text { MGF } & \text { Moment Generating Function } \\ \text { MRC } & \text { Maximal Ratio Combining } \\ \text { MSa/s } & \text { Mega Samples per Second } \\ \text { NP } & \text { Non-deterministic Polynomial-time } \\ \text { PDF } & \text { Probability Density Function } \\ \text { QoS } & \text { Quality of Service } \\ \text { RF } & \text { Radio Frequency } \\ & \end{array}$


ROC Receiver Operating Characteristic

RV Random Variable

$\mathrm{SC} \quad$ Selection Combining

SNR Signal-to-Noise Ratio

TDMA Time Division Multiple Access

UWB Ultra Wide Band 


\title{
List of Symbols
}

\author{
$\lambda \quad$ Decision threshold \\ $\theta \quad$ Primary user state \\ $\widehat{\theta} \quad$ Estimated primary user state \\ $\sigma_{Y}^{2} \quad$ Mean value of a power denoted by $Y$ \\ $m_{i} \quad$ Nakagami- $m$ fading parameter of the $i$ th link \\ $\Gamma$ (.) Gamma function \\ $\Gamma(.,$.$) \quad Incomplete gamma function$ \\ $\gamma_{s_{i}} \quad$ Signal-to-noise ratio of the $i$ th sensing link \\ $\gamma_{r_{i}} \quad$ Signal-to-noise ratio of the $i$ th relaying link \\ $\gamma_{e_{i}} \quad$ Instantaneous end-to-end SNR of the $i$ th relaying link \\ $\mathrm{E}\{\cdot\} \quad$ Expected or mean value of a RV \\ $\bar{g}_{i} \quad$ Expected value of the $i$ th relaying link \\ $\beta_{i} \quad$ Denotes $m_{i} / \bar{g}_{i}$ \\ $U(. ; . ;$.$) \quad Confluent hypergeometric function$ \\ $K_{v}() \quad$.$v -order modified Bessel function of the second kind$ \\ $Q_{u}(.,$.$) \quad Generalized Marqum-Q function of u$ degree of freedom \\ $U($.$) \quad Unit step function$ \\ $I_{u-1}($.$) \quad Modified Bessel function of (u-1)$ th order \\ $\mu \quad$ Arrival rate of Poisson distribution \\ $V \quad$ Set of vertices of a given graph \\ $\Xi \quad$ Set of edges or links between vertices in a given graph \\ $N_{\text {iso }} \quad$ Number of isolated nodes \\ $h_{p} \quad$ Number of transmission hops \\ $\mathcal{C} \quad$ Cluster subset \\ $v_{i}^{1} \quad$ Set of 1 -hop neighbors of the node $v_{j}$
}




\section{Appendices}

Appendix A . . . . . . . . . . . . . . . . . . 116

A.1 Derivation of $\bar{P}_{f_{i}}$ for a single-relay system . . . . . . . . . . . . . . . . . . . . . . . . . . . . .

A.2 Derivation of $\bar{P}_{f_{S C}} \ldots \ldots \ldots \ldots \ldots \ldots \ldots \ldots$

A.3 Derivation of $\bar{P}_{f_{E G C}} \ldots \ldots \ldots \ldots \ldots \ldots \ldots \ldots$

Appendix B . . . . . . . . . . . . . . . . . . . 120

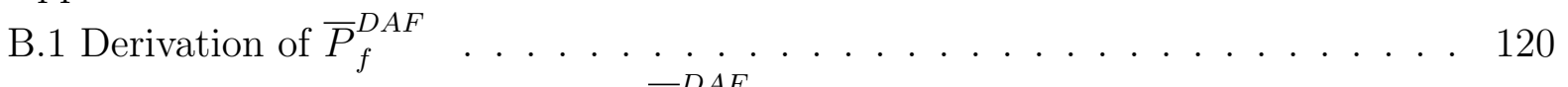

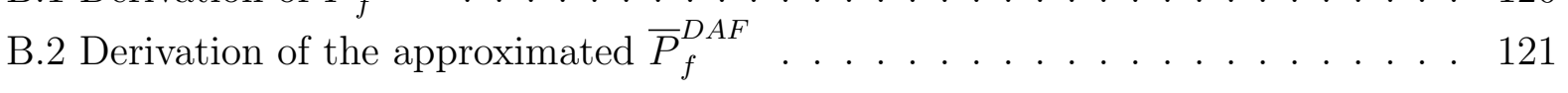

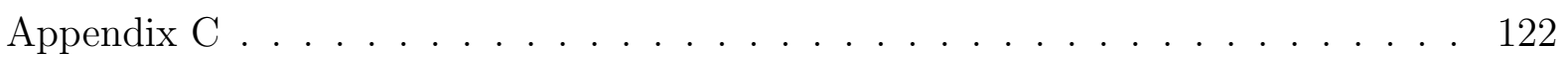

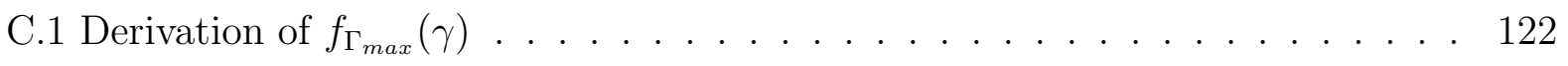

C.2 Derivation of $\bar{P}_{d}$ over i.i.d Rayleigh channels . . . . . . . . . . . . . . . 123 


\section{Chapter 1}

\section{Introduction}

\subsection{Background}

Cognitive radio (CR) technology arises to be an attractive solution to the spectral congestion problem by introducing opportunistic usage of the frequency bands that are not heavily occupied by licensed users $[1,2]$. CR concept will change the way the radio spectrum is shared and will require enabling technologies such as DSA, spectrum sharing, flexible spectrum policy, and improved spectrum sensing techniques [3].

Since CR users are considered lower priority or secondary users of a spectrum allocated to a primary user which the licensed entity, a fundamental requirement is to avoid interference to the primary users in their vicinity [3]. Through CR technology, secondary users periodically monitor the spectrum for vacant channels, and communicate among themselves using these vacant channels even though these channels are originally owned 
by the primary users. The cognitive capability of a CR enables real time interaction with its environment to determine appropriate communication parameters and adapt to the dynamic radio environment [4].

Spectrum sensing is considered the key step in CR systems and the most challenging issue [5]. Spectrum sensing, in general, performs the following two functionalities [6]: 1) Locating spectrum holes so that CR users can communicate in the absence of the primary network, 2) Monitoring the spectrum band and preparing to abandon the channel immediately as soon as the primary network resumes its operation.

Typically, spectrum sensing performance is determined by two significant parameters namely, probability of detection and probability of false alarm [7]. Probability of detection determines the detection accuracy; it indicates how well the primary user is protected from harmful interference caused by the secondary users' transmissions. On the other hand, false alarm probability determines sensing efficiency; it refers to the probability of white-spaces that are misclassified as occupied channels. From the secondary users point of view, a lower probability of false alarm means more spectrum access opportunities and higher network throughput. While from the primary network's perspective, a higher detection probability means more protection against interferers. Depending on which probability is of interest, that one is fixed and the other one is optimized [8].

CR users perform spectrum sensing either individually or cooperatively. In the individual spectrum sensing, also known as non-cooperative spectrum sensing, each CR observes its local radio environment and determines the presence of the primary user 
transmission and accordingly identifies the current spectrum availability [6]. On the other hand, cooperative spectrum sensing allows CR users to exchange their local observations or their sensing results and cooperatively infer the availability of spectrum holes.

\section{$1.2 \quad$ Spectrum Sensing Challenges}

The basic assumption of the spectrum sensing is that the locations of the primary receivers are unknown due to the lack of signalling between primary users and CR users. Therefore, CR terminals have to only rely on weak primary transmitter signals based on local observations [9]. Accordingly, CR receiver sensitivity must be higher than the highest primary receiver sensitivity by a large margin [4]. Moreover, the transmitter detection model cannot prevent a hidden terminal problem. The hidden terminal problem is considered as a critical challenging issue in the spectrum sensing [4]. It arises when the secondary user fails to detect the primary transmitter due to either deep fading/shadowing or receiver uncertainty as shown in Fig. 1.1. In this case, the CR cannot sense the presence of the primary user signal, and thus it is allowed to access the channel while the primary user is still in operation.

Multipath fading is a main obstacle for CR users to achieve reliable communications [10]. Under channel fading or shadowing a low received signal strength does not necessarily imply that the primary system is not active or located out of the CR's interference range as the signal may be experiencing a deep fade or is being heavily shadowed 


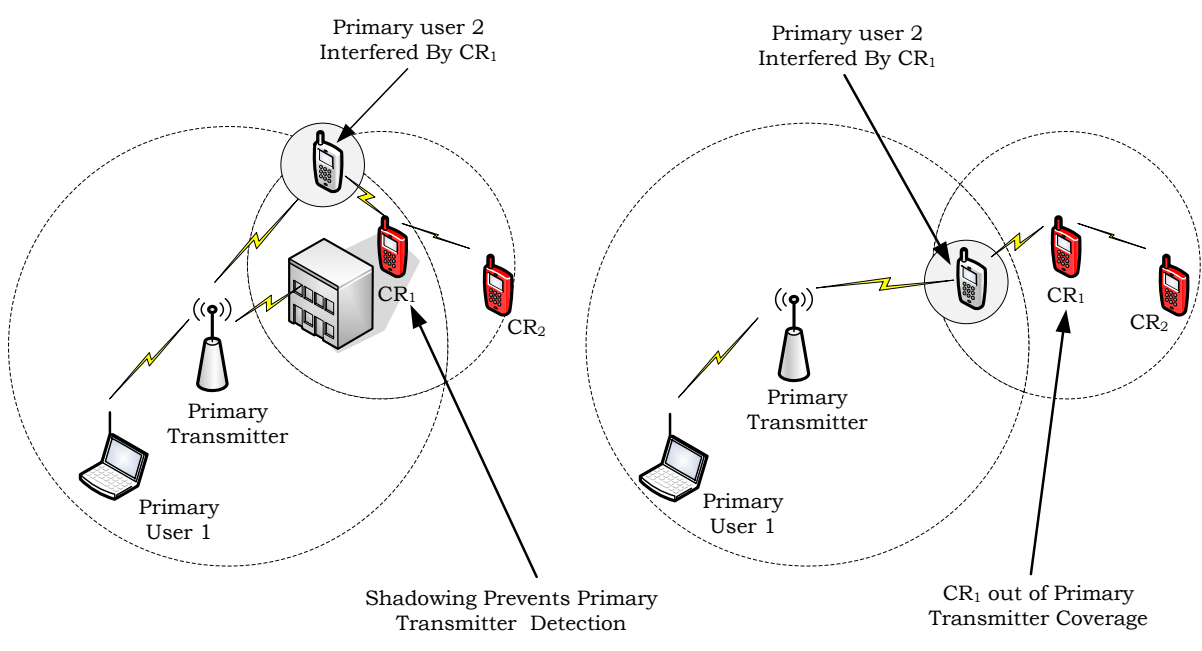

Figure 1.1: Hidden terminal and receiver uncertainty problems

by obstacles [11]. Hence, a performance analysis for both coherent or noncoherent detections over fading channels requires knowledge of the fading envelope statistics and will be considered in detail in the following chapters.

CR users should monitor the spectrum continuously. However, in reality the receiver's RF front-end cannot differentiate between the primary user signals and CR user signals $[3,12]$. Thus, CR users can not transmit and sense at the same time. This necessitates a periodic sensing scheme where sensing and transmitting are alternating in a periodic manner with separate observation and transmission periods [13]. Fig. 1.2 illustrates the basic structure of spectrum sensing cycles in CR networks. The periodic sensing structure introduces another design challenge [13]. Interference avoidance depends on the sensing accuracy which is in turn determined by the observation time. Since the transmission of CR users is not allowed during the observation time, the transmission time is reduced which inevitably reduces the spectrum usage efficiency. 


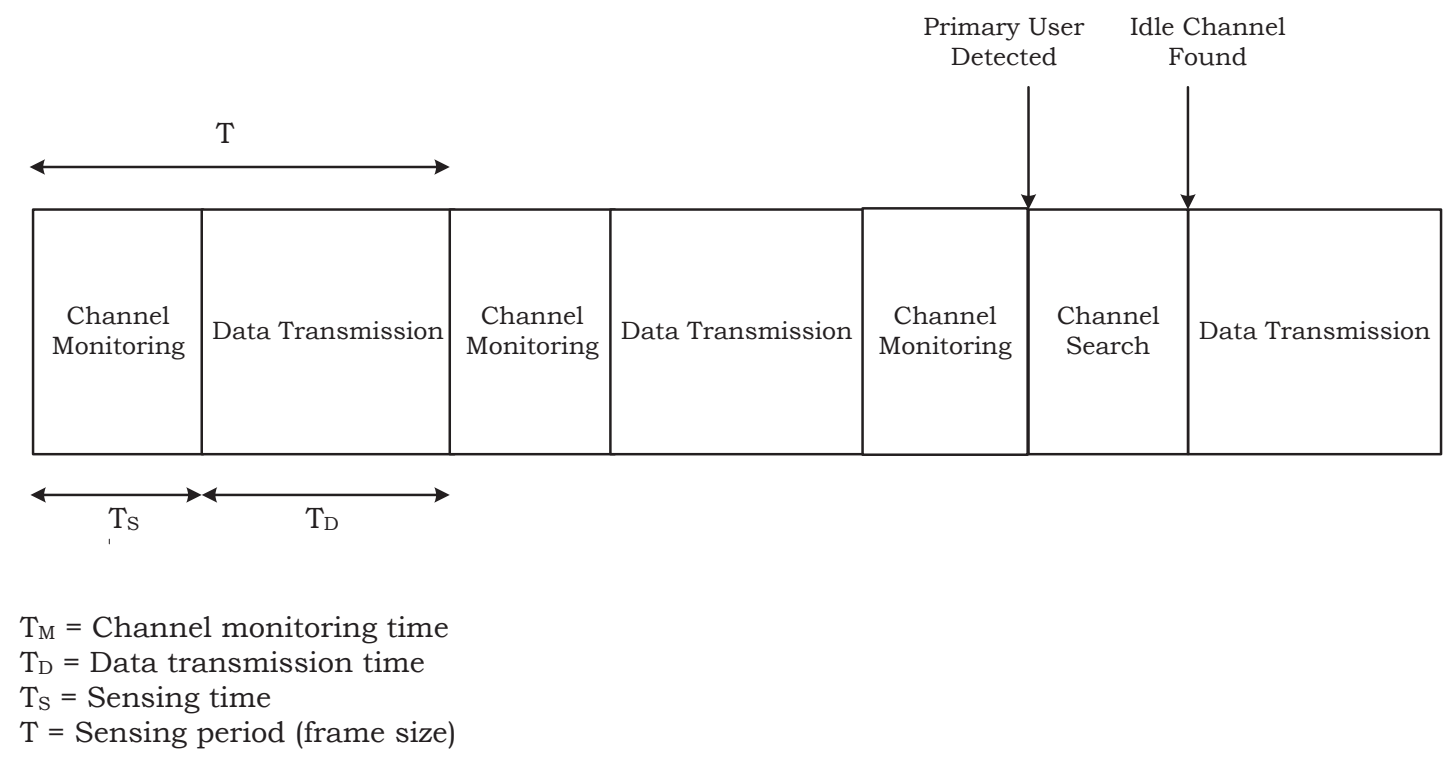

Figure 1.2: Periodic spectrum sensing structure

Spectrum sensing is also challenged by the fact that the available spectrum holes show different characteristics which vary over time. In order to describe the dynamic nature of CR networks, each spectrum hole should be characterized by considering not only the time-varying radio environment but also the primary user activity and the spectrum band information such as operating frequency and bandwidth [4]. Most CR research assumes that the primary user activity is modeled by exponentially distributed interarrivals $[9,13-15]$. In this model, the primary user traffic is modeled as a two state birth-death process. An ON (busy) state represents the period used by primary users and an OFF (idle) state represents the unused period [16]. Since each user's arrival is independent, each transition follows the Poisson arrival process and the length of the ON and the OFF periods are exponentially distributed. 


\subsection{Motivation}

Reliable detection of the existence of primary users is a primary requirement for the minimization of interference to existing primary networks $[17,18]$. In a real communication environment, the local sensing performance of individual users may severely degrade due to deep fading/shadowing [19-21]. Therefore, individual spectrum sensing is unreliable and prone to errors. However, spectrum sensing can be significantly improved by allowing different users to share their local sensing observations and to cooperatively decide on the licensed spectrum occupancy.

By exploiting the diversity provided by associated radios, CSS improves the overall detection sensitivity without imposing higher sensitivity requirements on the individual CRs $[19,22,23]$. It has been shown that a network of cooperative cognitive radios, which experience different channel fading conditions from the target, would have a better chance of detecting the primary radio if the individuals' local sensing are jointly combined at a base station $[24,25]$.

The merit of CSS primarily lies in the achievable space diversity brought by the sensing channels, namely, sensing diversity gain provided by the multiple CRs. Even though one CR may fail to detect the primary signal, there are still many chances for other CRs to detect it. Another merit of cooperative spectrum sensing is the mutual benefit brought forward by communicating with each other to improve sensing performance and system agility. When a CR is far away from the primary user, the received signal is too weak and the user takes a long time to sense its presence. However, when a 
CR located nearby the primary user acts as a relay, the signal of the primary user can be reliably detected by the far user in a very short time thereby improving system agility [26, 27]. Moreover, by allowing multiple CRs to cooperate in spectrum sensing, the hidden terminal problem can also be addressed [3]. While a cooperative approach provides a more accurate sensing performance, it causes adverse effects on resourceconstrained networks due to the additional operations and overhead traffic $[4,28]$.

Obviously, CSS will go through two successive channels [29]: First, the sensing channels which are the links between the primary user and the CRs, and second, the relaying channels which are the links between the CRs and the fusion center. Most results on CSS (see $[19,23,30-32]$ and references therein) have retained the typical assumptions of classical decentralized soft-fusion framework; i.e., the effect of the wireless channel is taken into account only on the sensing channel and is overlooked on the relaying link. Most of these studies have dealt with the CR network as a single-hop communication system, ignoring the effect of relaying channel conditions. Therefore, the noise accumulation that effects typical relaying protocols is neglected. Even when faded relaying channels are considered (see e.g., $[26,27,33,34])$, the fading model on both sensing and relaying links are oversimplified.

Typically, the wireless propagation characteristics of the relaying channels and the relaying mechanism play a significant role in CSS performance. Specifically, the achievable detection accuracy of the cooperative spectrum sensing methods depends on the channel fading conditions, relaying protocol, and the employed combining scheme [35]. 
Importantly, the relaying channels convey noise to the destination, which is why it is necessary to incorporate its propagation characteristics into the performance analysis. The difference between a conventional single-hop system and a relay-based dual-hop system is that the noise in the single-hop system is independent of the channel statistics while the noise in the dual-hop system is not. Motivated by these facts, this dissertation aims to offer an accurate framework for the analysis and design of CSS using the detection accuracy as a performance measure and taking into account realistic channel conditions and system operations.

The relaying channels are usually bandwidth limited [3]. If every user transmits the real value of its observation, a large relaying bandwidth is required which may not be available. The bandwidth limitations of the relaying channel are addressed by this dissertation in two ways: i) only the users with reliable information are allowed to report their local observations, ii) instead of local observations, users are allowed to report a binary decision to the common receiver. However, for large networks, the aforementioned approaches may not be enough to overcome the bandwidth limitations since the bandwidth of the relaying link increases with the number of relays. Hence, a clustering approach is proposed in this study. Clustering is one of the most efficient techniques used to reduce the bandwidth required to report data to a fusion center [36-40]. It is used widely as a hierarchical approach of topology management in Ad-Hoc wireless networks [36, 41-43]. However in CR networks, clustering must consider the fact that the available channel sets are changing temporally and spatially. Furthermore, the cluster sizes and cluster loca- 
tions may change when CR's neighborhood changes with each new operating frequency. Consequently, clustering in CR networks is performed according to channels topology instead of nodes topology [44, 45]. Specifically, a CR node forms a cluster on an available channel and invites adjacent nodes to join its cluster if the same channel is available in their channel sets.

\subsection{Related Works}

There has been an active research in exploring CSS performance in cognitive radio networks. This section discuss the up-to-date research relevant to our work.

In $[13,24,45-48]$, the authors investigated CSS performance under typical assumptions of error-free relaying channels and ignored channel impairments due to fading and shadowing conditions. In $[30,49]$, the authors investigated the performance of an energy detector over wireless channels with composite multipath fading and shadowing effects. In both studies, the proposed CSS models are quantified based on the detection probability as a performance measure. However, the authors overlooked the relaying channels and dealt with the cooperative system as a single-hop network. Similarly, the relaying links are overlooked in [50]. This work presents a performance analysis of energy detector over multipath fading for a system with antenna diversity reception.

To the best of the author's knowledge, very few works have considered realistic modeling of the relaying channels in the CSS approaches proposed for CR networks, e.g. $[33,34,51]$. In [51], the authors considered the error effect on reporting decisions to 
a fusion center, investigating the problems of using CSS over independent and identically distributed (i.i.d.) Rayleigh fading channels. A DF relaying protocol is employed and a logic-based decision fusion policy is considered as a combining technique. The authors in [33] considered the AF relaying protocol for a CR network with the reported channels subjected to log-normal shadowing. However, closed form expressions for the average detection probability and the average false alarm probability are not given in this study. In [34], a distributed model is proposed for CSS networks assuming that channel statistics follow an exponential distribution. The final expressions of the detection and false alarm probabilities are presented by integral forms that need to be solved by numerical integration.

To show that the cooperation of two secondary users or more increases the overall agility, the authors in [26] and [27], proposed two cooperative schemes. A common receiver is used in the first one to exchange the local sensing information among two users while a totally distributed cooperative scheme is used in the second one for multiuser networks. For the multiuser case, the authors developed a pairing protocol which ensures asymptotic agility gain with probability 1 . The AF protocol is employed in both studies to exchange the local sensing information. The study assumes a fixed cooperation model which may fail due to the mobility of the paired CR users.

The authors in [47], propose that secondary users with higher detection probabilities constantly act as relays to help those with lower detection probabilities. In [46], the authors showed that if all secondary users cooperate in the network does not necessarily 
achieve the optimum performance. Instead, optimum performance can be achieved by cooperating users with the highest signal to noise ratio. The number of cooperating users to obtain the optimum detection and false alarm probabilities is computed at a secondary base station.

A weighed-CSS scheme using clustering is proposed in [52]. In the clustering setup, the most active user with the largest instantaneous reporting channel gain is to be selected as a cluster head. The authors assumed a reporting channel that is subjected to Rayleigh fading. In [53], a cluster-based method is proposed for CSS over imperfect relaying channels. To decrease the reporting errors introduced by the fading channels, user selection diversity is exploited in each cluster. The largest instantaneous reporting channel gain is used as a selection criteria for cluster heads election. The clustering algorithm proposed in this dissertation differs from the above previous works in two important aspects. Firstly, we employed a fully distributed network, whereas the approaches in $[52,53]$ are proposed for centralized networks. Secondly, the MDS scheme is used to reduce the number of reporting terminals to a minimal reporting set.

\subsection{Contributions}

This thesis investigates a number of problems challenging the cooperative spectrum sensing in both centralized and distributed systems and proposes new techniques to address these challenges. In particular:

- In a different way to the typical assumptions of i.i.d fading channels, we analyze 
the CSS performance over a non-identical Nakagami- $m$ fading channels.

- We present a general framework for analysis and design of dual-hop CR networks that employs realistic system operations.

- We investigate two CSS strategies, namely, AF and DAF. The energy detection is considered to allow soft-fusion (data-fusion) policy to be used at the receiver. Softfusion is known to achieve optimal diversity and allows for keeping the complexity of local radios at a low level since only analog forwarding is required by them. However, the optimum performance comes at the cost of bandwidth requirements of the relaying links. To mitigate this problem, we propose the DAF strategy to refrain those users with unreliable information from forwarding their local measurements to the fusion center.

- We develop a statistical approach to derive the detection probability and the false alarm probability. To the best of our knowledge, this is the first time closed-form expressions are derived for CSS systems with non-identical fading channels on both sensing and relaying links.

- Based on harmonic mean of two random variables, we obtain a PDF for the endto-end SNR. The end-to-end statistic is then used to quantify the performance of the CSS using the detection accuracy and the amount of fading as performance measures.

- We investigate the convergence rate of the infinite series that appears in the derived 
expressions and propose to use a powerful acceleration algorithm that allows the series termination with a finite number of terms.

- We present novel approximation methods to mitigate the difficulties in modeling non-identical channel fading.

- We investigate a cluster-based CSS approach for cognitive networks with a large number of CR users. We also propose new criteria to select local fusion centers. Instead of selecting the radio with the largest instantaneous channel gain as a local fusion center, we propose to use the aggregate channel gain as a selection criteria.

- We present a systematic way to find the optimal number of cooperative clusters. The MDS clustering approach is used to obtain a minimal set of clusters that covers all the users operating in the field while keeping the network connected.

The cooperative sensing is performed as follows: each CR radio carries out local sensing within a specified sensing time period and then acts as a relay to forward the local sensing to the fusion center. Based on the combined inputs, the fusion center decides whether the primary network is active or not. The secondary users operate in a fixed TDMA scheme where the sensing and transmission phases are alternating periodically. The spectrum sensing phase consists of two time slots: In the first slot, all CRs listen to the primary user signal over the shared spectrum band, and in the second slot, each CR amplifies and relays its local sensing to the fusion center by a maximum transmission power constraint. The fusion center reports the cooperative decision back to the CRs 
at the end of the second slot. The statistic of the combined signal is determined by the amplification factor of the CR relay, the combining technique, and the fading conditions.

All findings are substantiated via analytical computations using MATHEMATICA-8 software package [54] and verified through Monte Carlo simulations. 


\section{Chapter 2}

\section{Cognitive Radio Technologies}

\subsection{Cooperative Spectrum Sensing}

Cooperative communications is an emerging and a powerful solution that can overcome the limitations of wireless systems $[55,56]$. The basic idea behind cooperative transmission is that the signal transmitted by a source to a destination, each employing single antenna, is also received by other terminals which are often referred to as relays [29]. The destination then combines the signals coming from the source and the relays, thereby creating spatial diversity by taking advantage of the multiple receptions of the same data at the various terminals and transmissions paths. More specifically, the presence of multiple radios helps to reduce the effects of severed multipath at a single radio since they provide the destination with multiple independent realizations of related random variables. With multiple realizations, the probability that all users see deep fades is very low [57]. 
For cooperative decisions, reporting channels (relaying links) are used by CR radios to report their local observations (soft-fusion) or their individual decisions (decision-fusion) to a secondary base station (fusion center). In this dual-hop cooperative network, the primary user stands for the source and the common receiver stands for the destination. The basic structure of this cooperative approach is shown in Fig. 2.1. In another approach of cooperative networks, secondary users with higher detection probabilities constantly act as relays to help those with lower detection probabilities [26, 27]. Such cooperation can reduce the detection time of the "weaker" user thereby improving the agility of the overall network. Fig. (2.2) shows a scenario of two-user cooperation. In the presence of more than two users, CR users can be grouped into pairs, in which one user acts as a relay for the other [27]. However, the performance of such fixed cooperation model will become worse when considering mobility of the CR users.

CSS can be implemented either in a centralized system or in a distributed system $[5,58]$. In the centralized method, a base-station works as a fusion center to gather all sensing information from the CR users and detect the spectrum holes. Cooperation among CR users in a centralized manner is usually coordinated by a fusion center through either decision-fusion or soft-fusion policies [59,60]. The centralized network architecture helps overcome the receiver uncertainty problem. With the transmitter detection, CR networks cannot avoid interference at the nearby primary user since the transmitter's detection relies only on local observations of CR users and does not consider the location information of the primary receiver [61]. Hence, in order to reduce the receiver's uncer- 


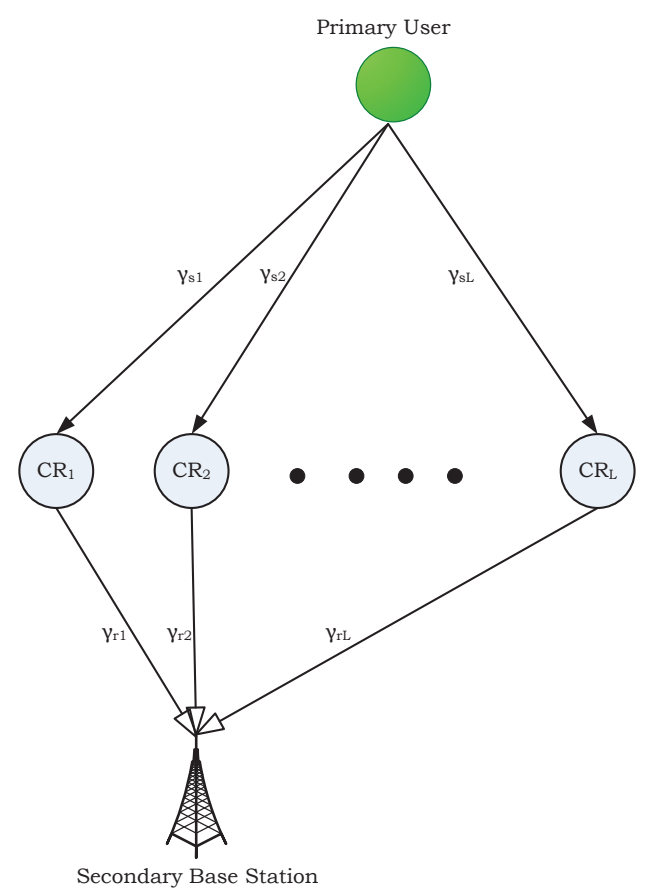

Figure 2.1: Centralized cooperative spectrum sensing system.

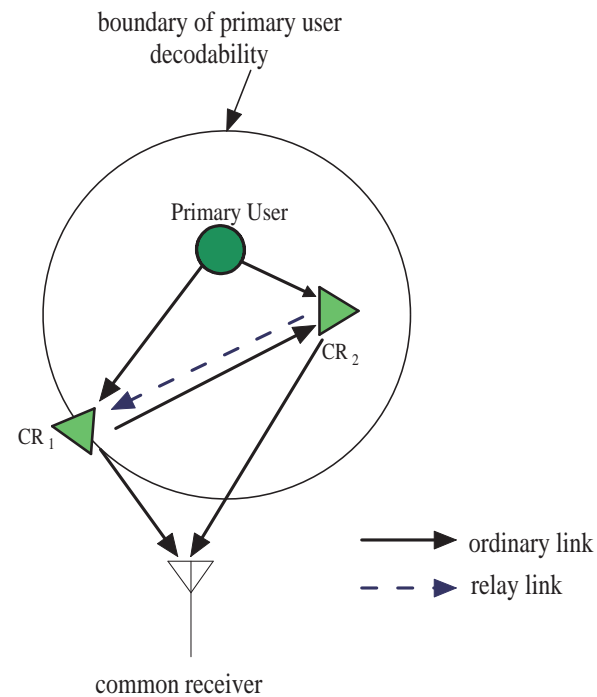

Figure 2.2: Two-user cooperation in cognitive radio 
tainty, CR networks require the base-station to collect sensing information from CR users inside its coverage area. Moreover, the centralized approach mitigates the limitation in sensing capabilities. For example, CR networks should be synchronized to schedule the spectrum sensing among all CR users. Thus, CR networks need to have a base-station to synchronize the scheduling such that all users can have the same sensing cycles and avoid interfere with sensing operations [13].

On the other hand, distributed solutions require an exchange of observations among CR users [62]. In the fully distributed system, all signal processing is done at the CR terminals and the results are available locally. Global results are then available at a local fusion center [63]. Distributed solutions are mainly proposed for cases where the construction of an infrastructure is not preferable $[9,62,64]$. Therefore, distributed networks lack centralized support, and hence must rely on local coordination to gather topology information [6]. A common cooperative scheme of distributed systems is forming clusters to share the sensing information locally [65].

\section{$2.2 \quad$ Relaying (reporting) Channels}

CSS either centralized or distributed, assume relaying channels for spectrum sharing [59, 64]. It is clear that relaying channels facilitate many spectrum sharing functionalities such as transmitter receiver handshake, communication with a fusion center, or sensing information exchange. However, due to the fact that CR network users are regarded as secondary users of the spectrum that they allocate, when a primary user chooses a 
channel this channel has to be vacated without interfering. This is also true for the relaying channels. In cooperative sensing architectures, the relaying channels can be implemented using different methodologies. These include a dedicated band, an unlicensed band such as ISM band, and an UWB underlay system $[3,66]$. Depending on the system requirements, one of these methods can be selected.

The relaying links provide more flexibility for designing advanced cooperative protocols. Specifically, the level of cooperation is determined by the bandwidth of the relaying channel. To this end, we can define two regimes of interest [11]:

- Low bandwidth relaying channels for energy detection and cyclostationary detection receivers: In this regime, it is realistic to assume that the CRs exchange decisions or summary statistics rather than long vectors of raw data.

- High bandwidth relaying channels: in this regime, CRs can exchange entire raw data and hence a sophisticated detection can be performed. In this scenario, it may be possible for tightly synchronized radios to collectively overcome poor detection scenarios.

When the relaying channel is jammed or unavailable, CR users should adapt to the new situation and use an alternative channel. For example, CR users may use a global control channel [67]. To facilitate the change over to the alternative relaying channel, efficient information sharing and coordination algorithms should be developed [68]. These algorithms must be designed to reduce overhead traffic caused by the cooperation process and should be robust to the temporal variations of relaying channels. 


\subsection{Diversity Combining Techniques}

A relayed transmission is a well-known technique that has the advantage of extending the coverage without using large power at the transmitter. Recently, it has gained a new actuality in the context of collaborative wireless communication systems [9, 27, 69], where relaying is used as a form of spatial diversity to overcome highly shadowed or deeply faded links. As a result, the signal from the source (primary user) to the destination (fusion center) propagates through two hops (links) in series [70]. Depending on the nature and complexity of the relays, dual-hop transmission systems can be classified into two main categories, namely, regenerative or non-regenerative systems. In regenerative systems, the relay fully decodes the signal that went through the first hop and retransmits the decoded version into the second hop [71]. This is also referred to as decode-and-forward or digital relaying [69]. On the other hand, non-regenerative systems use less complex relays that just amplify and forward the incoming signal without performing any sort of decoding. That is why it is sometimes referred to as amplify-and-forward or analog relays.

As a further categorization, relays in non-regenerative systems can in their turn be classified into two categories: 1) CSI-assisted relays, and 2)"blind" relays. Nonregenerative systems with CSI-assisted relays use instantaneous CSI of the first hop to control the gain introduced by the relay and as a result fix the power of the retransmitted signal. In contrast, systems with "blind" relays do not need instantaneous CSI of the first hop at the relay, but rather employ at these relays amplifiers with fixed gains 
and consequently result in a signal with variable power at the relay output. Although systems with such a type of blind relays are not expected to perform as well as systems equipped with CSI-assisted relays, their low complexity and ease of deployment make them attractive from a practical standpoint [71]. In cognitive radio applications, the CSI may be available to secondary users over a control channel or over a broadcast channel through an access point. Several such steps have recently been investigated $[72,73]$.

AF relaying allows a soft-fusion policy to be used by the receiver. The soft-fusion policy had been proven to achieve optimal diversity, providing a higher diversity gain and a better detection accuracy compared to the logic-based decision-fusion policy $[32,74,75]$. Moreover, it allows for keeping the complexity of local sensors at a low level since only analog forwarding is required by them $[26,27,31,34,74]$. This is true when radios are tightly synchronized to collectively overcome the SNRwall ${ }^{1}$. When the AF protocol is implemented, several diversity combining techniques can be used at the receiver. The object of diversity combining techniques is to make use of the several received signals to improve the realized SNR and combat the effect of the multipath fading [76]. Diversity combining relies on a simple principle. Namely, if a number of well-separated relays are used to relay the same signal, it is unlikely that all received signals at the fusion center fade at the same time. In general, the term " diversity system" refers to a system in which one has available two or more closely similar copies of some desired signal [76]. Diversity combining offers one of the greatest potentials for radio link performance improvement $[10]$.

\footnotetext{
${ }^{1}$ SNRwall is the weakest SNR below which a detector fails to reliably detect the signal
} 
Diversity schemes can be classified according to the type of combining employed at the receiver as MRC, SC, and EGC. The MRC combiner weighs the received signals with respect to their SNR and is known to be of high performance. However, MRC receiver's complexity is directly proportional to the number of diversity branches. The EGC equally weighs the diversity branches but it has the same undesirable feature of having the receiver complexity dependent on the number of diversity branches [77]. Conversely, the SC scheme selects only one relay which has the maximum channel gain among all the diversity branches. The combiner offers a simple receiver structure since it is simply performed by measuring the SNR of all the diversity branches.

On the other hand, DF relaying allows decision-fusion policy to be implemented at the receiver. It aims to minimize the overhead traffic and the bandwidth requirements by allowing each user to report only a single bit which represents its own decision. When decision-fusion policy is used; AND, OR or M-out-of-N methods can be used for combining information from different cognitive radios [46]. However, in practical systems, to reliably deliver one-bit sensing information actually involves a lot of overheads, including reliable channel coding, packet preamble, CRC bits etc. Typically, the number of cooperative users and the amount of information that each user should send to the fusion center determine the required bandwidth. For a large network, the required bandwidth may exceed the capability of the reporting channels, which may be alleviated through DF relaying. 


\subsection{Spectrum Sensing over Fading Channels}

Many urban and vehicular communication systems are subjected to fading caused by multipath propagation due to reflections, refractions and scattering by buildings and other large structures [78]. For primary user detection, flat fading yields the worst case performance since frequency selectivity provides multiple "looks" at the same signal [79]. Furthermore, in a composite fading/shadowing environment, apart from the multipath fading, wireless signals may undergo shadowing. While the multipath fading can be modeled as a Rayleigh, Rice or Nakagami distribution, shadowing process is typically modeled as a Log-normal distribution $[49,79]$ and the reference therein. In this environment the receiver does not average out the envelope fading due to multipath but rather reacts to the instantaneous composite multipath/shadowed signal [10]. This is often the scenario in congested areas with slow moving pedestrians and vehicles [80]. Therefore, some practical communication channels can be modeled as a multipath fading superimposed on Log-normal shadowing [49]. Due to difficulty of analyzing digital wireless communication systems over composite fading models, the shadowing effect is sometimes neglected in the literature. It is also important to keep in mind that shadowing is notoriously hard to model accurately and its statistics can vary with the deployment's environment [57]. 


\subsection{Primary Signal Detection}

Spectrum sensing techniques can be classified into three principal types [3]: matched filter detection, cyclostationary detection, and energy detection. When the noise is Gaussian and the signal has a known form, even with unknown parameters, the appropriate processing includes a matched filter or its correlator equivalent [81]. The matched filter is an optimal detector in AWGN channel since it maximizes the received SNR [82]. The main advantage of matched filter is that it needs less time to achieve high processing gain due to coherent detection [83]. However, the matched filter requires the synchronization between the primary user and the CRs [6]. Furthermore, CR users need to have different multiple matched filters dedicated to each type of the primary user signal, which increase the implementation cost and complexity [6].

Cyclosationary detection determines the presence of the primary user signals by extracting their specific features such as pilot signals, cyclic prefixes, symbol rate, spreading codes, or modulation types from its local observation [6]. The main advantage of the feature detection is its robustness to the uncertainty in noise power. If the signal of the primary user exhibits strong cysclostationary properties, it can be detected at very low SNR values by exploiting the information (cyclostationary features) embedded in the received signal $[6,84]$. Furthermore this detection approach can distinguish the primary signal from other CR users' signals over the same frequency band provided that the cyclic features of the primary user and the CRs' signals differ from each other [85]. However, cyclostationary detection is more complex to implement than the energy detection and 
requires a prior knowledge of the primary signal such as modulation format $[29,86]$.

Energy detector is a non-coherent detector that is simple to implement [30]. It is considered an optimal detector in case of unknown signal if the noise power is known [3]. Thus, the energy detector is widely considered as the local spectrum sensing detection method since the CRs have no prior knowledge about the primary signal due to the lack of interaction with the primary user. The reliability of the energy detector depends on the receiver's noise characteristics, the received signal strength, and the length of time that is used for the integration. While the energy detector is easy to implement and can be used without any prior knowledge of the primary signal, it still has some drawbacks. The first problem is that it has poor performance under low SNR conditions and will not be able to detect the signal reliably if the SNR is less than $S N R_{\text {wall }}[3,87]$. Also, the energy detector suffers from longer detection time compared to the matched filter detection [6]. Another challenging issue is the ability to differentiate the interference from other secondary users sharing the same channel and the primary user. For this reason, CR networks need to provide a synchronization over the sensing operations of all neighbors, i.e. each CR user should be synchronized with the same sensing and transmission schedules. Otherwise, CR users cannot distinguish the received signals from primary and CR users, and hence the sensing operations of the CR user will be interfered by the transmissions of its neighbors. Furthermore, the threshold used in energy detection depends on the noise variance, and small noise power estimation errors can result in significant performance loss [88].

The energy detector is employed in our study. We aim to investigate the problems 
of the energy detector and analyze its performance over multipath fading channels. The cooperative sensing may alleviate the problems of the energy detector and improve its performance. In cooperative sensing we rely on the variability of signal strength at various locations. We expect that a number of cooperative CRs with sensing information exchanged through a fusion center would have a better chance of detecting the primary user compared to individual sensing.

\subsection{Energy Detection}

Detection of a signal in the presence of noise requires processing which depends upon what is known of the noise characteristics and of the signal. When the signal has an unknown form, it is sometimes appropriate to consider the signal as a sample function of a random process [81]. When the signal statistics are known, this knowledge can often be used to design a suitable detector. In the case of CR networks, it seems appropriate to use an energy detector to determine the activity of the primary network due to the absence of much knowledge concerning the primary user signals. The energy detector is a threshold device which measures the energy of the received waveform over an observation time window [30].

The energy detector consists of a square law device followed by a finite time integrator. First the input signal is filtered with a band-pass filter to select the bandwidth of interest. The filtered signal is then squared and integrated over a time interval, $T$. The requirement of $T$ is that it must be short in comparison to the time required for the fading amplitude 
to change up appreciably, but long in comparison to the period of the signal [76]. Fig. 2.3, depicts the block diagram of a typical energy detector.

For an arbitrary CR user, the signal receiver over the sensing link $y_{s}$ at time $t$ can be represented as

$$
\begin{array}{ll}
H_{0}: & y_{s}(t)=n_{s}(t) \\
H_{1}: & y_{s}(t)=h_{s} \cdot x_{p}(t)+n_{s}(t)
\end{array}
$$

where, $x_{p}$ denotes the signal transmitted by the primary radio, $h_{s}$ denotes the channel fading coefficient of the sensing link, and $n_{s}$ denotes the additive Gaussian noise. The hypotheses $H_{0}$ and $H_{1}$ are for the primary signal's absence and presence, respectively. For local detection, the power of $y_{s}$ denoted by $Y_{s}$ is compared with a given threshold $\lambda$ to infer the primary state following a decision rule given by [24]

$$
Y_{s} \underset{H_{0}}{\stackrel{H_{1}}{\gtrless}} \lambda
$$

By definition, a false alarm occurs when the primary user activity is claimed under $H_{0}$, while an accurate detection occurs when the primary user activity is claimed under $H_{1}$. Therefore, the detection probability, $P_{d}$, and the false alarm probability, $P_{f}$, are

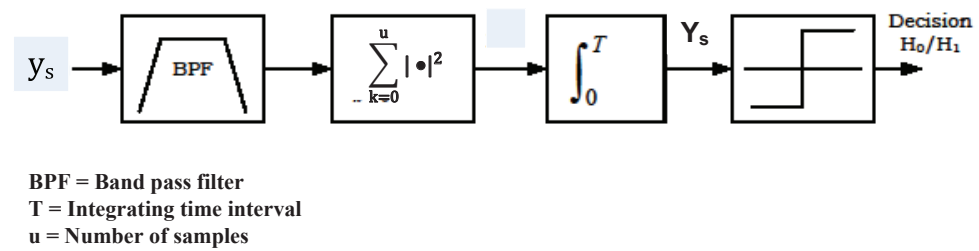

Figure 2.3: Typical energy detection receiver 
given by,

$$
\begin{aligned}
& P_{d}=P\left\{Y>\lambda \mid H_{1}\right\} \\
& P_{f}=P\left\{Y>\lambda \mid H_{0}\right\}
\end{aligned}
$$

\subsection{Cluster-Based Spectrum Sensing}

Cluster-based spectrum sensing is divided into rounds of three phases: sensing, cluster setup, and transmission phase. During the sensing period, spectrum holes are located and channel sets are made available for data exchange. CR users need to synchronize their spectrum sensing phase to avoid false alarms that may be triggered by some CRs that started their spectrum sensing earlier [89]. In the second phase, clusters are formed and cluster heads are elected. Finally, the transmission phase starts when CR terminals communicate and exchange data using the set of sensed channels that are originally owned by the primary network. The available band is only utilized during this phase and it stays idle during the sensing and clustering phases. The length of the transmission period determines how efficiently the available spectrum band can be utilized. Thus, even though the cluster set-up time is much shorter than the channel transmission time, it is not preferable to perform the cluster set-up at every sensing round so as to improve sensing efficiency. Fig. 2.4 illustrates the sensing round structure for a cluster-based cooperative sensing.

Network traffic in cluster-based systems is generated mainly by intra-cluster and inter- 


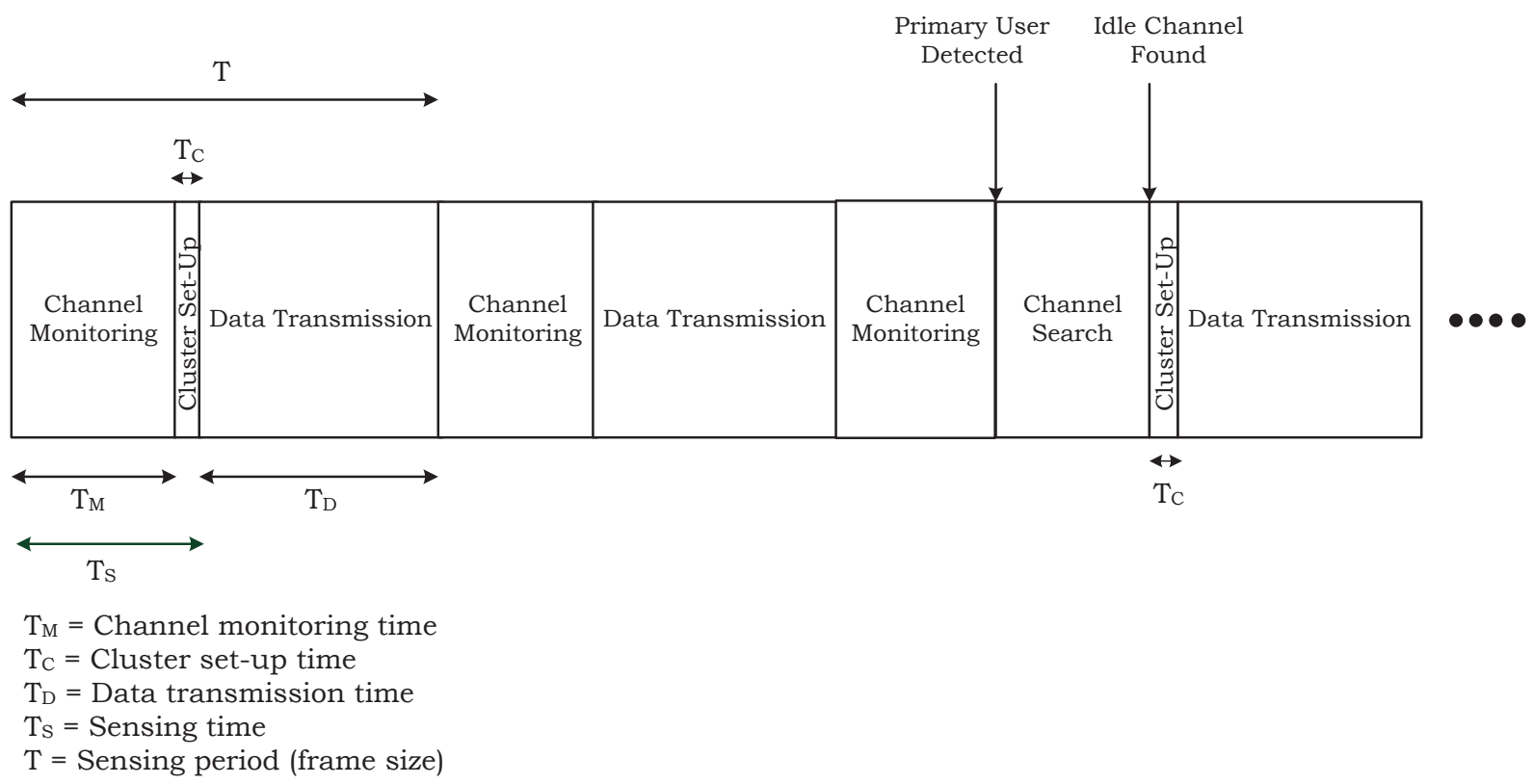

Figure 2.4: Sensing round structure in cluster-based spectrum sensing

cluster communications. Inter-cluster communications happen between cluster heads and traffic relay gateways. A gateway is a CR user who might be in one-hop from two neighboring cluster heads in case of overlapping clusters or in one-hop to another gateway in an adjacent cluster in the case of disjoint clusters. In both traffic types, the packets generated by a source node may reach the destination node through a singlehop or a multi-hop routing. For clustering to be effective, the number of cluster heads, gateways, and the links that are connecting them must be minimized while preserving the connectivity of the whole network [90]. Minimizing the number of clusters reduces the overhead traffic and the network maintenance requirements. However, clustering must consider the traffic load at both cluster heads and the gateways as these nodes tend to be the bottlenecks of the entire network. Setting up an upper bound for the cluster size is essential here as it prevents the overcrowding of nodes in the clusters and avoids 
traffic congestion at the interconnection nodes. Typical structures of the centralized and distributed CSS systems are shown in Fig. (2.5).
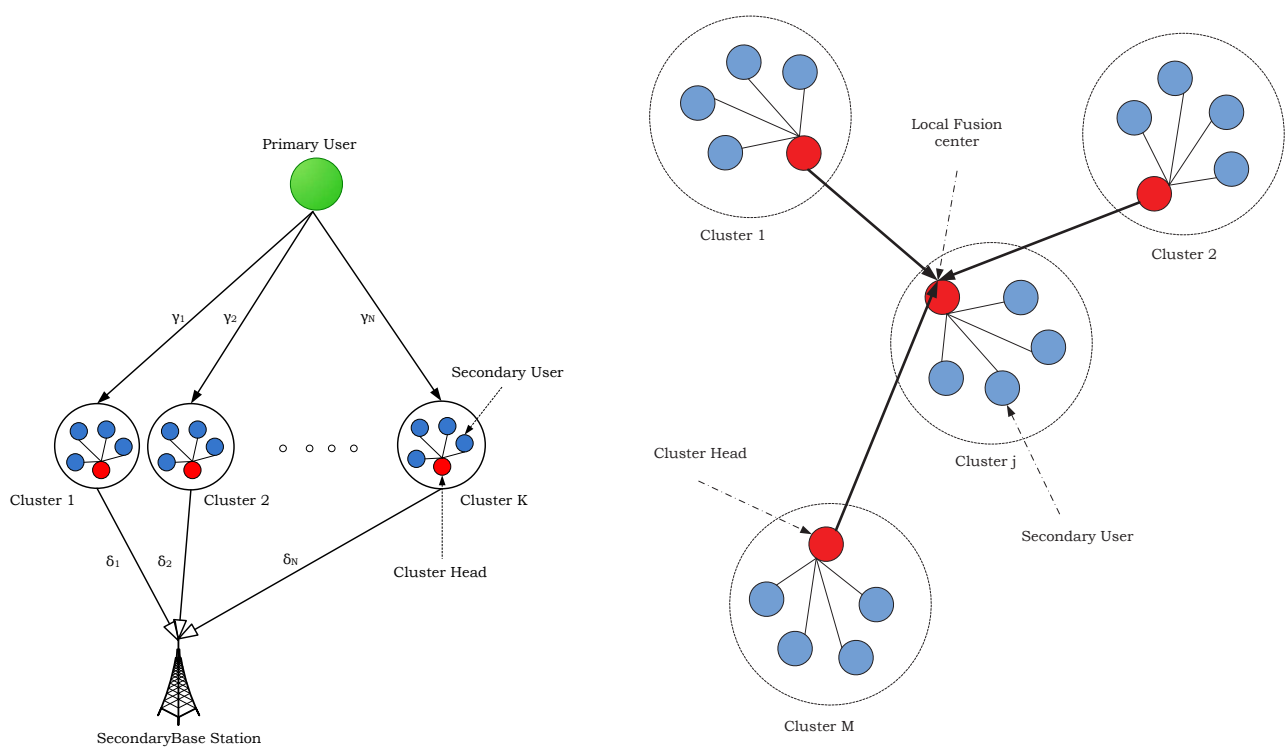

Figure 2.5: Cooperative spectrum system strategies a) centralized system b) distributed system

In this approach, the CRs are allowed to be grouped into separate clusters then each group elects a cluster head to forward a cluster decision or a cluster combined observation to a common receiver. One of the elected cluster heads is selected here to act as the common receiver i.e. a local fusion center. The common receiver decides whether the sensed band is vacant or occupied by the primary user. The final decision is then broadcasted back to the clusters. 


\section{Chapter 3}

\section{Amplify-and-Forward Strategy over}

\section{Non-Identical Fading Channels}

\subsection{Introduction}

In this chapter, we investigate the detection accuracy of an AF relay-based CSS approach over non-identical Nakagami- $m$ fading channels. We derive new exact and approximated closed-form expressions for the average detection probability and the average false alarm probability over two diversity combining techniques: EGC scheme and SC scheme. We also investigate the convergence rate of infinite series that appears in the derived exact closed-form expressions and propose to use a powerful acceleration algorithm that allows for the series termination with a finite number of terms.

Performance analysis of wireless communication networks usually involves compli- 
cated and cumbersome statistical methods. Several statistical models have been introduced in the literature to describe the fading envelope of the received signal [78, 91-96]. For short term fades, the Rayleigh distribution can be used to characterize the envelope of faded signals over small propagation area while log-normal distribution is used when much wider geographical areas are involved [78]. However, Nakagami- $m$ distribution [91] is the most versatile statistical model which can model a variety of fading environments. Nakagami distribution has a relatively simple analytical form, making it attractive in performance analysis. The fading parameter of the Nakagami- $m$ distribution can describe both severe and weak fading. Therefore, the distribution can be employed in the presence or absence of LOS between the user terminal and the base station [92, 97]. Furthermore, the Nakagami distribution is more flexible and more accurately fits the experimental data for many physical propagation channels than the Log-normal and Rician distributions [78].

The early reported results on communication over Nakagami-fading channels have been obtained based on the use of variants of the CF or the MGF of the sum of gamma variates [98-100]. However, in [101, 102], the authors proposed general approaches related to independent nonidentical distribution of gamma RVs then derived infinite-series expressions for the PDF of the sum of gamma variates for queuing type of problems. Their works have been extended in [95] where the case of arbitrary correlated gamma RVs are included in a performance study of MRC and EGC receivers.

Motivated by the above considerations, we investigate a new relay-based CSS strate- 
gies that incorporates sensing links, relaying links, diversity combining schemes, and energy detection into a general framework for a comprehensive performance analysis of CR networks with diversity reception. The proposed framework adopts the AF strategy as a relaying mechanism, using an energy detection technique and applying the soft-fusion policy to facilitate the cooperative decision.

\subsection{Spectrum Sensing Model}

A centralized CR network with $L$ active secondary users is considered. The cooperative decision is assumed to be made by a fusion center. The secondary users, $\left\{C R_{i}\right\}_{i=1}^{L}$, stand for the system relays and share the same spectrum band, which is originally allocated to the primary users. The channel fading parameters of the $i$ th sensing link and the $i$ th relaying link are denoted by $h_{s_{i}}$ and $h_{r_{i}}$, respectively. We also denote by $n_{s_{i}}$ and $n_{r_{i}}$, the additive Gaussian noise of the sensing and the relaying channels, respectively, which are modeled as white Gaussian random processes that are assumed to be i.i.d with zero mean and variance $N_{0}$.

We assume that channel state information (CSI) is available for all the relays. We also, assume that the fusion center has a full knowledge of CSI, i.e., it can acquire global knowledge of sensing and relaying channel gains. CSI is one of the main requisites for successful implementation of dynamic cognitive radio protocols. In practice, it is easy to obtain local knowledge of channel gains via a feedback mechanism by using pilot signals transmitted periodically by the fusion center $[26,103]$. In order to obtain the CSI of the 
distant link (i.e. sensing link), CR amplifies a pilot signal received from the primary base station and forwards it to the fusion center [104].

Let $x_{p}$ denotes the signal transmitted by the primary radio, then the received signal by the ith CR user, $y_{s_{i}}$, can be expressed as

$$
y_{s_{i}}=\theta h_{s_{i}} x_{p}+n_{s_{i}}
$$

where, $\theta=0$ or 1 denotes the primary user state under two hypotheses: $H_{o}$ for primary user absence and $H_{1}$ for primary user presence. If $Y_{s_{i}}$ denotes the power of $y_{s_{i}}$, then the mean value of $Y_{s_{i}}$ can be expressed as [20]

$$
\mathrm{E}\left\{Y_{s_{i}}\right\}= \begin{cases}\sigma_{Y_{s_{i}}}=N_{0} & H_{0} \\ \sigma_{Y_{s_{i}}}=E_{i}+N_{0}=N_{0}\left(1+\bar{\gamma}_{s_{i}}\right) & H_{1}\end{cases}
$$

where, $E_{i}=\mathrm{E}\left\{\left|h_{s_{i}} x_{p}\right|^{2}\right\}$ is the mean value of the signal power as received at the RF front-end of the $i$ th $C R$ receiver and $\bar{\gamma}_{s_{i}}=E_{i} / N_{0}$ is the average SNR associated with the $i$ th sensing link. For local spectrum sensing, $Y_{s_{i}}$ is compared with a given threshold $\lambda_{i}$ to infer the primary state $\theta$. Hence, the false alarm probability, $P_{f_{i}}$, and the detection probability, $P_{d_{i}}$, can be expressed as

$$
\begin{aligned}
P_{f_{i}} & =P\left\{Y_{s_{i}}>\lambda_{i} \mid H_{0}\right\}=\int_{\lambda_{i}}^{\infty}\left(\frac{m_{i}}{\sigma_{Y_{s_{i}}}}\right)^{m_{i}} \frac{y^{m_{i}-1}}{\Gamma\left(m_{i}\right)} e^{\left(-\frac{m_{i}}{\sigma_{Y_{s_{0}}}}\right) y} d y \\
& =\frac{\Gamma\left(m_{i}, \frac{m_{i} \lambda_{i}}{\sigma_{Y_{s_{i}}}}\right)}{\Gamma\left(m_{i}\right)}=\frac{\Gamma\left(m_{i}, \frac{m_{i} \lambda_{i}}{N_{0}}\right)}{\Gamma\left(m_{i}\right)}
\end{aligned}
$$


and

$$
\begin{aligned}
P_{d_{i}} & =P\left\{Y_{s_{i}}>\lambda_{i} \mid H_{1}\right\}=\int_{\lambda_{i}}^{\infty}\left(\frac{m_{i}}{\sigma_{Y_{s_{1}}}}\right)^{m_{i}} \frac{y^{m_{i}-1}}{\Gamma\left(m_{i}\right)} e^{\left(-\frac{m_{i}}{\sigma_{Y_{s_{1}}}}\right) y} d y \\
& =\frac{\Gamma\left(m_{i}, \frac{m_{i} \lambda_{i}}{\sigma_{Y_{s_{1}}}}\right)}{\Gamma\left(m_{i}\right)}=\frac{\Gamma\left(m_{i}, \frac{m_{i} \lambda_{i}}{N_{0}\left(1+\bar{\gamma}_{s_{i}}\right)}\right)}{\Gamma\left(m_{i}\right)}
\end{aligned}
$$

where, the above integrals is evaluated with the help of [105, Eq. 3.381.3], $m_{i}$ is the Nakagami- $m$ fading parameter, $\Gamma($.$) is the gamma function as defined in [106, Eq.(6.1.1)],$ and $\Gamma(.,$.$) is the incomplete Gamma function defined in [106, Eq. (6.5.3)], .$

As can be seen from the expression given in (3.3), the false alarm probability of the individual local detection is not related to the SNR and mainly depends on the decision threshold, $\lambda_{i}$. Therefore, to employ the energy detection, $\lambda_{i}$, must be properly selected to achieve a target detection accuracy. In most research works, e.g. $[13,26,27], \lambda_{i}$ is selected such that the false alarm probability is bounded by an upper target value. A value of $\bar{P}_{f}<0.1$ is recommended as an upper bound by IEEE 802.22 standard [107].

\subsubsection{Single-Relay System}

According to the AF relaying strategy, CR users are allowed to amplify the received signal and directly relay to the fusion center. We assume that every CR relay has a maximum power constraint, $P_{i}$. Hence, it measures the average received signal power and scales it appropriately so that the power constraint is satisfied. Accordingly, the signal received 
at the fusion center takes the form

$$
y_{i}=h_{r_{i}}\left(\sqrt{A_{i}}\left(\theta h_{s_{i}} x_{p}+n_{s_{i}}\right)\right)+n_{r_{i}}=\theta \sqrt{A_{i}} h_{r_{i}} h_{s_{i}} x_{p}+\sqrt{A_{i}} h_{r_{i}} n_{s_{i}}+n_{r_{i}}
$$

where, $A_{i}$ denotes the dimensionless amplification factor of the $i$ th CR relay. According to the maximum power constraint, $A_{i}$ is selected as [69]

$$
A_{i}=\frac{P_{i}}{E_{i}+N_{0}}
$$

The second term in equation (3.5) clearly indicates that the noise in the dual-hop system cannot be independent of the channel statistics. Accordingly, the propagation characteristics of the second hop must be considered when computing the false alarm probability and the detection probability.

In order to accommodate the two hypotheses $H_{0}$ and $H_{1}$, we define $g_{i}=\left|h_{r_{i}}\right|^{2}$ as the instantaneous channel gain of the $i$ th relaying link and $\bar{g}_{i} \triangleq \mathrm{E}\left\{\left|h_{r_{i}}\right|^{2}\right\}$ as the expected value of $g_{i}$. Since $h_{r_{i}}$ follows a Nakagami- $m$ distribution, it is easy to verify that $g_{i}$ follows a gamma distribution given by

$$
f_{G_{i}}(g)=\left(\frac{m_{i}}{\bar{g}_{i}}\right)^{m_{i}} \frac{g^{m_{i}-1}}{\Gamma\left(m_{i}\right)} e^{\left(-\frac{m_{i}}{\bar{g}_{i}}\right) g}, \quad g \geq 0 .
$$

Let $Y_{i}$ denotes the power of the the relayed signal $y_{i}$, then from (3.5), the mean value 
of $Y_{i}$ for a given $g$ can be expressed as

$$
\mathrm{E}\left\{Y_{i} \mid g\right\}= \begin{cases}\sigma_{Y_{i_{0}}}=N_{0}\left(1+A_{i} g\right) & H_{0} \\ \sigma_{Y_{i_{1}}}=N_{0}\left(1+\left(1+\bar{\gamma}_{s_{i}}\right) A_{i} g\right) & H_{1}\end{cases}
$$

Therefore, the probability of the false alarm for a given $g$ can be evaluated as

$$
\begin{aligned}
\bar{P}_{f_{i} \mid g}^{A F} & =P\left\{Y_{i}>\lambda \mid H_{0}, g\right\} \\
& =\int_{\lambda}^{\infty}\left(\frac{m_{i}}{\sigma_{Y_{i_{0}}}}\right)^{m_{i}} \frac{y^{m_{i}-1}}{\Gamma\left(m_{i}\right)} e^{\left(-\frac{m_{i}}{\sigma_{Y_{i}}}\right) y} d y \\
& =\frac{1}{\Gamma\left(m_{i}\right)} \Gamma\left(m_{i}, \frac{m_{i} \lambda}{N_{0}\left(1+A_{i} g\right)}\right)
\end{aligned}
$$

where, the integral in (3.9) is evaluated with the help of [105, Eq. 3.383.5] and $\lambda$ denotes the decision threshold used by the fusion center to infer the primary state $\theta$. Now, we remove the condition on $g$ and compute an average false alarm probability $\bar{P}_{f_{i}}$ by integrating over the PDF of the channel gain given in (3.7) as follows

$$
\begin{aligned}
\bar{P}_{f_{i}}^{A F} & =\int_{0}^{\infty} P\left\{Y_{i}>\lambda \mid H_{0}, g\right\} f_{G_{i}}(g) d g \\
& =\int_{0}^{\infty} \frac{1}{\Gamma\left(m_{i}\right)} \Gamma\left(m_{i}, \frac{m_{i} \lambda}{N_{0}\left(1+A_{i} g\right)}\right) f_{G_{i}}(g) d g .
\end{aligned}
$$

Evaluating the integral in (3.10) as described in Appendix A.1, $\bar{P}_{f_{i}}^{A F}$ can be expressed 
mathematically as

$$
\bar{P}_{f_{i}}^{A F}=\left(\frac{\beta_{i}}{A_{i}}\right)^{m_{i}} \sum_{q=0}^{m_{i}-1} \frac{1}{q !}\left(\frac{m_{i} \lambda}{N_{0}}\right)^{q} \sum_{n=0}^{\infty}(-1)^{n} b_{n} U\left(m_{i} ; m_{i}+1-q-n ; \frac{\beta_{i}}{A_{i}}\right)
$$

where, $\beta_{i}=m_{i} / \bar{g}_{i}, b_{n}=\frac{1}{n !}\left(\frac{m_{i} \lambda}{N_{0}}\right)^{n}$, and $U(. ; ; . ;)$ is the confluent hypergeometric function of the second kind defined in [106, Eq. 13.1.3]. Similarly, the average detection probability $\bar{P}_{d_{i}}$ can be obtained as

$$
\bar{P}_{d_{i}}^{A F}=\left(\frac{\beta_{i}}{\left(1+\bar{\gamma}_{s_{i}}\right) A_{i}}\right)^{m_{i}} \sum_{q=0}^{m_{i}-1} \frac{1}{q !}\left(\frac{m_{i} \lambda}{N_{0}}\right)^{q} \sum_{n=0}^{\infty}(-1)^{n} b_{n} U\left(m_{i} ; m_{i}+1-q-n ; \frac{\beta_{i}}{\left(1+\bar{\gamma}_{s_{i}}\right) A_{i}}\right) .
$$

\subsubsection{Multi-Relay System}

\subsubsection{Selection Combining Scheme}

In the performance analysis of cooperative diversity techniques, the statistic of the channel with maximum gain is often important [10]. The selection combining technique is often used to identify this channel among all the diversity branches. It is worth noting that SC technique can be implemented in two different strategies [108]. In the first strategy, the combiner selects the relaying branch with the highest $\mathrm{SNR}, \gamma_{r}$, while in the second strategy, the relay with the highest $\min \left(\gamma_{s}, \gamma_{r}\right)$ is selected. In our CSS model, we employ the first strategy, however, instead of the SNR, the channel gain will be used to accommodate the two hypotheses, $H_{0}$ and $H_{1}$. Therefore, for $L$ inputs, the output of the 
SC receiver is expressed as: $g_{\max }=\max \left(g_{1}, g_{2}, \cdots, g_{L}\right)$. If $Y$ denotes the signal power at the output of the combiner then the mean value of $Y$ for a given $g$ can be expressed as

$$
\mathrm{E}\{Y \mid g\}= \begin{cases}\sigma_{Y_{0}}=N_{0}\left(1+A_{S C} g\right) & H_{0} \\ \sigma_{Y_{1}}=N_{0}\left[1+\left(1+\bar{\gamma}_{s_{S C}}\right) A_{S C} g\right] & H_{1}\end{cases}
$$

where, $A_{S C} \in\left\{A_{i}\right\}_{i=1}^{L}$ and $\bar{\gamma}_{s_{S C}} \in\left\{\bar{\gamma}_{s_{i}}\right\}_{i=1}^{L}$ denote the amplification factor and the average SNR associated with the selected relay, respectively. The average false alarm probability, $\bar{P}_{f_{S C}}$ is evaluated by averaging over the PDF of $g_{\max }$ as follows

$$
\begin{aligned}
\bar{P}_{f_{S C}} & =\int_{0}^{\infty} P\left\{Y>\lambda \mid H_{0}, g\right\} f_{G_{\max }}(g) d g \\
& =\int_{0}^{\infty} \frac{1}{\Gamma\left(m_{S C}\right)} \Gamma\left(m_{S C}, \frac{m_{S C} \lambda}{N_{0}\left(1+A_{S C} g\right)}\right) f_{G_{\max }}(g) d g
\end{aligned}
$$

where, $m_{S C} \in\left\{m_{i}\right\}_{i=1}^{L}$ denotes the Nakagami- $m$ fading parameter of the link associated with the selected relay. With the help of Appendix A.2, $\bar{P}_{f_{S C}}$ can be expressed mathematically as follows

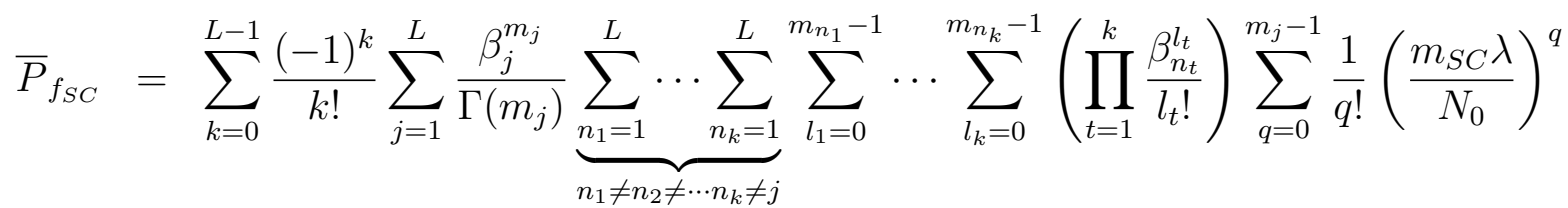

$$
\begin{aligned}
& \frac{\Gamma\left(\nu_{k j}\right)}{A_{S C}^{\nu_{k j}}} \sum_{n=0}^{\infty}(-1)^{n} b_{n} U\left(\nu_{k j} ; \nu_{k j}+1-q-n ; \frac{\mu_{k j}}{A_{S C}}\right)
\end{aligned}
$$


where, $b_{n}=\frac{1}{n !}\left(\frac{m_{S C} \lambda}{N_{0}}\right)^{n}, \nu_{k j}=\sum_{t=1}^{k} l_{t}+m_{j}$, and $\mu_{k j}=\sum_{t=1}^{k} \beta_{n_{t}}+\beta_{j}$. Similarly

$$
\begin{aligned}
& \bar{P}_{d_{S C}}=\sum_{k=0}^{L-1} \frac{(-1)^{k}}{k !} \sum_{j=1}^{L} \frac{\beta_{j}^{m_{j}}}{\Gamma\left(m_{j}\right)} \underbrace{\sum_{n_{1}=1}^{L} \cdots \sum_{n_{k}=1}^{L}}_{n_{1} \neq n_{2} \neq \cdots n_{k} \neq j} \sum_{l_{1}=0}^{m_{n_{1}}-1} \cdots \sum_{l_{k}=0}^{m_{n_{k}}-1}\left(\prod_{t=1}^{k} \frac{\beta_{n_{t}}^{l_{t}}}{l_{t} !}\right)^{m_{j}-1} \frac{1}{q=0}\left(\frac{m_{S C} \lambda}{N_{0}}\right)^{q} \\
& \frac{\Gamma\left(\nu_{k j}\right)}{\left(\left(1+\bar{\gamma}_{s_{S C}}\right) A_{S C}\right)^{\nu_{k j}}} \sum_{n=0}^{\infty}(-1)^{n} b_{n} U\left(\nu_{k j} ; \nu_{k j}+1-q-n ; \frac{\mu_{k j}}{\left(1+\bar{\gamma}_{s_{S C}}\right) A_{S C}}\right)
\end{aligned}
$$

As a by-product of the above result, $\bar{P}_{f_{S C}}$ and $\bar{P}_{d_{S C}}$ over Rayleigh channels, $\bar{P}_{f_{S C_{R a y}}}$ and $\bar{P}_{d_{S C_{R a y}}}$, respectively, can be obtained by setting $m_{j}=1$ and $m_{S C}=1$ in (3.15) and $(3.16)^{2}$

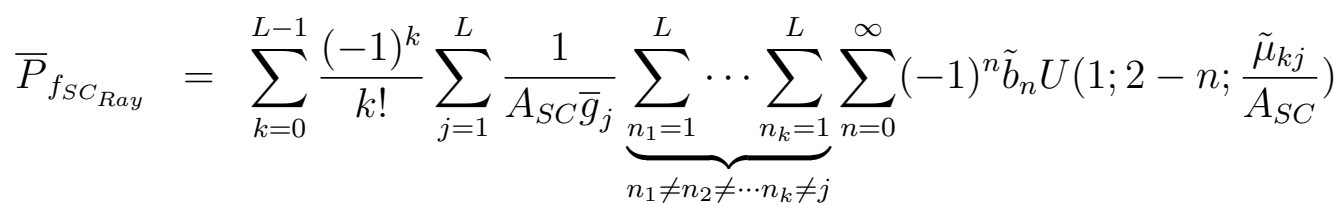

and

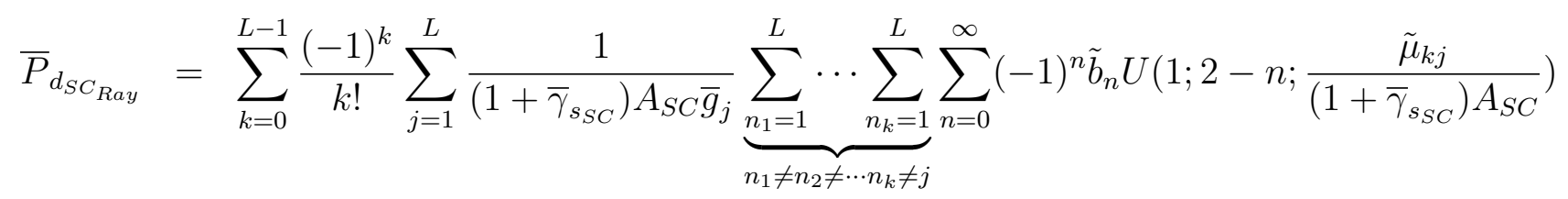

where, $\tilde{b}_{n}=\frac{1}{n !}\left(\frac{\lambda}{N_{0}}\right)^{n}$ and $\tilde{\mu}_{k j}=\sum_{t=1}^{k}\left(\frac{1}{\bar{g}_{n_{t}}}\right)+\left(\frac{1}{\bar{g}_{j}}\right)$.

\footnotetext{
${ }^{2} m=1$ is a special case of Nakagami- $m$ fading referred to be as a Rayleigh fading
} 
Over i.i.d Nakagami- $m$ channels $\bar{P}_{f_{S C}}$ and $\bar{P}_{d_{S C}}$ are expressed as follows

$$
\begin{aligned}
\bar{P}_{f_{S C}}= & \frac{\beta^{m}}{\Gamma(m)} \sum_{k=0}^{L-1} \frac{(-1)^{k}}{k !} \sum_{j=1}^{L} \underbrace{\sum_{n_{1}=1}^{L} \cdots \sum_{n_{k}=1}^{L}}_{n_{1} \neq n_{2} \neq \cdots n_{k} \neq j} \sum_{l_{1}=0}^{m-1} \cdots \sum_{l_{k}=0}^{m-1}\left(\prod_{t=1}^{k} \frac{\beta^{l_{t}}}{l_{t} !}\right) \sum_{q=0}^{m-1} \frac{1}{q !}\left(\frac{m \lambda}{N_{0}}\right)^{q} \\
& \frac{\Gamma\left(\nu_{k}\right)}{A^{\nu_{k}}} \sum_{n=0}^{\infty}(-1)^{n} b_{n} U\left(\nu_{k} ; \nu_{k}+1-q-n ; \frac{\mu_{k}}{A}\right)
\end{aligned}
$$

and

$$
\begin{aligned}
& \bar{P}_{d_{S C}}=\frac{\beta^{m}}{\Gamma(m)} \sum_{k=0}^{L-1} \frac{(-1)^{k}}{k !} \sum_{j=1}^{L} \underbrace{\sum_{n_{1}=1}^{L} \cdots \sum_{n_{k}=1}^{L}}_{n_{1} \neq n_{2} \neq \cdots n_{k} \neq j} \sum_{l_{1}=0}^{m-1} \cdots \sum_{l_{k}=0}^{m-1}\left(\prod_{t=1}^{k} \frac{\beta^{l_{t}}}{l_{t} !}\right) \sum_{q=0}^{m-1} \frac{1}{q !}\left(\frac{m \lambda}{N_{0}}\right)^{q} \\
& \frac{\Gamma\left(\nu_{k}\right)}{\left(\left(1+\bar{\gamma}_{s}\right) A\right)^{\nu_{k}}} \sum_{n=0}^{\infty}(-1)^{n} b_{n} U\left(\nu_{k} ; \nu_{k}+1-q-n ; \frac{\mu_{k}}{\left(1+\bar{\gamma}_{s}\right) A}\right) \text {. }
\end{aligned}
$$

In this case, it is important to mention that $\nu_{k}=\sum_{t=1}^{k} l_{t}+m$ and $\mu_{k}=(k+1) \beta$.

\subsubsection{Equal Gain Combining Scheme}

Many performance analysis problems require determination of statistics of the sum of the squared envelopes of the faded signals over several diversity paths which can be achieved through EGC technique. For $L$ inputs, the output of the EGC receiver can be expressed as

$$
y=\sum_{i=1}^{L} \sqrt{A_{i}} h_{r_{i}}\left(\theta h_{s_{i}} x_{p}+n_{s_{i}}\right)+n_{r_{i}}=\theta \sum_{i=1}^{L} \sqrt{A_{i}} h_{r_{i}} h_{s_{i}} x_{P}+\sum_{i=1}^{L} \sqrt{A_{i}} h_{r_{i}} n_{s_{i}}+n_{r_{i}} .
$$

If $Y$ denotes the power at the output of the EGC receiver, then for given $g_{i}$ 's, the 
mean value of $Y$ can be expressed as

$$
\mathrm{E}\left\{Y \mid g_{i}{ }^{\prime} s\right\}= \begin{cases}\sigma_{Y_{0}}=N_{0}\left(1+\sum_{i=1}^{L} A_{i} g_{i}\right) & H_{0} \\ \sigma_{Y_{1}}=N_{0}\left(1+\sum_{i=1}^{L}\left(1+\bar{\gamma}_{s_{i}}\right) A_{i} g_{i}\right) & H_{1}\end{cases}
$$

Therefore, the average false alarm probability for the EGC case is given by

$$
\begin{aligned}
\bar{P}_{f_{E G C}} & =P\left\{Y \geq \lambda \mid H_{0}\right\}=\int_{0}^{\infty} \cdots \int_{0}^{\infty} P\left\{Y \geq \lambda \mid H_{0}, g_{1}, \cdots g_{L}\right\} \cdot f\left(g_{1} \mid H_{0}\right) \cdots f\left(g_{L} \mid H_{0}\right) d g_{1} \cdots d g_{L} \\
& =\int_{0}^{\infty} \cdots \int_{0}^{\infty} \frac{1}{\Gamma\left(m_{j}\right)} \Gamma\left(m_{j}, \frac{m_{j} \lambda}{N_{0}\left(1+\sum_{i=1}^{L} A_{i} g_{i}\right)}\right) \cdot f\left(g_{1} \mid H_{0}\right) \cdots f\left(g_{L} \mid H_{0}\right) d g_{1} \cdots d g_{L} .
\end{aligned}
$$

The expression of $\bar{P}_{f_{E G C}}$ in (4.16) cannot be evaluated straightforward. However, we can rewrite this expression as the expectation over $g_{i}$ 's such that

$$
\bar{P}_{f_{E G C}}=\mathrm{E}_{g_{1}, \cdots, g_{L}}\left\{P\left\{Y \geq \lambda \mid H_{0}, g_{1}, \cdots g_{L}\right\}\right\}=\mathrm{E}_{g_{1}, \cdots, g_{L}}\left\{\frac{1}{\Gamma\left(m_{j}\right)} \Gamma\left(m_{j}, \frac{m_{j} \lambda}{N_{0}\left(1+\sum_{i=1}^{L} A_{i} g_{i}\right)}\right)\right\} .
$$

To simplify the computations of the above expectation, we define the following random variable

$$
R=\sum_{i=1}^{L} A_{i} g_{i}
$$

Since each $g_{i}$ follows gamma distribution, the random variable, $R$, is a sum of mutually independent gamma variates. Thus the expression of $\bar{P}_{f_{E G C}}$ in (3.24) can be expressed 
as

$$
\bar{P}_{f_{E G C}}=\int_{0}^{\infty} \frac{1}{\Gamma\left(m_{j}\right)} \Gamma\left(m_{j}, \frac{m_{j} \lambda}{N_{0}(1+r)}\right) f_{R}(r) d r
$$

where, $f_{R}(r)$ is the PDF of the random variable $R$. The above integral can be evaluated as described in Appendix A.3, yielding

$\bar{P}_{f_{E G C}}=\left[\prod_{j=1}^{L}\left(-\frac{\beta_{j}}{A_{j}}\right)^{m_{j}}\right] \sum_{j=1}^{L} \sum_{v=1}^{m_{j}}(-1)^{v} b_{j v} \sum_{q=0}^{m_{j}-1} \frac{1}{q !}\left(\frac{m_{j} \lambda}{N_{0}}\right)^{q} \sum_{n=0}^{\infty}(-1)^{n} b_{n} U\left(v ; v+1-q-n ; \frac{\beta_{j}}{A_{j}}\right)$

Similarly, the average detection probability can be expressed as

$$
\begin{aligned}
\bar{P}_{d_{E G C}}= & {\left[\prod_{j=1}^{L}\left(-\frac{\beta_{j}}{\left(1+\bar{\gamma}_{s_{j}}\right) A_{j}}\right)^{m_{j}}\right] \sum_{j=1}^{L} \sum_{v=1}^{m_{j}}(-1)^{v} b_{j v} \sum_{q=0}^{m_{j}-1} \frac{1}{q !}\left(\frac{m_{j} \lambda}{N_{0}}\right)^{q} } \\
& \sum_{n=0}^{\infty}(-1)^{n} b_{n} U\left(v ; v+1-q-n ; \frac{\beta_{j}}{\left(1+\bar{\gamma}_{s_{j}}\right) A_{j}}\right)
\end{aligned}
$$

where

$b_{j v}=\left[\sum_{j_{1}=0}^{m_{j}-v-1}\left(\begin{array}{c}m_{j}-v-1 \\ j_{1}\end{array}\right) B_{j}^{m_{j}-v-1-j_{1}} \sum_{j_{2=0}}^{j_{1}-1}\left(\begin{array}{c}j_{1}-1 \\ j_{1}\end{array}\right) B_{j}^{j_{1}-1-j_{2}} \sum_{j_{3=0}}^{j_{2}-1}\left(\begin{array}{c}j_{2}-1 \\ j_{1}\end{array}\right) B_{j}^{j_{2}-1-j_{3}} \cdots\right] \frac{\Delta_{j}}{\left(m_{j}-v\right) !}$

where

$$
\Delta_{j}=\underbrace{\prod_{i=1}^{L}}_{i \neq j}\left(\frac{\beta_{j}}{A_{j}}-\frac{\beta_{i}}{A_{i}}\right)^{m_{i}}
$$


and

$$
B_{j}^{t}=(-1)^{t+1} t ! \underbrace{\sum_{i=1}^{L}}_{i \neq j} m_{j}\left(\frac{\beta_{j}}{A_{j}}-\frac{\beta_{i}}{A_{i}}\right)^{-(t+1)}
$$

Over Rayleigh fading channels, $\bar{P}_{f_{E G C_{R a y}}}$ and $\bar{P}_{d_{E G C_{R a y}}}$ are evaluated by setting $m_{j}=1$ in (3.27) and (3.28)

$$
\bar{P}_{f_{E G C_{R a y}}}=(-1)^{L+1}\left[\prod_{j=1}^{L} \frac{1}{A_{j} \bar{g}_{j}}\right] \sum_{j=1}^{L} \tilde{\Delta}_{j} \sum_{n=0}^{\infty}(-1)^{n} \tilde{b}_{n} U\left(1 ; 2-n ; \frac{1}{A_{j} \bar{g}_{j}}\right)
$$

and

$$
\bar{P}_{d_{E G C_{R a y}}}=(-1)^{L+1}\left[\prod_{j=1}^{L} \frac{1}{\left(1+\bar{\gamma}_{s_{j}}\right) A_{j} \bar{g}_{j}}\right] \sum_{j=1}^{L} \tilde{\Delta}_{j} \sum_{n=0}^{\infty}(-1)^{n} \tilde{b}_{n} U\left(1 ; 2-n ; \frac{1}{\left(1+\bar{\gamma}_{s_{j}}\right) A_{j} \bar{g}_{j}}\right)
$$

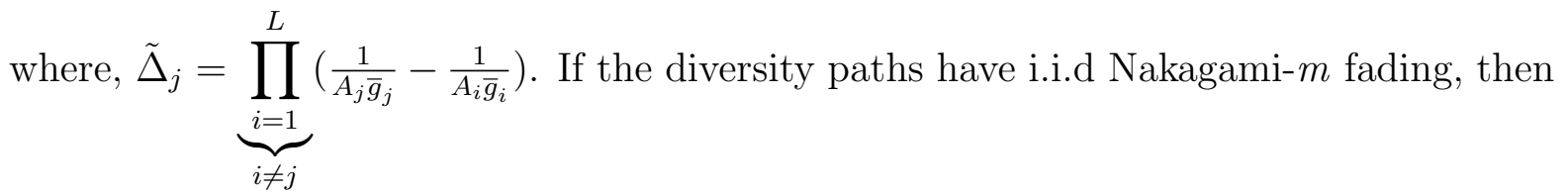
the density function of $R$ is a gamma density given by

$$
f_{R}(r)=\frac{\beta^{L m}}{A^{L m} \Gamma(L m)} r^{L m-1} e^{-\frac{\beta}{A} r}
$$

Therefore, the average false alarm probability $\bar{P}_{f}$ and the average detection probability $\bar{P}_{d}$ can be expressed as

$$
\bar{P}_{f_{E G C}}=\frac{\beta^{L m}}{A^{L m}} \sum_{q=0}^{m-1} \frac{1}{q !}\left(\frac{m \lambda}{N_{0}}\right)^{q} \sum_{n=0}^{\infty}(-1)^{n} b_{n} U\left(L m ; L m+1-q-n ; \frac{\beta}{A}\right)
$$




$$
\bar{P}_{d_{E G C}}=\frac{\beta^{L m}}{\left(\left(1+\bar{\gamma}_{s}\right) A\right)^{L m}} \sum_{q=0}^{m-1} \frac{1}{q !}\left(\frac{m \lambda}{N_{0}}\right)^{q} \sum_{n=0}^{\infty}(-1)^{n} b_{n} U\left(L m ; L m+1-q-n ; \frac{\beta}{\left(1+\bar{\gamma}_{s}\right) A}\right) .
$$

\subsubsection{Convergence Acceleration}

Theoretically, the infinite series $\sum_{n=0}^{\infty}(-1)^{n} b_{n}$ that appears in the exact closed-form expressions converges as $n \rightarrow \infty$. We observed that this series converges for relatively small values of $n$ when $\lambda$ is small and, therefore, the infinite-series expressions can be accurately computed. However, when $\lambda$ becomes large $(\lambda \rightarrow \infty), \bar{P}_{d}$ as well as $\bar{P}_{f}$ tend to be very small (for instance, their values can be less than $10^{-3}$ ). Hence, to accurately compute $\bar{P}_{d}$ and $\bar{P}_{f}$, a large number of terms need to be evaluated. Specifically, the sum of the infinite series requires the evaluation of $b_{n}$ over a large value of $n$. This shortcoming can be avoided by using a convergence acceleration technique. Series acceleration is used to improve the rate of convergence of infinite-series through sequence transformation algorithms [109]. The technique has the potential to reach the series limit within some accuracy using fewer terms than are required. Sequence transformation generates a new sequence based on a partial sum $S_{n}=\sum_{k=0}^{n}(-1)^{k} b_{k}$ for $n=0,1, \cdots, N$. The objective is to estimate $\lim _{n \rightarrow \infty} S_{n}$ by using as few partial sums as possible.

One of the powerful algorithms suitable for this purpose is Wynn's $\varepsilon$-algorithm [110] which is a simple recursive scheme that builds a triangle array called epsilon array in which each term of the sequence is determined by the three previous terms with the 
initial condition $\varepsilon_{-1}^{n}=0, \varepsilon_{0}^{n}=S_{n}$

$$
\varepsilon_{l+1}^{n}=\varepsilon_{l-1}^{n+1}+\frac{1}{\varepsilon_{l}^{n+1}-\varepsilon_{l}^{n}}, \quad l=0,1,2, \cdots
$$

The algorithm repeatedly applies the recursive expression in (3.36) to estimate the converging point of $n$ while constantly adding adequate terms of $\sum_{n=0}^{\infty}(-1)^{n} b_{n}$ to reach the required accuracy. The sequence $\left(\varepsilon_{l}^{n}\right)$ is called the $l$ th column, and its construction can be graphically represented as shown in Fig 3.1. The even columns converge faster to the limit. In particular, the upsweeping diagonals converge very quickly to the limit. This diagonal sequence (circled on Fig. 3.1) is the result of the $\varepsilon$-algorithm and is called the accelerated sequence.

\subsubsection{Approximated Analysis}

Further to the acceleration algorithm, we propose new computational approach to derive the average false alarm and the average detection probabilities. If $A_{i}$ is assumed to be high enough, an approximated expansion of the incomplete gamma function can be used as follows: for a large $A_{i}$, we have $\left(1+\bar{\gamma}_{s_{i}}\right) A_{i} g_{i} \gg 1$ and $\Gamma\left(m_{i}, \frac{m_{i}}{N_{0}\left(1+\left(1+\bar{\gamma}_{s_{i}}\right) A_{i} g_{i}\right)}\right)$ is approximated to $\Gamma\left(m_{i}, \frac{m_{i}}{N_{0}\left(1+\bar{\gamma}_{s_{i}}\right) A_{i} g_{i}}\right)$, which can be expanded as $\left(m_{i}-1\right) ! e^{\frac{m_{i}}{N_{0}\left(1+\bar{\gamma}_{s_{i}}\right) A_{i} g_{i}}}$. To show how this approximation alleviates the complexity of using the PDF-approach in deriving $\bar{P}_{f}$ and $\bar{P}_{d}$, we first derive an approximated close-form expression for $\bar{P}_{d_{S C}}$. 


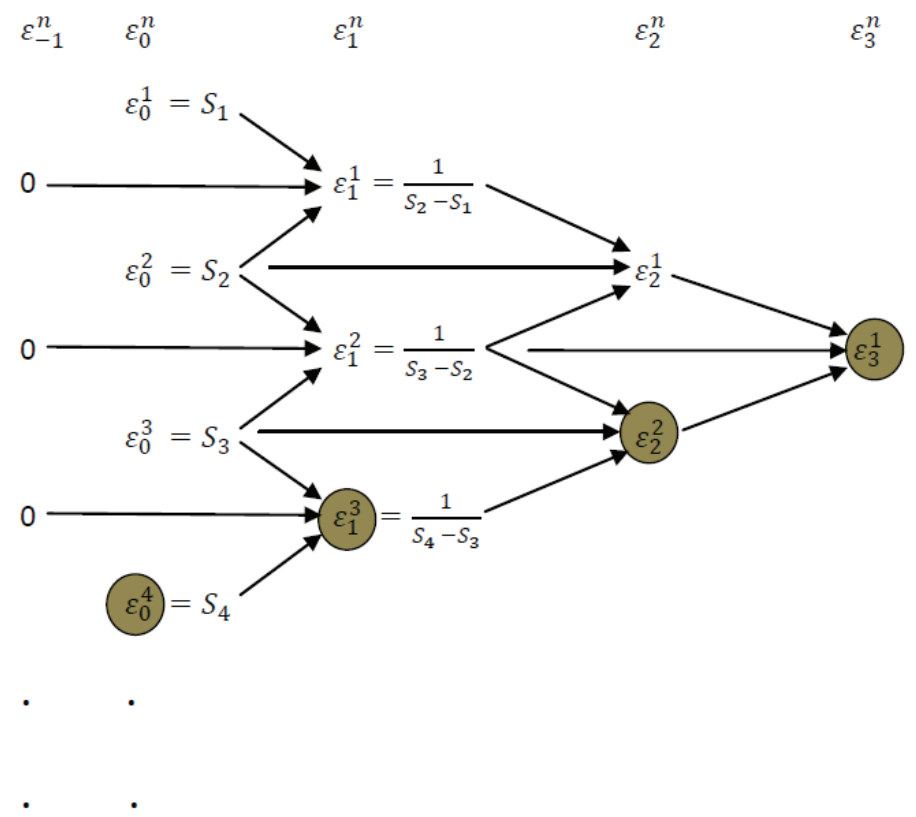

Figure 3.1: Computation structure of Wynn's $\varepsilon$-algorithm

Since the average detection probability is evaluated by averaging over the PDF of $g_{\max }$, for $\left(1+\bar{\gamma}_{S C}\right) A_{S C} g>>1$, we then get

$$
\bar{P}_{d_{S C}}=\frac{1}{\Gamma\left(m_{S C}\right)} \int_{0}^{\infty} \Gamma\left(m_{S C}, \frac{m_{S C} \lambda}{N_{0}\left(1+\bar{\gamma}_{S C}\right) A_{S C} g}\right) f_{G_{m a x}}(g) d g
$$

Substituting (A.5) for $f_{G_{\max }}(g)$ and using the series summation $\Gamma(m, x)=(m-$ $1) ! e^{-x} \sum_{n=0}^{m-1} \frac{x^{n}}{n !},(3.37)$ becomes

$$
\begin{aligned}
\bar{P}_{d_{S C}}= & \sum_{k=0}^{L-1} \frac{(-1)^{k}}{k !} \sum_{j=1}^{L} \frac{\beta_{j}^{m_{j}}}{\Gamma\left(m_{j}\right)} \underbrace{\sum_{n_{1}=1}^{L} \cdots \sum_{n_{k}=1}^{L}}_{n_{n_{1} \neq n_{2} \neq \cdots n_{k} \neq j}} \sum_{l_{1}=0}^{m_{n_{1}}-1} \cdots \sum_{l_{k}=0}^{m_{n_{k}}-1}\left(\prod_{t=1}^{k} \frac{\beta_{n_{t}}^{l_{t}}}{l_{t} !} \sum_{q=0}^{m_{i}-1} \frac{1}{q !}\left(\frac{m_{S C} \lambda}{N_{0}\left(1+\bar{\gamma}_{s_{S C}}\right) A_{S C}}\right)^{q}\right. \\
& \int_{0}^{\infty} g^{\nu_{k j}-q-1} e^{-\frac{m_{S C} \lambda}{N_{0}\left(1+\bar{\gamma}_{S C}\right) A_{S C} g}-\mu_{k j} g} d g .
\end{aligned}
$$


Using $\int_{0}^{\infty} x^{v-1} e^{-\frac{\beta}{x}-\gamma x} d x=2\left(\frac{\beta}{\gamma}\right)^{\frac{v}{2}} K_{v}(2 \sqrt{\beta \gamma})$ [105, Eq. 3.471.9], to evaluate the integral in (3.38), yields

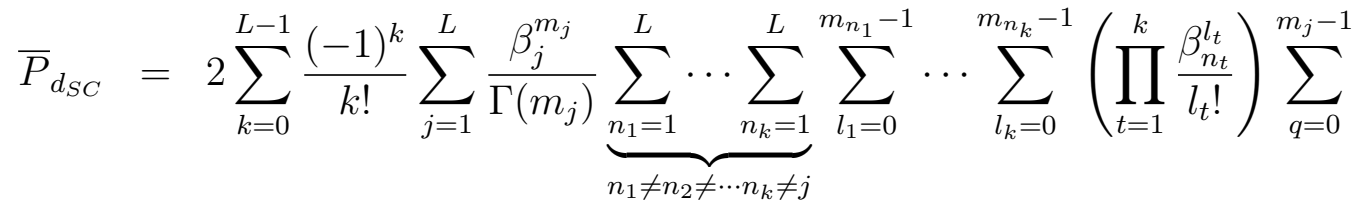

$$
\begin{aligned}
& \frac{1}{q !}\left(\frac{m_{S C} \lambda}{N_{0}\left(1+\bar{\gamma}_{s_{S C}}\right) A_{S C}}\right)^{\frac{\nu_{k j}+q}{2}}\left(\frac{1}{\mu_{k j}}\right)^{\frac{\nu_{k j}-q}{2}} K_{\nu_{k j}-q}\left(2 \sqrt{\frac{m_{S C} \lambda \mu_{k j}}{N_{0}\left(1+\bar{\gamma}_{s_{S C}}\right) A_{S C}}}\right)
\end{aligned}
$$

where, $K_{v}($.$) is the v$-order modified Bessel function of the second kind defined in [106, Eq. 9.6.22]. It is easily shown that the above expression does not have the infinite-series term, and therefore, it provides a simple computational method to compute the average detection provability.

For independent not identically distributed Rayleigh paths, we obtain

$$
\bar{P}_{d_{S C_{R a y}}}=2 \sum_{k=0}^{L-1} \frac{(-1)^{k}}{k !} \sum_{j=1}^{L} \frac{1}{\bar{g}_{j}} \underbrace{\sum_{n_{1}=1}^{L} \cdots \sum_{n_{k}=1}^{L}}_{n_{1} \neq n_{2} \neq \cdots n_{k} \neq j} \sqrt{\frac{\lambda}{N_{0}\left(1+\bar{\gamma}_{s_{S C}}\right) A_{S C} \tilde{\mu}_{k j}}} K_{1}\left(2 \sqrt{\frac{\lambda \tilde{\mu}_{k j}}{N_{0}\left(1+\bar{\gamma}_{s_{S C}}\right) A_{S C}}}\right)
$$


and for, i.i.d. Nakagami-m paths

$$
\begin{aligned}
\bar{P}_{d_{S C}}= & \frac{2 \beta^{m}}{\Gamma(m)} \sum_{k=0}^{L-1} \frac{(-1)^{k}}{k !} \sum_{j=1}^{L} \underbrace{L}_{n_{1} \neq n_{2} \neq \cdots n_{k} \neq j} \cdots \sum_{n_{k}=1}^{L} \sum_{l_{1}=0}^{m-1} \cdots \sum_{l_{k}=0}^{m-1}\left(\prod_{t=1}^{k} \frac{\beta^{l_{t}}}{l_{t} !}\right) \\
& \sum_{q=0}^{m-1} \frac{1}{q !}\left(\frac{m \lambda}{N_{0}\left(1+\bar{\gamma}_{s}\right) A}\right)^{\frac{\nu_{k}+q}{2}}\left(\frac{1}{\mu_{k}}\right)^{\frac{\nu_{k}-q}{2}} K_{\nu_{k}-q}\left(2 \sqrt{\frac{m \lambda \mu_{k}}{N_{0}\left(1+\bar{\gamma}_{s}\right) A}}\right)
\end{aligned}
$$

The approximation can be used in a similar way to derive the average detection probability of the EGC scheme. Therefore, under independent not identically distributed Nakagami- $m$ paths we have

$$
\begin{aligned}
\bar{P}_{d_{E G C}}= & 2\left[\prod_{j=1}^{L}\left(-\frac{\beta_{j}}{\left(1+\bar{\gamma}_{s_{j}}\right) A_{j}}\right)^{m_{j}}\right] \sum_{j=1}^{L} \sum_{v=1}^{m_{j}} \frac{(-1)^{v} b_{j v}}{\Gamma(v)} \sum_{q=0}^{m_{j}-1} \frac{1}{q !}\left(\frac{m_{j} \lambda}{N_{0}}\right)^{\frac{v+q}{2}}\left(\frac{\left(1+\bar{\gamma}_{s_{j}}\right) A_{j}}{\beta_{j}}\right)^{\frac{v-q}{2}} \\
& K_{v-q}\left(2 \sqrt{\frac{m_{j} \beta_{j} \lambda}{N_{0}\left(1+\bar{\gamma}_{s_{j}}\right) A_{j}}}\right) .
\end{aligned}
$$

and

$$
\begin{aligned}
\bar{P}_{d_{E G C_{R a y}}=} & 2(-1)^{L+1}\left[\prod_{j=1}^{L}\left(-\frac{1}{\left(1+\bar{\gamma}_{s_{j}}\right) A_{j} \bar{g}_{j}}\right)\right] \sum_{j=1}^{L} b_{j 1} \sqrt{\frac{\lambda\left(1+\bar{\gamma}_{s_{j}}\right) A_{j} \bar{g}_{j}}{N_{0}}} \\
& K_{1}\left(2 \sqrt{\frac{\lambda}{N_{0}\left(1+\bar{\gamma}_{s_{j}}\right) A_{j} \bar{g}_{j}}}\right) .
\end{aligned}
$$

also, for i.i.d. Nakagami-m paths, we have

$$
\bar{P}_{d_{E G C}}=\frac{2 \beta^{L m}}{\left(\left(1+\bar{\gamma}_{s}\right) A\right)^{L m} \Gamma(L m)} \sum_{q=0}^{m-1} \frac{1}{q !}\left(\frac{m \lambda}{N_{0}}\right)^{\frac{L m+q}{2}}\left(\frac{\left(1+\bar{\gamma}_{s}\right) A}{\beta}\right)^{\frac{L m-q}{2}} K_{L m-q}\left(2 \sqrt{\frac{m \beta \lambda}{N_{0}\left(1+\bar{\gamma}_{s}\right) A}}\right)
$$


The same approximation approach can also be used to derive the approximated $\bar{P}_{f_{S C}}$ and $\bar{P}_{f_{E G C}}$ under the assumption $A_{i} g_{i} \gg 1$.

To validate the accuracy of the approximation, we define

$$
I_{k}=\sum_{q=0}^{m-1} \frac{1}{q !}\left(\frac{m \lambda}{N_{0}}\right)^{q} \int_{0}^{\infty}\left(1+\left(1+\bar{\gamma}_{s}\right) A g\right)^{-q} g^{v-1} e^{-\frac{m \lambda}{N_{0}\left(1+\left(1+\bar{\gamma}_{s}\right) A g\right)}} e^{-\mu g} d g .
$$

For $\left(1+\bar{\gamma}_{s}\right) A g>>1, I_{k}$ in $(3.45)$ can be approximated as

$$
I_{k} \approx \sum_{q=0}^{m-1} \frac{1}{q !}\left(\frac{m \lambda}{N_{0}\left(1+\bar{\gamma}_{s}\right) A}\right)^{q} \int_{0}^{\infty} g^{v-q-1} e^{-\frac{m \lambda}{N_{0}\left(1+\bar{\gamma}_{s}\right) A g}} e^{-\mu g} d g .
$$

Using [105, Eq. 3.471.9], the above integral is evaluated as follows

$$
I_{k} \approx 2\left(\frac{m \lambda}{N_{0}\left(1+\bar{\gamma}_{s}\right) A}\right)^{\frac{v+q}{2}}\left(\frac{1}{\mu}\right)^{\frac{v-q}{2}} K_{v-q}\left(2 \sqrt{\frac{m \lambda \mu}{N_{0}\left(1+\bar{\gamma}_{s}\right) A}}\right)
$$

It can be seen from the closed-form expressions derived for $\bar{P}_{f}$ and $\bar{P}_{d}$ in (3.15), (3.16), (3.27), and (3.27) that all the finite sums can be calculated exactly and $I_{k}$ is the only approximated term in these expressions. Therefore, validating the computation accuracy of $I_{k}$, inevitably validates the accuracy of the expressions given in (3.39)-(4.26). In fact, validating $I_{k}$ offers a simple mean to avoid performing multiple simulation tests to validate each of the derived approximated expressions individually. In section III, the accuracy of this approximation is numerically verified by simulation results generated through the Monte Carlo test. 


\subsection{Performance Evaluation}

To validate the accuracy of the derived closed-form expressions, we assume that $L$ secondary users are deployed in a centralized CR network. The sensing and the relaying channels are assumed to be subjected to independent not identically distributed Nakagami- $m$ fading. An upper bound of $\bar{P}_{f}<0.1$ is considered for the selection of decision threshold. This upper bound is recommended in the literature as well as IEEE 802.22 standard [107]. The noise variance $N_{0}$ is set to a unity $(0 \mathrm{~dB})$.

To determine the number of terms $N$ required to evaluate the infinite-series expressions derived for $\bar{P}_{d}$ in (3.16) and (3.28), we list in table 3.1 the minimum number of terms required to evaluate these expressions for the given values of the threshold decision, $\lambda$. As can be seen, the infinite series converges with fewer number of terms for small $\lambda$ and an accuracy up to four decimal points is obtained. However, as $\lambda$ increases, the infinite series diverges and unbounded values of the average detection probability are observed. Clearly, for large $\lambda$ a large number of sum up terms is required to evaluate $\bar{P}_{d}$ using the exact expressions in (3.16) and (3.28) and it is more appropriate to use the approximated expressions in (3.39) and (3.42) due to their computational simplicity.

Table 3.1: Converging point required to evaluate $\bar{P}_{d}$ for SC and EGC schemes. $L=3$, $m=3$

\begin{tabular}{|c|c|c|c|c|c|}
\cline { 2 - 6 } \multicolumn{1}{c|}{} & \multicolumn{5}{c|}{ Converging Point $(\mathrm{n})$} \\
\cline { 2 - 6 } \multicolumn{1}{c|}{} & $\lambda=20$ & $\lambda=40$ & $\lambda=60$ & $\lambda=80$ & $\lambda=100$ \\
\hline SC Scheme, Equation(3.16) & 9 & 23 & 42 & 69 & 102 \\
\hline EGC Scheme, Equation(3.28) & 5 & 16 & 32 & 50 & 69 \\
\hline
\end{tabular}


Using a large number of terms to evaluate the infinite series, $\sum_{n=0}^{\infty}(-1)^{n} b_{n}$, significantly slows down the convergence rate. Therefore, when $\lambda$ becomes large, it is necessary to use the $\varepsilon$-algorithm to improve the convergence rate. Table 3.2 illustrates the number of terms required to evaluate $\bar{P}_{d}$ with an accuracy of up to four decimal points using the $\varepsilon$-algorithm to accelerate (3.16) and (3.28). We observed that the use of $\varepsilon$ - algorithm dramatically reduces the points of convergence $N$ and highly improves the convergence rate.

To validate the accuracy of the approximated expression, we plot in Fig. $3.2, I_{k}$ versus $\lambda$ for $P=0,5,10$, and $20 \mathrm{~dB}$. In this figure, the analytical results obtained from the $I_{k}$ definition given in (3.47) are compared to Monte Carlo simulations generated over 100,000 iterations to evaluate the integral-form expression given in (3.45). Obviously, for large $\lambda$ the analytical results match well with the simulation results and an accuracy of four decimal points is achieved for $\lambda>15$ with all the power constraint scenarios. For small $\lambda$ the few number of terms required to evaluate $\sum_{n=0}^{\infty}(-1)^{n} b_{n}$ makes the infinite-series expressions more appropriate to compute $\bar{P}_{f}$ and $\bar{P}_{d}$ due to their high accuracy. It is also observed that the analytical and the the simulation curves become closer to each other as the power constraint increases which comes from the fact that the errors resulting from Table 3.2: Converging point required to evaluate (3.16) and (3.28) with $\varepsilon$-algorithm. $L=3, m=3$

\begin{tabular}{|c|c|c|c|c|c|}
\cline { 2 - 6 } \multicolumn{1}{c|}{} & \multicolumn{5}{c|}{ Converging Point (n) } \\
\cline { 2 - 6 } \multicolumn{1}{c|}{} & $\lambda=20$ & $\lambda=40$ & $\lambda=60$ & $\lambda=80$ & $\lambda=100$ \\
\hline SC Scheme, Equation(3.16) & 8 & 12 & 16 & 19 & 22 \\
\hline EGC Scheme, Equation(3.28) & 3 & 7 & 10 & 13 & 16 \\
\hline
\end{tabular}


approximating $N_{0}\left(1+\left(1+\bar{\gamma}_{s_{i}}\right) A_{i}\right)$ into $N_{0}\left(1+\bar{\gamma}_{s_{i}}\right) A_{i}$ decrease as $P$ increases.

In Fig. 3.3 we compare the analytical results obtained for $\bar{P}_{d}$ using the exact expressions in (3.16) and (3.28) and the approximated expressions in (3.39) and (3.42) to Monte Carlo simulation results obtained over 100,000 iterations. Generally, the analytical results match well with the simulated ones for both schemes. However,as $\lambda$ increases, the infinite sum requires the evaluation of $\sum_{n=0}^{\infty}(-1)^{n} b_{n}$ over large $n$. Having an insufficient number of terms to evaluate $\sum_{n=0}^{\infty}(-1)^{n} b_{n}$ results in larger convergence errors which explains the slight divergence of the exact curve of the SC scheme from the simulation curve as $\lambda$ increases. Compared with the EGC scheme, the approximated curve of the SC scheme shows a wider mismatch with the simulated one specifically, at small $\lambda$ This mismatch is expected as the approximation errors in the case of EGC scheme are less than that of the SC scheme.

In Fig. 3.4, we describe the receiver's performance through its complementary receiver operating characteristic (ROC) curves. The performance of the proposed AF approach is evaluated using the expression given in (3.28) and compared with a DF approach for different channel conditions. For the DF approach, a false alarm probability and a detection probability are computed at each CR user by $P_{f_{i}}=\Gamma\left(m_{i}, \frac{m_{i} \lambda_{i}}{N_{0}}\right) / \Gamma\left(m_{i}\right)$ and $P_{d_{i}}=\Gamma\left(m_{i}, \frac{m_{i} \lambda_{i}}{N_{0}\left(1+\bar{\gamma}_{s_{i}}\right)}\right) / \Gamma\left(m_{i}\right)$, respectively. Where, $\lambda_{i}$ is the local decision threshold. The cooperative decision is then computed at the fusion center using a logic-based OR-rule. A similar approach is introduced in [29]. The figure illustrates that the AF approach outperforms the DF approach for the selected values of the fading parameter $m$. The 


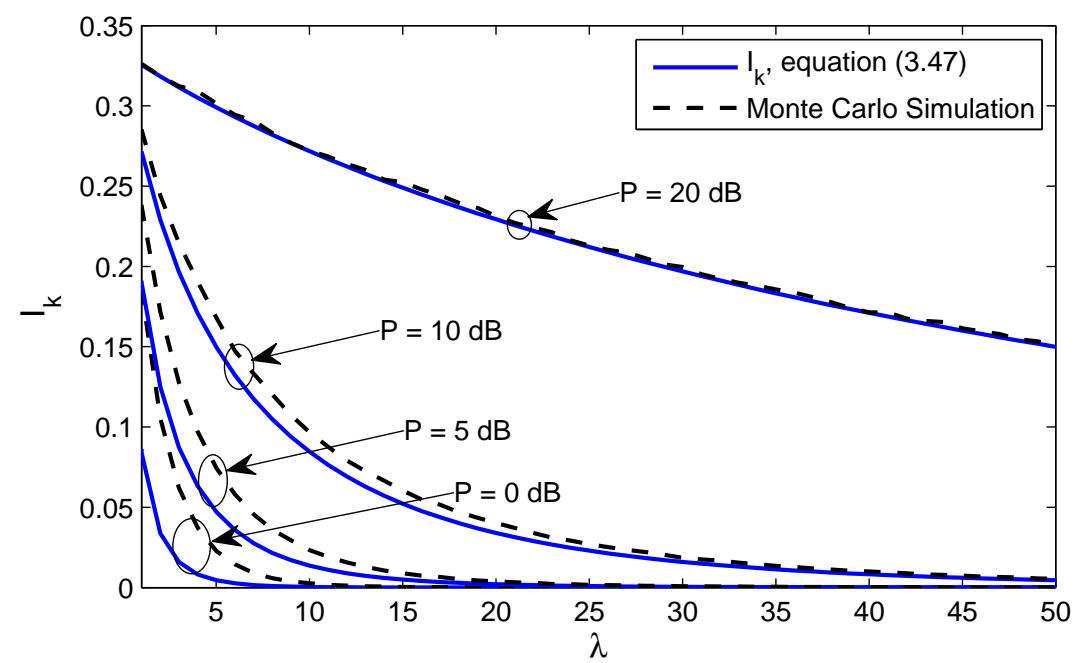

Figure 3.2: Comparison of analytical results of expression (3.47) with Monte Carlo simulations. $L=3, m=3$.

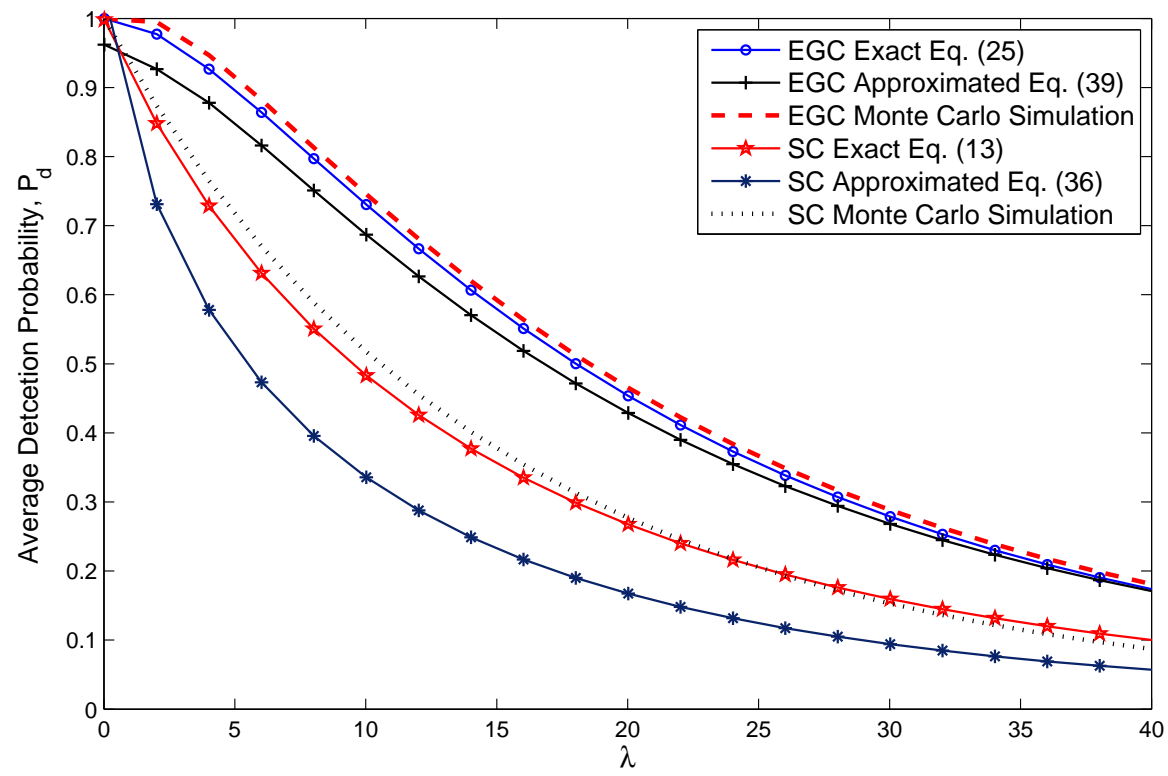

Figure 3.3: Comparison of analytical and simulated $\bar{P}_{d} . P=5 \mathrm{~dB}, L=3, m=2, \bar{\gamma}_{s}=0$ $\mathrm{dB}$. 
AWGN case is also plotted for comparison purposes. AWGN channels are frequently assumed in literature to investigate the energy detection performance [24, 46, 48, 111]. Clearly, such an assumption overestimates the detection accuracy as can be seen from the plotted curves.

In Fig. 3.5, we plot the detection probability of the SC scheme (dot-line curves) and the EGC scheme (solid-line curves) for deferent values of the sensing channel average SNR, $\bar{\gamma}_{s}$. The average detection probability of the SC and EGC schemes are computed using the exact expressions given in (3.16) and (3.28), respectively. There is an obvious improvement in the performance of both combining schemes with each step of $5 \mathrm{~dB}$ increase in $\bar{\gamma}_{s}$ from $-10 \mathrm{~dB}$ to $10 \mathrm{~dB}$. The figure shows that the EGC scheme outperforms the SC scheme for all the selected values of $\bar{\gamma}_{s}$. For low SNR applications, it is more appropriate to the EGC schemes due to the significant difference in its performance compared to the SC scheme as can be seen from the plotted curves.

Fig. 3.6 shows that the detection accuracy is significantly improved by increasing the power constraint, $P$. The average detection probability is plotted for three Nakagami- $m$ fading scenarios with the case $m=1$ corresponding to Rayleigh fading. For $m=6$, a value of $\bar{P}_{d}>0.9$ is achieved when $P$ increases to $8 \mathrm{~dB}$. This matches the target value of $\bar{P}_{d}=0.9$ which is frequently used in literature as a lower bound to the detection probability [107]. This target value is also achieved for other $m$ scenarios but with higher power constraints. The high performance achieved with higher values of the fading parameter, $m$, refers to the fading severity inversely proportional with $m$. Furthermore, 


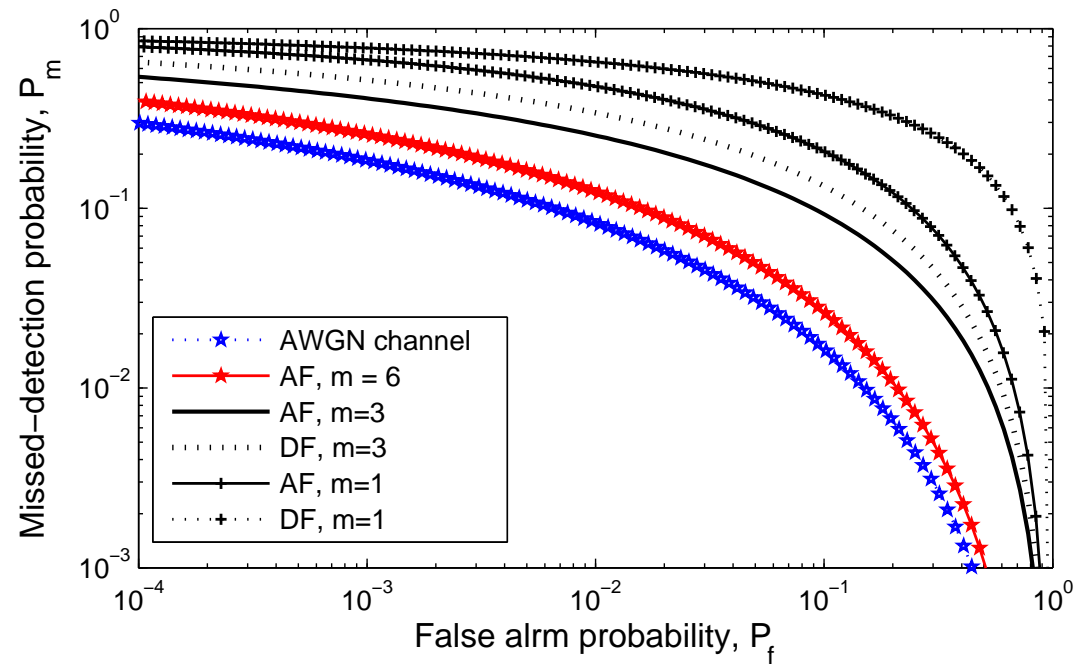

Figure 3.4: Complementary ROC curves at different relaying channel conditions. $P=5$ $\mathrm{dB}, L=3, \bar{\gamma}_{s}=0 \mathrm{~dB}$.

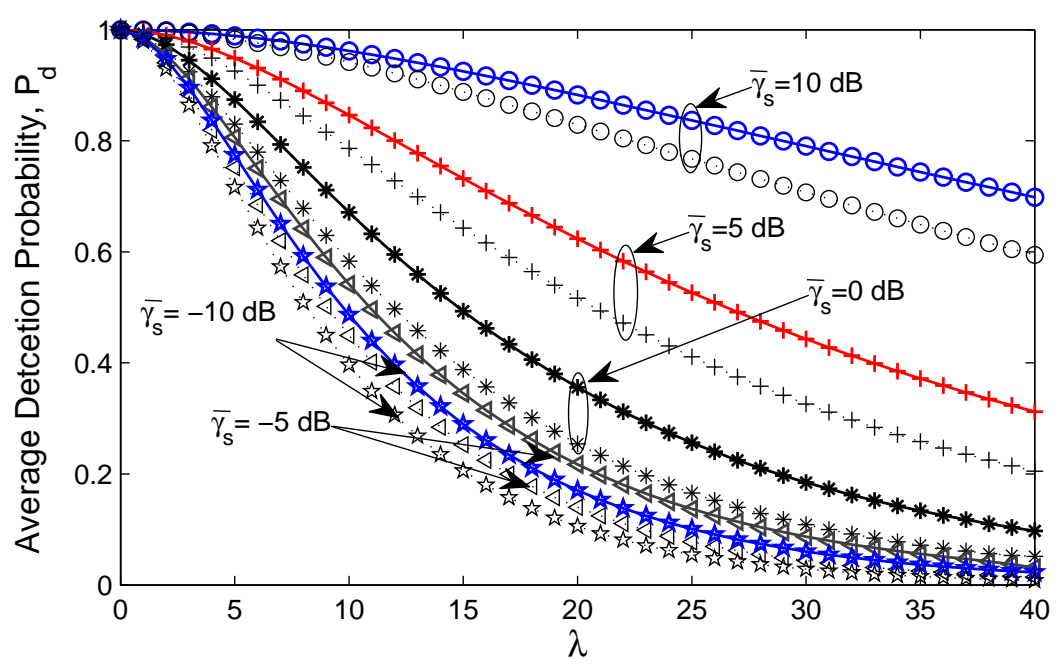

Figure 3.5: Average detection probability for different level of sensing channel SNR. $P=5 \mathrm{~dB}, L=3, m=2$. 
the EGC scheme shows a better performance compared to the SC scheme, specifically at the low power region. Roughly, a gain of $3 \mathrm{~dB}$ is achieved within the range 3 to $10 \mathrm{~dB}$ of the power constraint for $m=3$ when the combiner is switched from the SC scheme to the EGC scheme, yet the SC performance becomes very close to the EGC performance at higher values of $m$. Beyond the target value, the curves slow down as $\bar{P}_{d}$ approaches unity. Thus, there is no need to increase the transmission power to push $\bar{P}_{d}$ beyond this target value, since the higher transmission power leads to a higher interference level that may affect the operation of the primary network.

To demonstrate the importance of the diversity reception, we plot $\bar{P}_{d}$ versus $P$ in Fig. 3.7 for a different number of cooperative users. The expressions of $\bar{P}_{d}$ given in (3.16) and (3.28) are used with the number of diversity branches varies from 1 to 4 . The case of $L=1$ corresponds to the no-diversity scenario. Observe, that for the no-diversity case, the curve of the EGC scheme coincides with that of the SC schemes. The plotted curves indicate that a higher diversity gain is achieved when the number of cooperative users increases. For instance, with $L=4$ users, the EGC receiver achieves $\bar{P}_{d}>0.9$ with $8 \mathrm{~dB}$ less than the no-diversity case while the SC scheme achieves $3 \mathrm{~dB}$ gain with the same number of cooperative users. For the dual branch case, a gain of $4 \mathrm{~dB}$ is achieved with the EGC scheme and $2 \mathrm{~dB}$ with the SC scheme. Furthermore, the EGC scheme outperforms the SC scheme for all the investigated scenarios. 


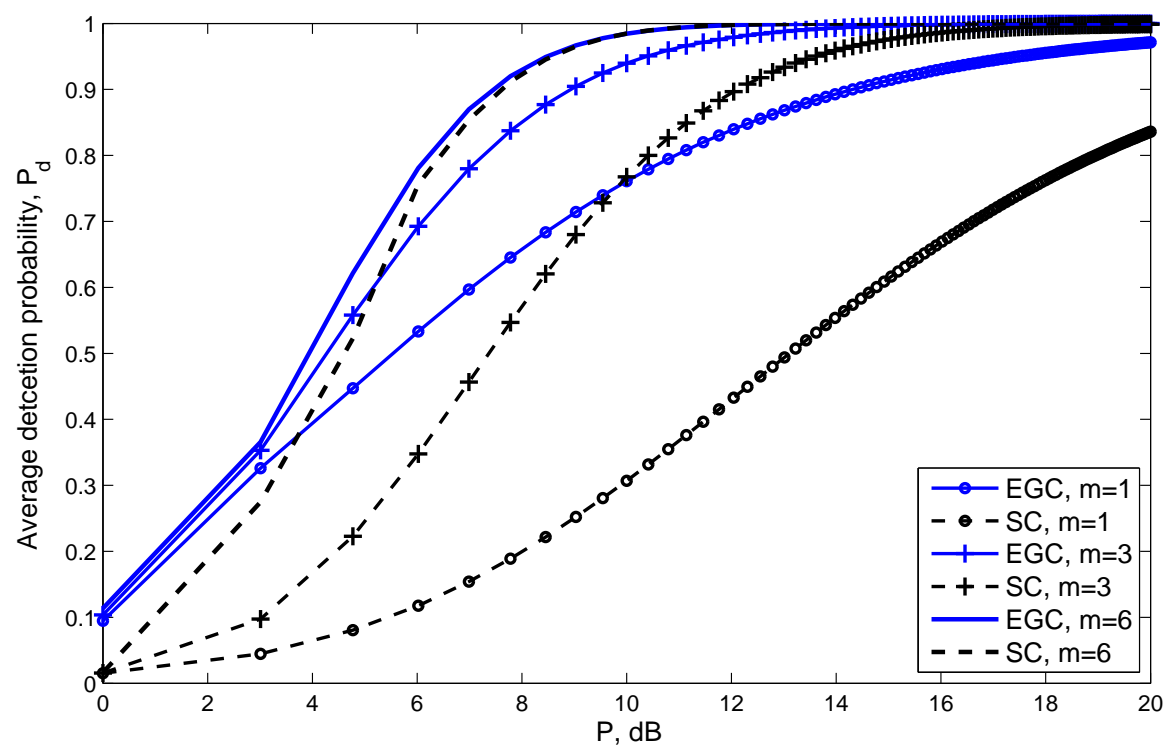

Figure 3.6: Average detection probability vs. relay maximum transmitting power, $\mathrm{P}$. $L=3, \lambda=20, \bar{\gamma}_{s}=0 \mathrm{~dB}$

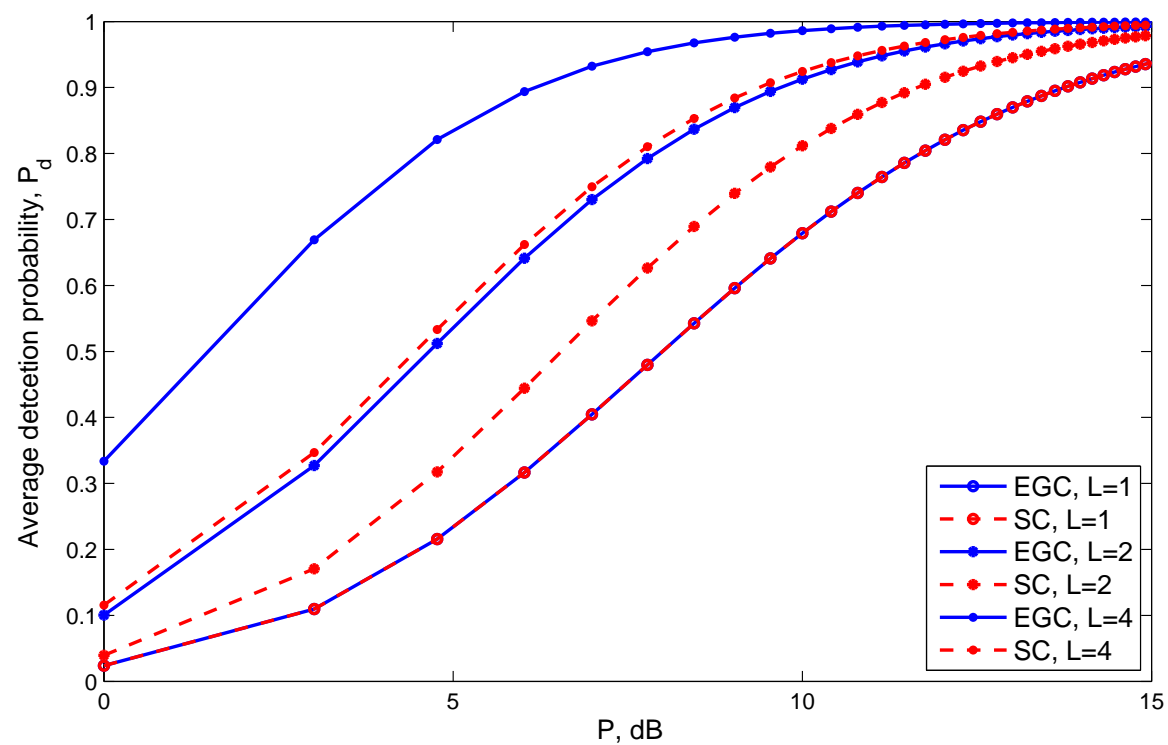

Figure 3.7: Average detection probability vs. relay power constraint, P. $m=3, \lambda=20$, $\bar{\gamma}_{s}=0 \mathrm{~dB}$ 


\section{Chapter 4}

\section{Detect-Amplify-and-Forward}

\section{Strategy over Non-Identical Fading}

\section{Channels}

\subsection{Introduction}

This chapter presents a DAF strategy for relay-based CR networks and investigates its performance over independent non-identically distributed Nakagami- $m$ fading. We aim to mitigate the problem of bandwidth requirements which arises due to reporting real measurements to the fusion center. New exact and approximated closed-form expressions for average detection probability and average false alarm probability are derived for the proposed DAF-based CSS models. The study quantifies the performance of the energy 
detector using detection probability as the main performance measure.

\subsection{Spectrum Sensing Model}

A centralized CR network with $L$ active secondary users is considered. The cooperative decision is assumed to be made by a fusion center. The spectrum sensing phase consists of two time slots. In the first slot, all the $C R_{i}$ s listen to the primary user signal over the shared spectrum band, and in the second slot, each CR performs a local detection based on the signal received in the first slot. If the decision is that no primary's signal is detected, the CR keeps quiet during the second slot [111]. Otherwise, If the primary's signal is detected, the $\mathrm{CR}$ amplifies the received signal and relays to the fusion center by the maximum transmission power constraint. We assume that CSI is available for all the relays. Also, the fusion center has a full knowledge of CSI, i.e., it can acquire the global knowledge of sensing and relaying channel gains.

\subsubsection{Single-Relay System}

According to the DAF strategy, each CR user performs a local detection and makes its own decision $\hat{\theta}_{i}$ regarding the primary user status. Then, if $\hat{\theta}_{i}=1$, the CR user amplifies and relays its local sensing to the fusion center. Otherwise, it keeps quiet if $\hat{\theta}_{i}=0$. Accordingly, the signal received at the fusion center over the $i$ th relaying link takes the 
form $[21]$

$$
y_{i}=\hat{\theta}_{i} h_{r_{i}}\left(\sqrt{A_{i}}\left(\theta h_{s_{i}} x_{p}+n_{s_{i}}\right)\right)+n_{r_{i}}=\theta \hat{\theta}_{i} \sqrt{A_{i}} h_{r_{i}} h_{s_{i}} x_{p}+\hat{\theta}_{i} \sqrt{A_{i}} h_{r_{i}} n_{s_{i}}+n_{r_{i}}
$$

and

$$
\mathrm{E}\left\{Y_{i} \mid g\right\}= \begin{cases}\sigma_{Y_{i_{0}}}=N_{0}\left(1+\hat{\theta}_{i} A_{i} g\right) & H_{0} \\ \sigma_{Y_{i_{1}}}=N_{0}\left(1+\hat{\theta}_{i}\left(1+\bar{\gamma}_{s_{i}}\right) A_{i} g\right) & H_{1}\end{cases}
$$

Now, the false alarm probability for a given $g$ can be expressed as

$$
\begin{aligned}
P_{f_{i} \mid g}^{D A F}= & P\left\{Y_{i} \geq \lambda \mid H_{0}, g\right\}=P\left\{Y_{i} \geq \lambda \mid \hat{\theta}_{i}=1, H_{0}\right\} \cdot P\left\{\hat{\theta}_{i}=1 \mid H_{0}\right\} \\
& +P\left\{Y_{i} \geq \lambda \mid \hat{\theta}_{i}=0, H_{0}\right\} \cdot P\left\{\hat{\theta}_{i}=0 \mid H_{0}\right\} \\
= & P_{f_{i}} \cdot P\left\{Y_{i} \geq \lambda \mid \hat{\theta}_{i}=1, H_{0}\right\}+\left(1-P_{f_{i}}\right) \cdot P\left\{Y_{i} \geq \lambda \mid \hat{\theta}_{i}=0, H_{0}\right\} .
\end{aligned}
$$

Similarly, the detection probability can be expressed as

$$
\begin{aligned}
P_{d_{i} \mid g}^{D A F}= & P\left\{Y_{i} \geq \lambda \mid H_{1}, g\right\}=P\left\{Y_{i} \geq \lambda \mid \hat{\theta}_{i}=1, H_{1}\right\} \cdot P\left\{\hat{\theta}_{i}=1 \mid H_{1}\right\} \\
& +P\left\{Y_{i} \geq \lambda \mid \hat{\theta}_{i}=0, H_{1}\right\} \cdot P\left\{\hat{\theta}_{i}=0 \mid H_{1}\right\} \\
= & P_{d_{i}} \cdot P\left\{Y_{i} \geq \lambda \mid \hat{\theta}_{i}=1, H_{1}\right\}+\left(1-P_{d_{i}}\right) \cdot P\left\{Y_{i} \geq \lambda \mid \hat{\theta}_{i}=0, H_{1}\right\} .
\end{aligned}
$$

From (4.1) and (4.3), the PDF of $Y_{i}$ under hypothesis $H_{0}$ denoted by $f_{Y_{i} \mid H_{0}}(y)$ for a 
given $g$ is given by

$$
\begin{aligned}
f_{Y_{i} \mid H_{0}}(y) & =f_{Y_{i} \mid H_{0}, \hat{\theta}_{i}=0}(y) \cdot P\left\{\hat{\theta}_{i}=0 \mid H_{0}\right\}+f_{Y_{i} \mid H_{0}, \hat{\theta}_{i}=1}(y) \cdot P\left\{\hat{\theta}_{i}=1 \mid H_{0}\right\} \\
& =\sum_{\hat{\theta}_{i}=0}^{\hat{\theta}_{i}=1} P_{f_{i}}^{\hat{\theta}_{i}}\left(1-P_{f_{i}}\right)^{1-\hat{\theta}_{i}}\left(\frac{m_{i}}{\sigma_{Y_{i_{0}}}}\right)^{m_{i}} \frac{1}{\Gamma\left(m_{i}\right)} y^{m_{i}-1} e^{\left(-\frac{m_{i}}{\sigma_{Y_{i}}}\right) y}, \quad y \geq 0 .
\end{aligned}
$$

Similarly, From (4.1) and (4.4), the PDF of $Y_{i}$ under hypothesis $H_{1}$ denoted by $f_{Y_{i} \mid H_{1}}(y)$ for a given $g$ is given by

$$
\begin{aligned}
f_{Y_{i} \mid H_{1}}(y) & =f_{Y_{i} \mid H_{1}, \hat{\theta}_{i}=0}(y) \cdot P\left\{\hat{\theta}_{i}=0 \mid H_{1}\right\}+f_{Y_{i} \mid H_{1}, \hat{\theta}_{i}=1}(y) \cdot P\left\{\hat{\theta}_{i}=1 \mid H_{1}\right\} \\
& =\sum_{\hat{\theta}_{i}=0}^{\hat{\theta}_{i}=1} P_{d_{i}}^{\hat{\theta}_{i}}\left(1-P_{d_{i}}\right)^{1-\hat{\theta}_{i}}\left(\frac{m_{i}}{\sigma_{Y_{i_{1}}}}\right)^{m_{i}} \frac{1}{\Gamma\left(m_{i}\right)} y^{m_{i}-1} e^{\left(-\frac{m_{i}}{\sigma_{Y_{i_{1}}}}\right) y}, \quad y \geq 0 .
\end{aligned}
$$

Then the average false alarm probability $\bar{P}_{f_{i}}^{D A F}$ can be evaluated using the channel statistic given in (3.7) as follows

$$
\begin{aligned}
\bar{P}_{f_{i}}^{D A F} & =\int_{0}^{\infty} P\left\{Y_{i}>\lambda \mid H_{0}, g\right\} f_{G_{i}}(g) d g \\
& =\sum_{\hat{\theta}_{i}=0}^{\hat{\theta}_{i}=1} P_{f_{i}}^{\hat{\theta}_{i}}\left(1-P_{f_{i}}\right)^{1-\hat{\theta}_{i}} \int_{0}^{\infty} \frac{1}{\Gamma\left(m_{i}\right)} \Gamma\left(m_{i}, \frac{m_{i} \lambda}{\sigma_{Y_{i}}}\right) f_{G_{i}}(g) d g \\
& =\sum_{\hat{\theta}_{i}=0}^{\hat{\theta}_{i}=1} P_{f_{i}}^{\hat{\theta}_{i}}\left(1-P_{f_{i}}\right)^{1-\hat{\theta}_{i}} \int_{0}^{\infty} \frac{1}{\Gamma\left(m_{i}\right)} \Gamma\left(m_{i}, \frac{m_{i} \lambda}{N_{0}\left(1+A_{i} g\right)}\right) f_{G_{i}}(g) d g .
\end{aligned}
$$

Note that, a false alarm means a relaying state under $H_{0}$ hypothesis. In the case, $\hat{\theta}_{i}=1$ and $\sigma_{Y_{i_{0}} \mid H_{0}, \hat{\theta}_{i}=1}=N_{0}\left(1+A_{i} g\right)$ as can be seen in the expression given in (4.7). For computational simplicity, let $\mathbb{P}_{i \mid H_{0}}=P_{f_{i}}^{\hat{\theta}_{i}}\left(1-P_{f_{i}}\right)^{1-\hat{\theta}_{i}}$, then in a similar way to derive 
$\bar{P}_{f_{i}}^{A F}, \bar{P}_{f_{i}}^{D A F}$ can be mathematically expressed as

$$
\bar{P}_{f_{i}}^{D A F}=\sum_{\hat{\theta}_{i}=0}^{\hat{\theta}_{i}=1} \mathbb{P}_{i \mid H_{0}}\left(\frac{\beta_{i}}{A_{i}}\right)^{m_{i}} \sum_{q=0}^{m_{i}-1}\left(\frac{m_{i} \lambda_{i}}{N_{0}}\right)^{q} \frac{1}{q !} \sum_{n=0}^{\infty}(-1)^{n} b_{n} U\left(m_{i} ; m_{i}+1-q-n ; \frac{\beta_{i}}{A_{i}}\right) .
$$

Similarly, the average detection probability $\bar{P}_{d_{i}}^{D A F}$ can be expressed as

$\bar{P}_{d_{i}}^{D A F}=\sum_{\hat{\theta}_{i}=0}^{\hat{\theta}_{i}=1} \mathbb{P}_{i \mid H_{1}}\left(\frac{\beta_{i}}{\left(1+\bar{\gamma}_{s_{i}}\right) A_{i}}\right)^{m_{i}} \sum_{q=0}^{m_{i}-1}\left(\frac{m_{i} \lambda_{i}}{N_{0}}\right)^{q} \frac{1}{q !} \sum_{n=0}^{\infty}(-1)^{n} b_{n} U\left(m_{i} ; m_{i}+1-q-n ; \frac{\beta_{i}}{\left(1+\bar{\gamma}_{s_{i}}\right) A_{i}}\right)$

where, $\mathbb{P}_{i \mid H_{1}}=P_{d_{i}}^{\hat{\theta}_{i}}\left(1-P_{d_{i}}\right)^{1-\hat{\theta}_{i}}$

\subsubsection{Multi-Relay System}

In this section, we consider the DAF strategy in a multi-relay system. Each CR independently makes its own decision then amplifies and forwards to the fusion center only when it claims the activity of the primary user. The fused signal can be expressed as

$$
y=\sum_{i=1}^{L} \hat{\theta}_{i} \sqrt{A_{i}} h_{r_{i}}\left(\theta h_{s_{i}} x_{p}+n_{s_{i}}\right)+n_{r_{i}}=\theta_{i} \sum_{i=1}^{L} \hat{\theta}_{i} \sqrt{A_{i}} h_{r_{i}} h_{s_{i}} x_{P}+\sum_{i=1}^{L} \hat{\theta}_{i} \sqrt{A_{i}} h_{r_{i}} n_{s_{i}}+n_{r_{i}} .
$$

From (4.10), for given $g_{i}$ 's, the mean value of $Y$ can be expressed as

$$
\mathrm{E}\left\{Y \mid g_{i}{ }^{\prime} s\right\}= \begin{cases}\sigma_{Y_{0}}=N_{0}\left(1+\sum_{i=1}^{L} \hat{\theta}_{i} A_{i} g_{i}\right) & H_{0} \\ \sigma_{Y_{1}}=N_{0}\left(1+\sum_{i=1}^{L}\left(1+\bar{\gamma}_{s_{i}}\right) \hat{\theta}_{i} A_{i} g_{i}\right) & H_{1} .\end{cases}
$$

Using (4.11), the PDF of $Y$ under the hypothesis $H_{0}$ denoted by $f_{Y \mid H_{0}}(y)$ for given 
$g_{i}$ 's can be written as

$$
\begin{aligned}
f_{Y \mid H_{0}}(y)= & f_{Y \mid H_{0}, \hat{\theta}_{1}=0, \cdots, \hat{\theta}_{L}=0}(y) \cdot P\left\{\hat{\theta}_{1}=0, \cdots, \hat{\theta}_{L}=0 \mid H_{0}\right\}+\cdots \\
& +f_{Y \mid H_{0}, \hat{\theta}_{1}=1, \cdots, \hat{\theta}_{L}=1}(y) \cdot P\left\{\hat{\theta}_{1}=1, \cdots, \hat{\theta}_{L}=1 \mid H_{0}\right\} \\
= & \sum_{\hat{\theta}_{1}=0}^{\hat{\theta}_{1}=1} \cdots \sum_{\hat{\theta}_{L}=1}^{L} \prod_{i=1}^{L} P_{f_{i}}^{\hat{\theta}_{i}}\left(1-P_{f_{i}}\right)^{\hat{\theta}_{i}}\left(\frac{m_{i}}{\sigma_{Y_{0}}}\right)^{m_{i}} \frac{1}{\Gamma\left(m_{i}\right)} y^{m_{i}-1} e^{\left(-\frac{m_{i}}{\sigma_{Y_{0}}}\right) y}, \quad y \geq 0
\end{aligned}
$$

and the PDF of $Y$ under $H_{1}$ denoted by $f_{Y \mid H_{1}}(y)$ can be written as

$$
\begin{aligned}
f_{Y \mid H_{1}}(y)= & f_{Y \mid H_{1}, \hat{\theta}_{1}=0, \cdots, \hat{\theta}_{L}=0}(y) \cdot P\left\{\hat{\theta}_{1}=0, \cdots, \hat{\theta}_{L}=0 \mid H_{1}\right\}+\cdots \\
& +f_{Y \mid H_{1}, \hat{\theta}_{1}=1, \cdots, \hat{\theta}_{L}=1}(y) \cdot P\left\{\hat{\theta}_{1}=1, \cdots, \hat{\theta}_{L}=1 \mid H_{1}\right\} \\
= & \sum_{\hat{\theta}_{1}=0} \cdots \sum_{\hat{\theta}_{L}=0}^{\hat{\theta}_{L}=1} \prod_{i=1}^{L} P_{d_{i}}^{\hat{\theta}_{i}}\left(1-P_{d_{i}}\right)^{\hat{\theta}_{i}}\left(\frac{m_{i}}{\sigma_{Y_{1}}}\right)^{m_{i}} \frac{1}{\Gamma\left(m_{i}\right)} y^{m_{i}-1} e^{\left(-\frac{m_{i}}{\sigma_{Y_{1}}}\right) y}, \quad y \geq 0 .
\end{aligned}
$$

Obviously, each of $f_{Y \mid H_{0}}(y)$ and $f_{Y \mid H_{1}}(y)$ consists of $2^{L}$ cases that relate to the decisions $\hat{\theta}_{i}$ 's as can be seen from $(4.12)$ and $(4.13)$, respectively. We define $\hat{\theta}^{j}=\left[\hat{\theta}_{1}^{j}, \cdots, \hat{\theta}_{L}^{j}\right]$ to denote the decision vector of the $j$ th case, $j \in\left[1,2^{L}\right]$. It is clear that $f_{Y \mid H_{0}}(y)$ and $f_{Y \mid H_{1}}(y)$ can be written as a combination of Nakagami- $m$ distributions, which is composed of $2^{L}$ sub-populations in proportions $\prod_{i=1}^{L} \mathbb{P}_{i}^{j}\left(\prod_{i=1}^{L} \mathbb{P}_{i}^{1}+\prod_{i=1}^{L} \mathbb{P}_{i}^{2}+\prod_{i=1}^{L} \mathbb{P}_{i}^{3}+\cdots+\prod_{i=1}^{L} \mathbb{P}_{i}^{2^{L}}=1\right)$ 
as follows

$$
\begin{aligned}
& f_{Y \mid H_{0}}(y)=\sum_{j=1}^{2^{L}} \prod_{i=1}^{L} \mathbb{P}_{i \mid H_{0}}^{j}\left(\frac{m_{i}}{\sigma_{Y_{0}}}\right)^{m_{i}} \frac{1}{\Gamma\left(m_{i}\right)} y^{m_{i}-1} e^{\left(-\frac{m_{i}}{\sigma_{Y_{0}}}\right) y}, \quad y \geq 0 \\
& f_{Y \mid H_{1}}(y)=\sum_{j=1}^{2^{L}} \prod_{i=1}^{L} \mathbb{P}_{i \mid H_{1}}^{j}\left(\frac{m_{i}}{\sigma_{Y_{1}}}\right)^{m_{i}} \frac{1}{\Gamma\left(m_{i}\right)} y^{m_{i}-1} e^{\left(-\frac{m_{i}}{\sigma_{Y_{1}}}\right) y}, \quad y \geq 0
\end{aligned}
$$

where, $\mathbb{P}_{i \mid H_{0}}^{j}=P_{f_{i}}^{\hat{\theta}_{i}^{j}}\left(1-P_{f_{i}}\right)^{1-\hat{\theta}_{i}^{j}}$ and $\mathbb{P}_{i \mid H_{1}}^{j}=\prod_{i=1}^{L} P_{d_{i}}^{\hat{\theta}_{i}^{j}}\left(1-P_{d_{i}}\right)^{1-\hat{\theta}_{i}^{j}}$ denote the occurrence probability of the $j$ th case under $H_{0}$ and $H_{1}$, respectively. Therefore, the average detection probability $\bar{P}_{f}^{D A F}$, can be evaluated by averaging over all the possible relaying links using $f_{G}(g)$ given in $(3.7)$

$$
\begin{aligned}
\bar{P}_{f}^{D A F}= & P\left\{Y \geq \lambda \mid H_{0}\right\}=\underbrace{\int_{0}^{\infty} \cdots \int_{0}^{\infty}}_{i=1, \cdots, L, \hat{\theta}_{i}^{j} \neq 0} P\left\{Y \geq \lambda \mid H_{0}, g_{1}, \cdots g_{L}\right\} \cdot f\left(g_{1} \mid H_{0}\right) \cdots f\left(g_{L} \mid H_{0}\right) d g_{1} \cdots d g_{L} \\
= & \sum_{j=1}^{2^{L}}\left[\prod_{i=1}^{L} \mathbb{P}_{i \mid H_{0}}^{j}\right] \underbrace{\int_{0}^{\infty} \cdots \int_{0}^{\infty}}_{i=1, \cdots, L, \hat{\theta}_{i}^{j} \neq 0} \frac{1}{\Gamma\left(m_{i}\right)} \Gamma\left(m_{i}, \frac{m_{i} \lambda}{N_{0}\left(1+\sum_{k=1, \hat{\theta}_{k}^{j} \neq 0}^{L} A_{k} g_{k}\right)}\right) f\left(g_{1} \mid H_{0}\right) \cdots f\left(g_{L} \mid H_{0}\right) \\
& d g_{1} \cdots d g_{L} .
\end{aligned}
$$

In a similar way to $\bar{P}_{f}^{A F}$ derivation, we define the following random variable,

$$
R=\sum_{k=1, \hat{\theta}_{k}^{j} \neq 0}^{L} A_{k} g_{k} .
$$


Therefore, the expression of $\bar{P}_{f}^{D A F}$ in (4.16) can be expressed as,

$$
\bar{P}_{f}^{D A F}=\sum_{j=1}^{2^{L}}\left[\prod_{i=1}^{L} \mathbb{P}_{i \mid H_{0}}^{j}\right] \int_{0}^{\infty} \frac{1}{\Gamma\left(m_{i}\right)} \Gamma\left(m_{i}, \frac{m_{i} \lambda}{N_{0}(1+r)}\right) f_{R}(r) d r
$$

Using the same steps as in Appendix A.3, $f_{R}(r)$, can be expressed as

$$
f_{R}(r)=\left[\prod_{l=1, \hat{\theta}_{l}^{j} \neq 0}^{L}\left(-\frac{\beta_{l}}{A_{l}}\right)^{m_{l}}\right] \sum_{i=1, \hat{\theta}_{i}^{j} \neq 0}^{L} \sum_{v=1}^{m_{i}} \frac{(-1)^{v} b_{i v} r^{v-1} e^{-\frac{m_{i}}{\bar{g}_{i} A_{i}} r}}{(v-1) !} .
$$

Substituting (4.19) into (4.18) yields

$$
\begin{aligned}
\bar{P}_{f}^{D A F}= & \sum_{j=1}^{2^{L}}\left[\prod_{i=1}^{L} \mathbb{P}_{i \mid H_{0}}^{j}\right]\left[\prod_{l=1, \hat{\theta}_{l}^{j} \neq 0}^{L}\left(-\frac{\beta_{l}}{A_{l}}\right)^{m_{l}}\right] \sum_{i=1, \hat{\theta}_{i}^{j} \neq 0}^{L} \sum_{v=1}^{m_{i}} \frac{(-1)^{v} b_{i \nu}}{\left(m_{i}-v\right) !} \frac{1}{\Gamma\left(m_{i}\right)} \int_{0}^{\infty} \Gamma\left(m_{i}, \frac{m_{i} \lambda}{N_{0}(1+r)}\right) \\
& r^{v-1} e^{-\frac{\beta_{i}}{A_{i} r} d r .}
\end{aligned}
$$

Following the same steps in Appendix B.1 to evaluate the above integral, (4.20) becomes,

$$
\begin{aligned}
\bar{P}_{f}^{D A F}= & \sum_{j=1}^{2^{L}}\left[\prod_{i=1}^{L} \mathbb{P}_{i \mid H_{0}}^{j}\right]\left[\prod_{l=1, \hat{\theta}_{l}^{j} \neq 0}^{L}\left(-\frac{\beta_{l}}{A_{l}}\right)^{m_{l}}\right] \sum_{i=1, \hat{\theta}_{i}^{j} \neq 0}^{L} \sum_{v=1}^{m_{i}}(-1)^{v} b_{i v} \sum_{q=0}^{m_{i}-1} \frac{1}{q !}\left(\frac{m_{i} \lambda}{N_{0}}\right)^{q} \\
& \sum_{n=0}^{\infty}(-1)^{n} b_{n} U\left(v ; v+1-q-n ; \frac{\beta_{i}}{A_{i}}\right)
\end{aligned}
$$


Similarly, the average detection probability can be expressed as

$$
\begin{aligned}
\bar{P}_{d}^{D A F}= & \sum_{j=1}^{2^{L}}\left[\prod_{i=1}^{L} \mathbb{P}_{i \mid H_{1}}^{j}\right]\left[\prod_{l=1, \hat{\theta}_{l}^{j} \neq 0}^{L}\left(-\frac{\beta_{l}}{\left(1+\bar{\gamma}_{s_{l}}\right) A_{l}}\right)^{m_{l}}\right] \sum_{i=1, \hat{\theta}_{i}^{j} \neq 0}^{L} \sum_{v=1}^{m_{i}}(-1)^{v} b_{i v} \sum_{q=0}^{m_{i}-1} \frac{1}{q !}\left(\frac{m_{i} \lambda}{N_{0}}\right)^{q} \\
& \sum_{n=0}^{\infty}(-1)^{n} b_{n} U\left(v ; v+1-q-n ; \frac{\beta_{i}}{\left(1+\bar{\gamma}_{s_{i}}\right) A_{i}}\right) .
\end{aligned}
$$

For Rayleigh fading channels, $\bar{P}_{f_{\text {Ray }}}^{D A F}$ and $\bar{P}_{d_{\text {Ray }}}^{D A F}$ are evaluated by setting $m_{i}=1, i=$ $1, \cdots, L$ in $(4.21)$ and $(4.22)$

$$
\bar{P}_{f_{\text {Ray }}}^{D A F}=(-1)^{\sum_{k=1, \hat{\theta}_{i}^{j} \neq 0}^{L} k+1} \sum_{j=1}^{2^{L}}\left[\prod_{i=1}^{L} \mathbb{P}_{i \mid H_{0}}^{j}\right]\left[\prod_{l=1, \hat{\theta}_{l}^{j} \neq 0}^{L} \frac{1}{A_{l} \bar{g}_{l}}\right] \sum_{i=1, \hat{\theta}_{i}^{j} \neq 0}^{L} \tilde{\Delta}_{i} \sum_{n=0}^{\infty}(-1)^{n+1} \tilde{b}_{n} U\left(1 ; 2-n ; \frac{1}{A_{i} \bar{g}_{i}}\right)
$$

and

$$
\begin{aligned}
\bar{P}_{d_{\text {Ray }}}^{D A F}= & (-1)^{\sum_{k=1, \hat{\theta}_{i}^{j} \neq 0}^{k}{ }^{k+1}} \sum_{j=1}^{2^{L}}\left[\prod_{i=1}^{L} \mathbb{P}_{i \mid H_{1}}^{j}\right]\left[\prod_{l=1, \hat{\theta}_{l}^{j} \neq 0}^{L} \frac{1}{\left(1+\bar{\gamma}_{s_{l}}\right) A_{l} \bar{g}_{l}}\right] \sum_{i=1, \hat{\theta}_{i}^{i} \neq 0}^{L} \tilde{\Delta}_{i} \sum_{n=0}^{\infty}(-1)^{n+1} \tilde{b}_{n} \\
& U\left(1 ; 2-n ; \frac{1}{\left(1+\bar{\gamma}_{s_{i}}\right) A_{i} \bar{g}_{i}}\right) .
\end{aligned}
$$

\subsubsection{Approximated Analysis}

Similar to AF strategy, we propose new computational approach to avoid seeing the infinite series in the closed-form expressions derived for the average false alarm probability and the average detection probability. If the value of $A_{i}$ is assumed to be high enough, then we have $A_{i} g \gg 1$ and $\left(1+\bar{\gamma}_{s_{i}}\right) A_{i} g \gg 1$. In the following equations, we present the 
approximated closed-form expressions derived for the average false alarm probability and the average detection probability.

$$
\begin{aligned}
\bar{P}_{f}^{D A F}= & 2 \sum_{j=1}^{2^{L}}\left[\prod_{i=1}^{L} \mathbb{P}_{i \mid H_{0}}^{j}\right]\left[\prod_{l=1, \hat{\theta}_{l}^{j} \neq 0}^{L}\left(-\frac{\beta_{l}}{A_{l}}\right)^{m_{l}}\right] \sum_{i=1, \hat{\theta}_{i}^{j} \neq 0}^{L} \sum_{v=1}^{m_{i}} \frac{(-1)^{v} b_{i v}}{\Gamma(v)} \sum_{q=0}^{m_{i}-1} \frac{1}{q !}\left(\frac{m_{i} \lambda}{N_{0}}\right)^{\frac{v+q}{2}}\left(\frac{A_{i}}{\beta_{i}}\right)^{\frac{v-q}{2}} \\
& K_{v-q}\left(2 \sqrt{\frac{m_{i} \beta_{i} \lambda}{N_{0} A_{i}}}\right) .
\end{aligned}
$$

$$
\begin{aligned}
\bar{P}_{d}^{D A F}= & 2 \sum_{j=1}^{2^{L}}\left[\prod_{i=1}^{L} \mathbb{P}_{i \mid H_{0}}^{j}\right]\left[\prod_{l=1, \hat{\theta}_{l}^{j} \neq 0}^{L}\left(-\frac{\beta_{l}}{\left(1+\bar{\gamma}_{s_{l}}\right) A_{l}}\right)^{m_{l}}\right] \sum_{i=1, \hat{\theta}_{i}^{j} \neq 0}^{L} \sum_{v=1}^{m_{i}} \frac{(-1)^{v} b_{i v}}{\Gamma(v)} \sum_{q=0}^{m_{i}-1} \frac{1}{q !}\left(\frac{m_{i} \lambda}{N_{0}}\right)^{\frac{v+q}{2}} \\
& \left(\frac{\left(1+\bar{\gamma}_{s_{i}}\right) A_{i}}{\beta_{i}}\right)^{\frac{v-q}{2}} K_{v-q}\left(2 \sqrt{\frac{m_{i} \beta_{i} \lambda}{N_{0}\left(1+\bar{\gamma}_{s_{i}}\right) A_{i}}}\right) .
\end{aligned}
$$

$$
\bar{P}_{f_{\text {Ray }}}^{A F}=2(-1)^{\sum_{k=1, \hat{\theta}_{i}^{j} \neq 0}^{L}{ }^{k+1}} \sum_{j=1}^{2^{L}}\left[\prod_{i=1}^{L} \mathbb{P}_{i \mid H_{0}}^{j}\right]\left[\prod_{l=1, \hat{\theta}_{l}^{j} \neq 0}^{L} \frac{1}{A_{l} \bar{g}_{l}}\right] \sum_{i=1, \hat{\theta}_{i}^{j} \neq 0}^{L} \tilde{\Delta}_{i} \sqrt{\frac{\lambda A_{i} \bar{g}_{i}}{N_{0}}} K_{1}\left(2 \sqrt{\frac{\lambda}{N_{0} A_{i} \bar{g}_{i}}}\right) .
$$

$$
\begin{aligned}
\bar{P}_{d_{\text {Ray }}}^{A F}= & 2(-1)^{\sum_{k=1, \hat{\theta}_{i}^{j} \neq 0}^{L}{ }^{k+1}} \sum_{j=1}^{2^{L}}\left[\prod_{i=1}^{L} \mathbb{P}_{i \mid H_{0}}^{j}\right]\left[\prod_{l=1, \hat{\theta}_{l}^{j} \neq 0}^{L} \frac{1}{\left(1+\bar{\gamma}_{s_{l}}\right) A_{l} \bar{g}_{l}}\right] \sum_{i=1, \hat{\theta}_{i}^{j} \neq 0}^{L} \tilde{\Delta}_{i} \sqrt{\frac{\lambda\left(1+\bar{\gamma}_{s_{i}}\right) A_{i} \bar{g}_{i}}{N_{0}}} \\
& K_{1}\left(2 \sqrt{\frac{\lambda}{N_{0}\left(1+\bar{\gamma}_{s_{i}}\right) A_{i} \bar{g}_{i}}}\right)
\end{aligned}
$$

Proof: See Appendix B.2. 


\subsection{Performance Evaluation}

To validate the accuracy of the derived closed-form expressions and to quantify the performance of the proposed CR network approach, several analytical and numerical simulations are performed with variety of diversity combinations. The noise variance $N_{0}$ as well as $E_{i}$ is set to be unity $(0 \mathrm{~dB})$ and an upper bound of $P_{f_{i}} \leq 0.1$ and a lower bound of $P_{d_{i}} \geq 0.9$ are considered. In Fig. 4.1, analytical results of the exact and the approximated closed-form expressions of $\bar{P}_{d}^{D A F}$ given in (4.22) and (4.26), respectively, are verified by Monte Carlo simulations generated over 100,000 iterations. The Monte Carlo simulations are set to calculate numerically the expression given in (B.1). As can be seen, the analytical results of the exact closed-form expression match well with the simulation results and an accuracy of four decimal points is achieved for $\lambda<15$. Therefore, for small $\lambda$ 's, the exact infinite-series closed-form expressions can be used to compute the average detection probability. The small number of terms required to evaluate the infinite series at small $\lambda$ 's makes those expressions more appropriate in this case. However, when $\lambda$ becomes large, more terms are required to be summed to evaluate the infinite series $\sum_{n=0}^{\infty}(-1)^{n} b_{n}$. Therefore, for large $\lambda$ 's, it is more appropriate to use the approximated expressions for computing $\bar{P}_{d}^{D A F}$ due to their computational simplicity. In Fig. 4.2, the ROC curves of the energy detector are plotted for variety of diversity paths. The curves are plotted for probability of missed detection $\left(1-\bar{P}_{d}\right)$ versus probability of false alarm. Equations (4.21) and (4.22) are used to compute the average false alarm probability and the average detection probability, respectively. A close observation of Fig. 4.2 shows that 


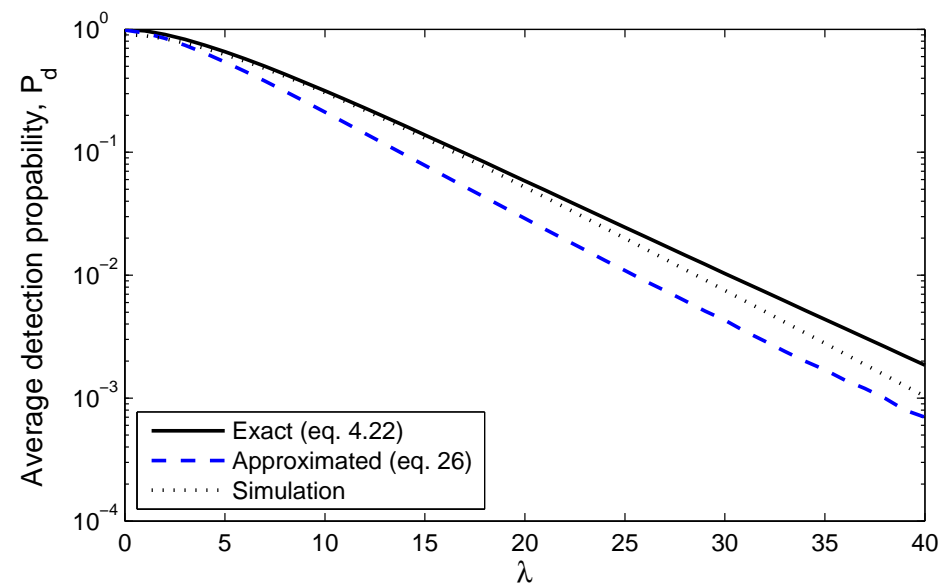

Figure 4.1: Comparison of analytical results of expressions (4.22) and (4.26) with Monte Carlo simulations for $L=3, m=2, P=5 \mathrm{~dB}$, and $\bar{\gamma}_{s}=0 \mathrm{~dB}$.

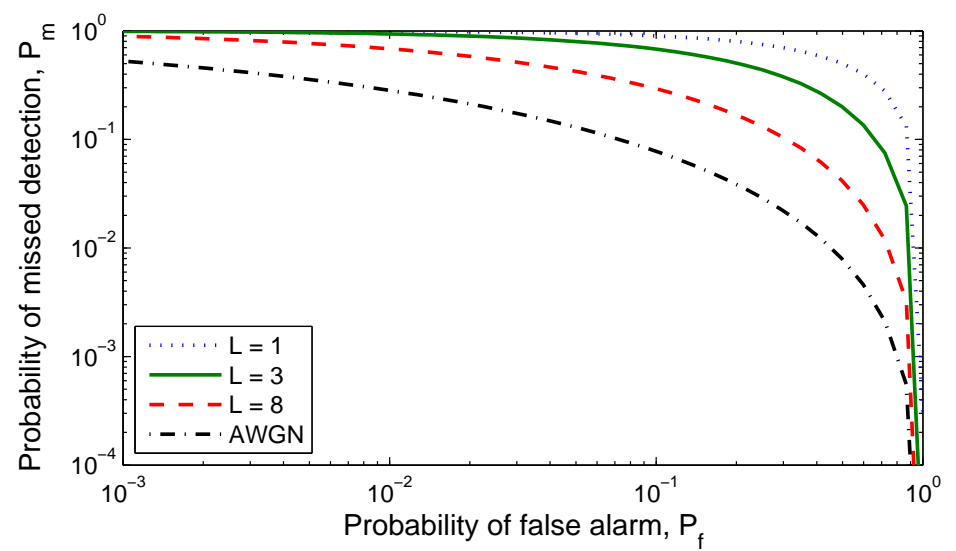

Figure 4.2: ROC curves of the energy detector with $m=2, P=5 \mathrm{~dB}$, and $\bar{\gamma}_{s}=0 \mathrm{~dB}$. 
the performance of the energy detector significantly improved when the the number of cooperative users increases. The case with $L=1$ corresponds to the no-diversity scenario (single-relay system). The AWGN case is plotted for comparison purposes. AWGN channels are frequently assumed in the literature to investigate the energy detection performance. Clearly, such assumption over estimates the detection accuracy as can be seen from the plotted curves. To compare the performance of the diversity system between AF and DAF strategies, we plot in Fig. 4.3, $\bar{P}_{d}^{A F}$ and $\bar{P}_{d}^{D A F}$ for different number of cooperative users. It is obviously seen that the detection accuracy is greatly improved when the number of cooperative users increases for both strategies. Moreover, the DAF strategy performs better than the AF strategy for all the diversity scenarios. This can be explained by considering that only cooperative users with reliable detection probability are allowed to forward their local measurements to the fusion center. The difference in the performance of the DAF and AF strategies becomes more distinguishable as the number of cooperative users increases. Obviously, the system performance, deteriorates if the number of the heavily faded users becomes large enough to induce a missed detection in the final decision. In Fig. 4.4, the average detection probability is plotted for three Nakagami- $m$ fading scenarios with the case $m=1$ corresponds to Rayleigh fading. The high performance achieved with higher values of $m$ refers to the fact that the fading severity is inversely proportional with the fading parameter, $m$. Furthermore, the DAF strategy shows a better performance compared to the AF strategy, specifically at sever fading environments. Fig. 4.5 shows that the detection accuracy is significantly improved 


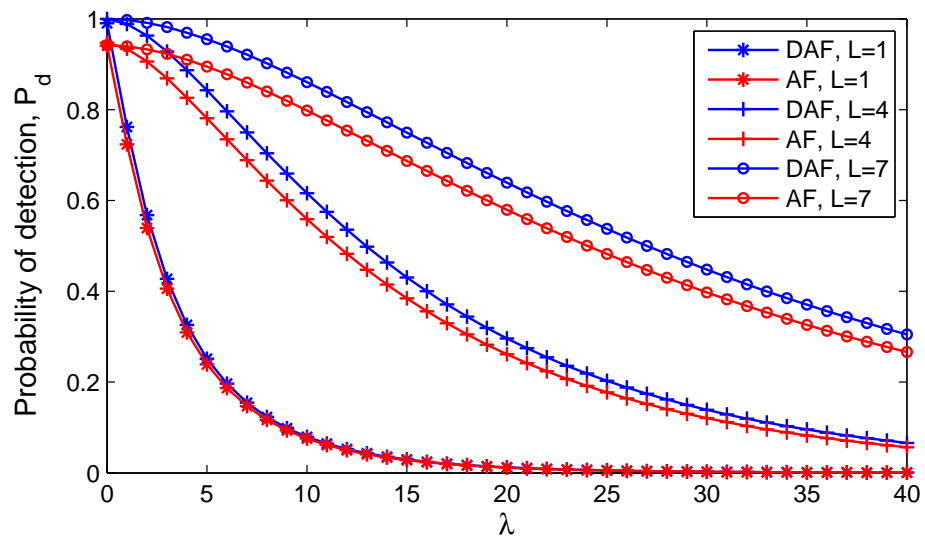

Figure 4.3: Detection performance of $\mathrm{AF}$ and DAF strategies for different diversity scenarios with $m=2, P=5 \mathrm{~dB}$, and $\bar{\gamma}_{s}=0 \mathrm{~dB}$.

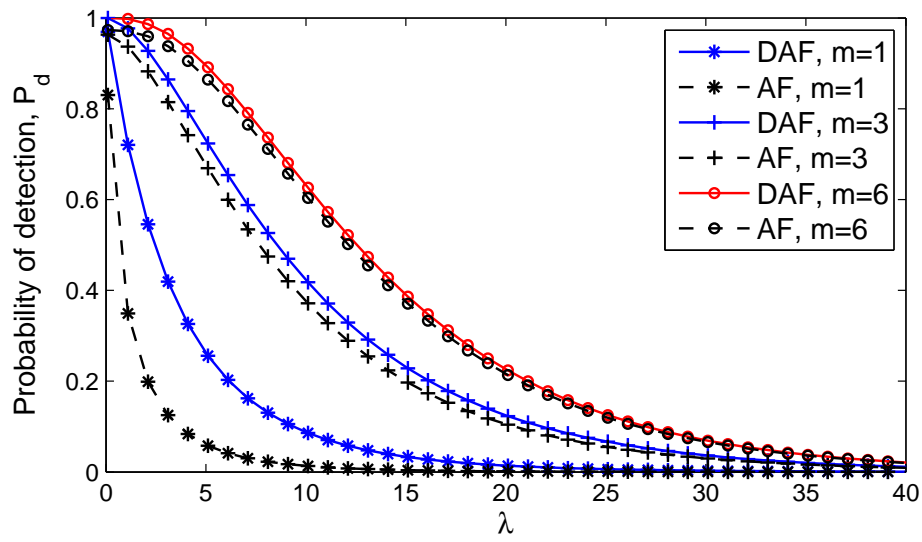

Figure 4.4: Detection performance of AF and DAF strategies for different fading scenarios with $L=3, P=5 \mathrm{~dB}$, and $\bar{\gamma}_{s}=0 \mathrm{~dB}$. 


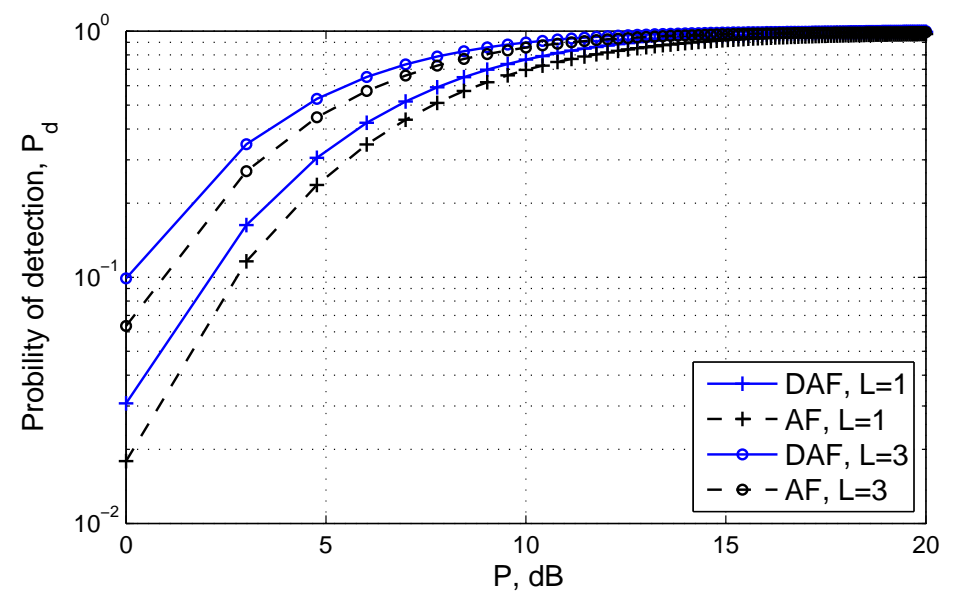

Figure 4.5: Detection performance of $\mathrm{AF}$ and DAF strategies for different power constraints with $\lambda=5, \mathrm{~m}=2$, and $\bar{\gamma}_{s}=0 \mathrm{~dB}$.

by increasing the relay power constraint, $P$. The DAF strategy shows a better performance compared to the AF strategy for all the selected values of the power constraint. Clearly, refraining the heavily faded relays improves the detection accuracy and explains the reason why DAF strategy outperforms the AF strategy in the all suggested scenarios. Yet, the AF performance becomes very close to the DAF performance at higher values of $P$. A target value of $\bar{P}_{d}>0.9$ is achieved with $P=10 \mathrm{~dB}$ for $L=3$ and with $P=13$ $\mathrm{dB}$ for $L=1$. This indicates, a gain of $3 \mathrm{~dB}$ can be achieved by switching from the a non-cooperative case to a cooperative case with $L=3$ users. Beyond the target value, the curves slow down as $\bar{P}_{d}$ approaches unity. Thus, there is no need to increase the transmission power to push $\bar{P}_{d}$ beyond this target value, since the higher transmission power leads to a higher interference level that may affect the operation of the primary network. 


\section{Chapter 5}

\section{End-to-End Performance over}

\section{Rayleigh Fading Channels}

\subsection{Introduction}

In this chapter, we incorporate sensing time and end-to-end channel statistics into the performance analysis of cooperative CR networks. First, a closed form expression for the PDF of the end-to-end SNR is obtained then, a closed-form expression for the average detection probability for high SNR applications is derived.

\subsection{Spectrum Sensing Model}

We assume that the sensing links as well as the reporting links are to be subjected to i.i.d Rayleigh fading and having identical average SNRs, which we denote as $\bar{\gamma}_{s}$ and 
$\bar{\gamma}_{r}$, respectively. The additive Gaussian noise of the sensing and relaying channels are are assumed to be i.i.d white Gaussian random processes with zero means and unit variances. Each CR acts as CSI-assisted relay and the fusion center is assumed to have a full knowledge of the CSI, i.e., it can acquire the global knowledge of sensing and relaying channel gains.

It is known that a sample function, of duration $T$, of a process which has a bandwidth $W$ is described by a set of sample values $2 T W$ in number which is assumed to be an integer for simplicity [81]. By means of a sampling plan, the energy of the signal sensed by the $i$ th $\mathrm{CR}$ denoted by $Y_{i}$ can be viewed as a sum of squares of statistically independent Gaussian random variables having zero means and unity variances [81]. It is then $Y_{i}$ follows a chi-square distribution with $2 T W$ degrees of freedom [30]. Therefore, over the $i$ th link, $P_{d_{i}}$ can be expressed as

$$
P_{d_{i}}=P\left\{Y_{i}>\lambda_{i} \mid H_{1}\right\}=Q_{u}\left(\sqrt{a \gamma_{e_{i}}}, \sqrt{\lambda_{i}}\right)
$$

where, $\gamma_{e_{i}}$ is the instantaneous end-to-end SNR, $u=2 T W$ is the degree of freedom assumed to be an integer number for simplicity, and $Q_{u}(.,$.$) denotes the generalized$ Marqum-Q function defined in [112, Eq.12] as $Q_{u}(\alpha, \beta)=\int_{\beta}^{\infty} x^{u} e^{-\frac{x^{2}+\alpha^{2}}{2}} I_{u-1}(\alpha x) \mathrm{dx}$, where $I_{u-1}($.$) denotes the modified Bessel function of (u-1)$ th order. 


\subsubsection{End-to-End Cooperative Spectrum Sensing Model}

A selection combining technique which selects the branch that has the maximum SNR among all the diversity branches using a simple receiver structure is implemented for the end-to-end performance. The average detection probability, $\bar{P}_{d}$, is evaluated over the distribution of the end-to-end SNR, $\gamma_{e}$. From (3.5), the end-to-end SNR of the $i$ th link, $\gamma_{e_{i}}$, under $H_{1}$ hypothesis can be expressed as

$$
\gamma_{e_{i}}=\frac{\left[h_{r_{i}} \sqrt{A_{i}}\right]^{2} E_{i}}{\left[\left(h_{r_{i}} \sqrt{A_{i}}\right)^{2}+1\right] u}
$$

where, the noise variance in (3.5), $N_{0}$, is assumed unity. Substituting Equation (3.6) in Equation (5.2), leads to $\gamma_{e_{i}}$ given by

$$
\gamma_{e_{i}}=\frac{\gamma_{s_{i}} \gamma_{r_{i}}}{\gamma_{s_{i}}+\gamma_{r_{i}}+1}
$$

where, $\gamma_{s_{i}}=E_{i} / u$ and $\gamma_{r_{i}}=h_{r_{i}}^{2} P_{i} / u$ are the per-hop SNR of the $i$ th sensing channel and the $i$ th reporting channel, respectively. The expression given in (5.3) is not easily tractable. Fortunately, it is found to be tightly bounded by [70]

$$
\gamma_{e_{i}}=\frac{\gamma_{s_{i}} \gamma_{r_{i}}}{\gamma_{s_{i}}+\gamma_{r_{i}}}
$$

which is mathematically tractable. Under fading conditions, the average detection probability, $\bar{P}_{d}$, is evaluated by averaging equation (5.1) over the distribution of the end-to-end 
$\mathrm{SNR}, f_{\Gamma_{e}}(\gamma)$

$$
\bar{P}_{d}=\int_{0}^{\infty} Q_{u}(\sqrt{a \gamma}, \sqrt{\lambda}) f_{\Gamma_{e}}(\gamma) \mathrm{d} \gamma
$$

Deriving the average detection probability is the most crucial part in this analysis for two reasons [113]: i) it requires one to obtain the end-to-end statistics of the dualhop relay-based cooperative system, and ii) it depends on the general methods used to evaluate integrals involving the generalized Marcum-Q function [114]. Over Rayleigh fading, the instantaneous SNR, $\gamma_{j}, j=s, r$ follows an exponential PDF given by

$$
f_{\Gamma_{j}}(\gamma)=\frac{1}{\bar{\gamma}_{j}} e^{-\frac{\gamma}{\bar{\gamma}_{j}}}, \quad \gamma \geq 0
$$

where, $\bar{\gamma}_{j}$ denotes the per-hop average SNR. It is obvious from (5.4) that $\gamma_{e_{i}}$ expression is related to a harmonic mean ${ }^{3}$ of two exponential random variables [70]. For a general case, we define the random variable $X_{j} \sim \varepsilon\left(\beta_{j}\right),(j=s, r)$, where the parameter $\beta_{j}=\frac{1}{\bar{\gamma}_{j}}$. The PDF of $X_{j}$ is already derived in $[70]$

$$
f_{X}(x)=\frac{1}{2} \beta_{s} \beta_{r} x e^{-\frac{x}{2\left(\beta_{s}+\beta_{r}\right)}}\left[\frac{\beta_{s}+\beta_{r}}{\sqrt{\beta_{s} \beta_{r}}} K_{1}\left(x \sqrt{\beta_{s} \beta_{r}}\right)+2 K_{0}\left(x \sqrt{\beta_{s} \beta_{r}}\right)\right] U(x)
$$

and the CDF is given by

$$
F_{X}(x)=1-\beta_{s} \beta_{r} x e^{-\frac{x}{2\left(\beta_{s}+\beta_{r}\right)}} K_{1}\left(x \sqrt{\beta_{s} \beta_{r}}\right)
$$

\footnotetext{
${ }^{3}$ The harmonic mean of two variables $X_{1}$ and $X_{2}$, is given by $X=m_{H}\left(X_{1}, X_{2}\right)=\frac{2 X_{1} X_{2}}{X_{1}+X_{2}}$
} 
where, $U($.$) is the unit step function, K_{0}($.$) is the zeroth-order modified Bessel function of$ the second kind defined in [106, Eq. 9.6.21], and $K_{1}($.$) is the first-order modified Bessel$ function of the second kind defined in [106, Eq. 9.6.22]. For L independent inputs, the output of the selection combining receiver is expressed as: $X_{\max }=\max \left(X_{1}, X_{2}, \cdots, X_{L}\right)$. Using the ordered statistics given in [115, Sec. 7.1], the PDF of the combined output can be written as

$$
\begin{aligned}
f_{X_{\max }}(x)= & \frac{L \beta_{s} \beta_{r}}{2}\left[\left[1-x \sqrt{\beta_{s} \beta_{r}} e^{-\frac{x}{2\left(\beta_{s}+\beta_{r}\right)}} K_{1}\left(x \sqrt{\beta_{s} \beta_{r}}\right)\right]^{L-1} x e^{-\frac{x}{2\left(\beta_{s}+\beta_{r}\right)}}\right. \\
& {\left.\left[\frac{\beta_{s}+\beta_{r}}{\sqrt{\beta_{s} \beta_{r}}} K_{1}\left(x \sqrt{\beta_{s} \beta_{r}}\right)+2 K_{0}\left(x \sqrt{\beta_{s} \beta_{r}}\right)\right]\right] . }
\end{aligned}
$$

Applying the Binomial theorem, equation (5.9) can be written as

$$
\begin{aligned}
f_{X_{\text {max }}}(x)= & \frac{L \beta_{s} \beta_{r}}{2}\left[\left[\sum_{k=0}^{L-1}\left(\begin{array}{c}
L-1 \\
k
\end{array}\right)(-1)^{k} e^{-\frac{k x}{2\left(\beta_{s}+\beta_{r}\right)}} x^{k}\left(\sqrt{\beta_{s} \beta_{r}}\right)^{k}\left(K_{1}\left(x \sqrt{\beta_{s} \beta_{r}}\right)\right)^{k}\right]\right. \\
& \left.x e^{-\frac{x}{2\left(\beta_{s}+\beta_{r}\right)}}\left[\frac{\beta_{s}+\beta_{r}}{\sqrt{\beta_{s} \beta_{r}}} K_{1}\left(x \sqrt{\beta_{s} \beta_{r}}\right)+2 K_{0}\left(x \sqrt{\beta_{s} \beta_{r}}\right)\right]\right] .
\end{aligned}
$$

Equation (5.10) is not easily tractable when it is required to evaluate integrals involving the generalized Marcum-Q function. Fortunately, the Bessel function $K_{1}(x \sqrt{\beta})$ appearing in $f_{X_{\max }}(x)$ can be approximated to a single polynomial term as the scale factor $\beta=\beta_{s} \beta_{r} \rightarrow 0^{+}$which is the case when the value of the average SNR is high. It has been shown that at high SNR applications, the system performance is determined 
by the behavior of $f_{X_{\max }}(\beta)$ close to $\beta=0$ [116]. In the following, we will employ this approximation to derive the PDF of the end-to-end SNR as well as the average detection probability of the proposed relay-based cooperative approach.

Theorem: For a high SNR system, the PDF of the combined signal is given by

$$
f_{\Gamma_{\max }}(\gamma)=\frac{L}{\bar{\gamma}_{s} \bar{\gamma}_{r}} \sum_{k=0}^{L-1}\left(\begin{array}{c}
L-1 \\
k
\end{array}\right)(-1)^{k} e^{-\frac{(k+1) \bar{\gamma}_{s} \bar{\gamma}_{r}}{\bar{\gamma}_{s}+\bar{\gamma}_{r}} \gamma}\left[\left(\bar{\gamma}_{s}+\bar{\gamma}_{r}\right)+4 \gamma K_{0}\left(\gamma \frac{2}{\sqrt{\bar{\gamma}_{s} \bar{\gamma}_{r}}}\right)\right](5
$$

Proof: See Appendix C.1.

Corollary 1: Based on equation (5.11), the average detection probability, $\bar{P}_{d}$, is given by,

$$
\begin{aligned}
\bar{P}_{d}= & \frac{L}{\bar{\gamma}_{s} \bar{\gamma}_{r}} \sum_{k=0}^{L-1}\left(\begin{array}{c}
L-1 \\
k
\end{array}\right)(-1)^{k}\left[\frac { 2 ( \overline { \gamma } _ { s } + \overline { \gamma } _ { r } ) } { p } e ^ { - \frac { \lambda } { 2 } } \left[\left(\frac{p+a}{a}\right)^{u-1}\left(e^{\frac{\lambda a}{2(p+a)}}-\sum_{n=0}^{u-2} \frac{1}{n !}\left(\frac{\lambda a}{2(p+a)}\right)^{n}\right)\right.\right. \\
& \left.+\sum_{n=0}^{u-2} \frac{1}{n !}\left(\frac{\lambda}{2}\right)^{n}\right]+\frac{4}{\Gamma(u)}\left(\frac{\sqrt{a}}{2}\right)^{u-1} \sum_{i=1}^{N} \frac{\Psi_{i}\left(t_{i}+\sqrt{\lambda}\right)^{u-1}}{c^{2}-d^{2}} \\
& {\left.\left[\frac{c}{\sqrt{c^{2}-d^{2}}} \ln \left(\frac{c}{d}+\sqrt{\left(\frac{c}{d}\right)^{2}-1}\right)-1\right]\right] }
\end{aligned}
$$

Proof: See Appendix C.2.

An efficient performance metric used to quantify the impact of fading is the Amount of Fading (AoF). AoF is useful in capturing the variations of the received SNR caused by the poor fading conditions. Since the diversity combining techniques are introduced to combat the SNR fluctuations, AoF is the appropriate performance measure to accurately reflect the behavior of the these techniques and quantify their detection reliability. AoF 
can be expressed in terms of the first and second moments of the end-to-end SNR, $\gamma_{e}$, as follows

$$
A o F=\frac{E\left(\gamma_{e}^{2}\right)-\left[E\left(\gamma_{e}\right)\right]^{2}}{\left[E\left(\gamma_{e}\right)\right]^{2}}
$$

where, $E($.$) denotes the expected value and the term E\left(\gamma_{e}^{2}\right)-\left[E\left(\gamma_{e}\right)\right]^{2}$ denotes the variance of $\gamma_{e}$.

Corollary 2: Based on equation (5.11), MGF of the end-to-end $\mathrm{SNR}, \mathcal{M}_{\Gamma}(s)=$ $E_{\Gamma}\left\{e^{-s \gamma}\right\}$, is given by

$$
\begin{aligned}
\mathcal{M}_{\Gamma}(s)= & L v^{2} \sum_{k=0}^{L-1}\left(\begin{array}{c}
L-1 \\
k
\end{array}\right)(-1)^{k}\left[\frac{1}{k+1+v s}+\frac{k+1+v s}{\left((k+1+v s)^{2}-d^{2} v^{2}\right)^{\frac{3}{2}}} \ln (\right. \\
& \left.\left.\frac{k+1+\sqrt{(k+1+v s)^{2}-d^{2} v^{2}}+v s}{d v}\right)-\frac{d^{2} v^{2}}{(k+1+v s)^{2}-d^{2} v^{2}}\right]
\end{aligned}
$$

where, $v=\frac{\bar{\gamma}_{s}+\bar{\gamma}_{r}}{\bar{\gamma}_{s} \bar{\gamma}_{r}}$ and $d=\frac{2}{\sqrt{\bar{\gamma}_{s} \bar{\gamma}_{r}}}$.

Proof: The integral involved in the derivation of the MGF can be evaluated with the help of [105, Eq. 6.624.1], which once used with some algebra leads to the desired result in (5.14).

The $n$th moment of $\gamma_{e}$ is given by

$$
E\left(\gamma_{e}^{n}\right)=\left.\frac{\mathrm{d}^{n}}{\mathrm{ds}^{n}} \mathcal{M}(s)\right|_{s=0}
$$

therefore, the first and second moments of $\gamma_{e}$ are obtained by evaluating the first and 
second derivatives of (5.14) at $s=0$ as follow

$$
\begin{aligned}
E\left(\gamma_{e}\right)= & L v^{3} \sum_{k=0}^{L-1}\left(\begin{array}{c}
L-1 \\
k
\end{array}\right)(-1)^{k}\left[\frac{3 d^{2}(k+1)}{(k+1)^{2}-d^{2} v^{2}}-\frac{d^{2}\left(2(k+1)^{2}+d^{2} v^{2}\right)}{\left((k+1)^{2}-d^{2} v^{2}\right)^{\frac{5}{2}}} \ln (\right. \\
& \left.\left.\frac{k+1+\sqrt{(k+1)^{2}-d^{2} v^{2}}}{d v}\right)-\frac{1}{(k+1)^{2}}\right] \\
E\left(\gamma_{e}^{2}\right)= & L v^{4} \sum_{k=0}^{L-1}\left(\begin{array}{c}
L-1 \\
k
\end{array}\right)(-1)^{k}\left[\frac{2}{(k+1)^{3}}+\frac{3 d^{2}\left(2(k+1)^{3}+3 d^{2} v^{2}(k+1)\right)}{\left((k+1)^{2}-d^{2} v^{2}\right)^{\frac{7}{2}}} \ln (\right. \\
& \left.\left.\frac{k+1+\sqrt{(k+1)^{2}-d^{2} v^{2}}}{d v}\right)-\frac{d^{2}\left(11(k+1)^{2}+4 d^{2} v^{2}\right)}{(k+1)^{2}-d^{2} v^{2}}\right]
\end{aligned}
$$

From (5.16) and (5.17), we can obtain the AoF for the proposed cooperative diversity approach.

\subsection{Performance Evaluation}

For evaluation purposes, the modulation parameter $a$ is set to a value of 2 , the sampling rate of the local sensing is set to a value of $1 \mathrm{MSa} / \mathrm{s}$, and $E_{i}$ is set to a unity (0 $\mathrm{dB})$. To validate the accuracy of the derived equation, the closed-form expression of the average detection probability derived in (5.12) has been numerically evaluated through comparison with Monte Carlo simulation results obtained over 100,000 iterations as shown in Fig. 5.1. It is obvious from this figure that the analytical results extremely match the simulated ones as $\lambda$ increases. A detection accuracy up to 4 decimal points 


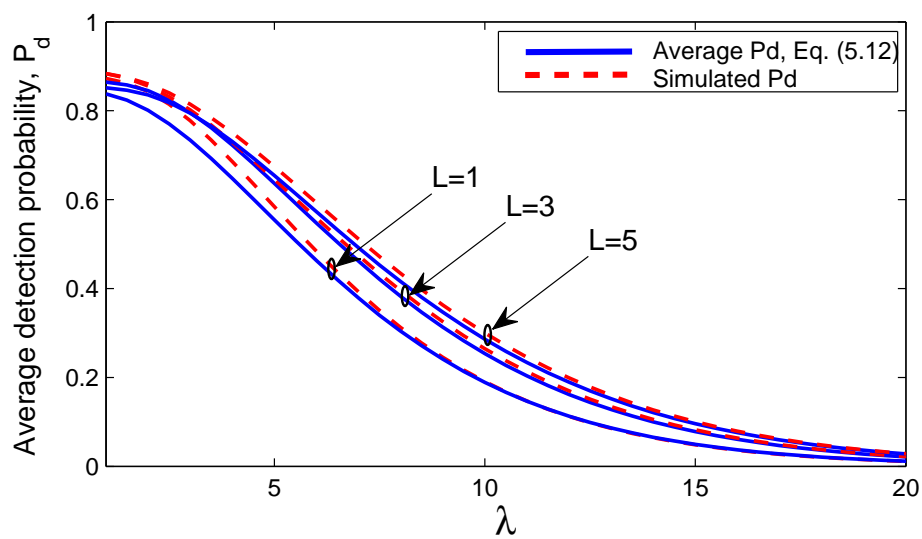

Figure 5.1: Comparison of average detection probability in equation (5.12) with Monte Carlo simulation for $\mathrm{L}=1,2$, and 3. $\bar{\gamma}_{s}=\bar{\gamma}_{r}=5 \mathrm{~dB}, u=6$.

is achieved with $\lambda>5$. However, as $\lambda \rightarrow 0^{+}$, the theoretical results diverge more from the simulated ones and the derived closed-form expression given in (5.12) underestimates the average detection probability. For such extremely low values of $\lambda$, the numerical integration techniques can be used to directly evaluate the expression given in equation (C.4). The curves mismatch at very low values of the decision threshold can be explained by considering the Gaussian-Laguerre integration used for the generalized Marcum-Q function approximation. In Fig. 5.2 we plot the generalized Marcum-Q function directly computed using a MATHMETICA-8 function with the approximated expression form given in (C.6). From this figure, one can easily recognize how the two curves diverge as $\lambda \rightarrow 0^{+}$. Recall that $\lambda^{\prime}$ 's value is not related to the SNR but associated to the targeted $P_{f}$ which may vary from one application to another. In Fig. 5.3, we plot the average detection probability versus the number of energy samples collected during the sensing time window. The number of collected samples is varied from 1 to 10 samples. For a sampling rate of $1 \mathrm{MSa} / \mathrm{s}$, this corresponds to a sensing window of 1 to $10 \mu \mathrm{s}$. 


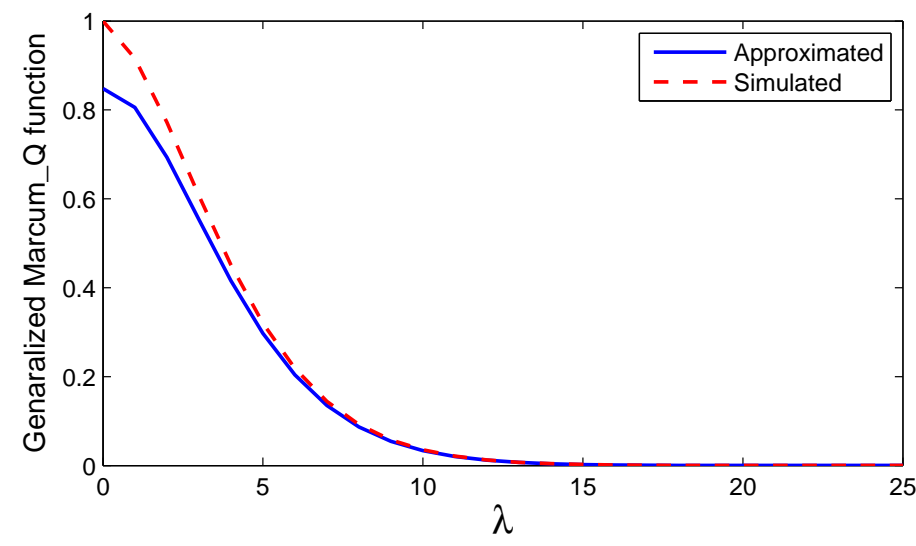

Figure 5.2: Comparison of approximated and exact Marcum_Q function. $u=6$.

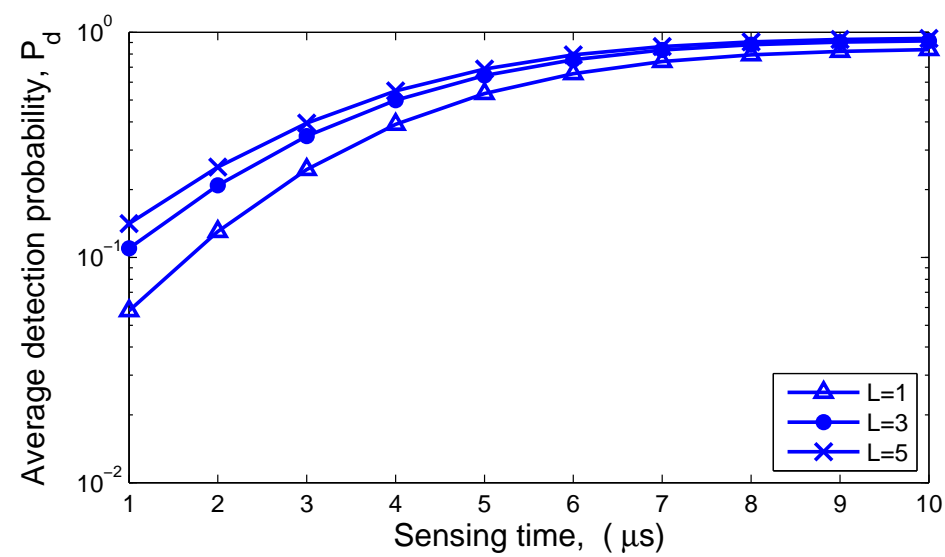

Figure 5.3: Average detection probability $\bar{P}_{d}$ vs. sensing Time for $\mathrm{L}=1,3$, and $5 . \bar{\gamma}_{s}=$ $\bar{\gamma}_{r}=5 d B$. 
The plotted curves demonstrate that the cooperative diversity gain is achieved over all the selected sensing windows. The most interesting observation is that the detection probability slightly increases after a certain sensing time window. Therefore, there is no need to prolong the sensing time which would affect the spectrum sensing efficiency. Recall that a high sensing efficiency means efficient bandwidth usage and more spectrum access opportunities for secondary users and a higher CR network throughput. The plotted results indicate that a targeted detection probability of 0.9 is achieved with a time window of $8 \mu s$ for the diversity case with $L=5$ and $9 \mu s$ for the diversity case with $L=3$.

To demonstrate the importance of including the reporting channel conditions for spectrum sensing system evaluations, we plot in Fig. 5.4 the average detection probability versus the reporting channel average SNR. The sensing time window is set to a value of $8 \mu \mathrm{s}$. As shown, $\bar{P}_{d}=0.9$ is achieved for a diversity system with 5 cooperative users at $\bar{\gamma}_{r}=5 d B$. The average detection probability starts to slow down beyond this value of $\bar{\gamma}_{r}$ as it slowly increases close to $\bar{P}_{d}=1$. Clearly, there is no need to increase the transmission power to higher than this targeted value since the detection probability increases a little even when $\bar{\gamma}_{r}$ becomes $10 \mathrm{~dB}$ and $15 \mathrm{~dB}$. Remember that, higher transmission power increases the the average false alarm probability and leads to a higher interference level that may affect the primary network operation. In Fig. 5.5, we plot the AoF versus the number of cooperative users, L, for three different values of the reporting channel average SNR. For comparison purposes, the AoF is normalized 


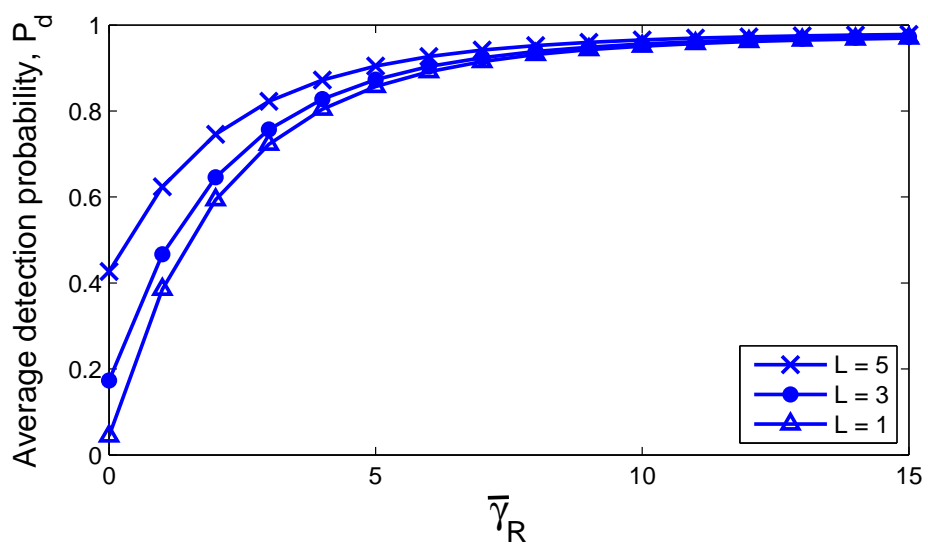

Figure 5.4: Average detection probability, $\bar{P}_{d}$, vs. $\bar{\gamma}_{r}$, for $\mathrm{L}=1$, 3, and $5 . \bar{\gamma}_{s}=5 d B$, $u=8$.

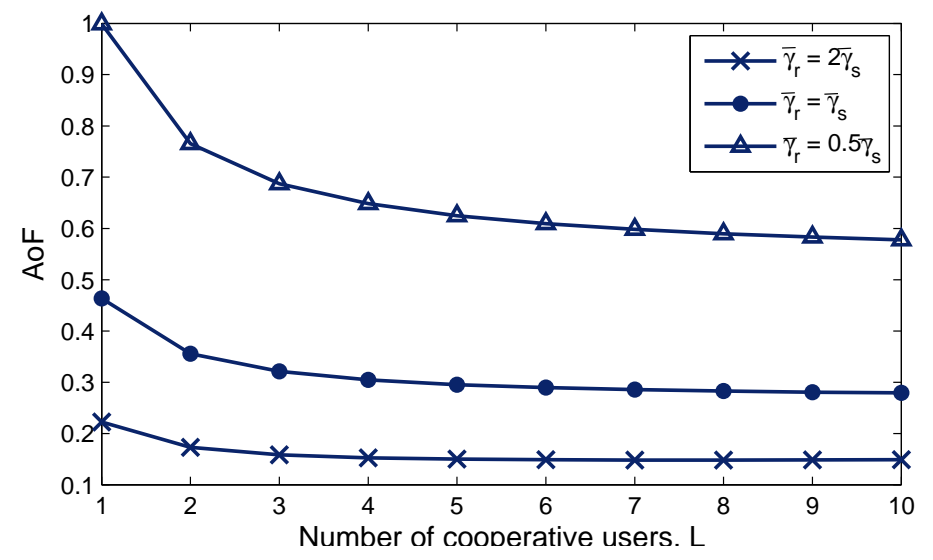

Figure 5.5: Amount of fading vs. number of cooperative users. $\bar{\gamma}_{s}=10 \mathrm{~dB}$. 
with respect to its maximum obtained value. Clearly, the cooperative selection diversity effectively enhances the receiver performance by reducing the AoF associated to the end-to-end SNR. A remarkable reduction in AoF can be seen once we move from the no diversity case with $\mathrm{L}=1$ to the first diversity case with $\mathrm{L}=2$. The AoF reduction rate then starts to slow down as L increases. The figure also shines some light on the power balance between the sensing and the reporting channels. For the best detection reliability, the reporting channel average SNR should be made greater than that of the sensing channel to minimize the fluctuations of the end-to-end SNR. 


\section{Chapter 6}

\section{Cluster-Based Cooperative}

\section{Spectrum Sensing}

\subsection{Introduction}

In this chapter, we propose a cluster-based spectrum sensing approach that reduces the bandwidth requirements for reporting data to the fusion center by reducing the number of reporting terminals to a minimal reporting set. The approach replaces the secondary base station by a local fusion center to mitigate the destructive channel conditions of global relaying channels. We also propose a new approach to select the location of the fusion center based on the general center scheme in graph theory. We assume one of the elected cluster heads acts as a center entity and serves as a master fusion center to finalize the cooperative decision and broadcasts it back to other cluster heads and 
from there to cluster members. The minimal dominating set (MDS) clustering approach is used to obtain the minimal set of clusters that keep the network connected. The influence of cluster size, number of clusters, and relaying channels probability of error on the cognitive radio network performance is investigated. The results obtained reveal that cluster-based CSS systems outperform conventional CSS systems in terms of throughput capacity especially when the relaying channels are subjected to high probability of error.

\subsection{Network Model}

We assume that $L$ cognitive radio terminals are distributed in a square field of area $A$ as a two-dimensional Poisson point process with a density $\mu$. A node $v$ (CR user) is said to be in a neighborhood of a node $u$, if $v$ is within a distance at most $r_{c}$ from $u$, where $r_{c}$ is the cluster radius. Each CR terminal is identified by a unique ID and the CR users are assumed static or moving slowly during the algorithm execution.

The topology of the CR network is presented by an undirected graph $G(V, \Xi)$, where $V$ is a set of vertices in this graph that stands for CR terminals and $\Xi$ is a set of links between those terminals. A link $(u, v) \in \Xi$ means that terminals $u$ and $v$ share at least one common channel and in 1-hop distance from each other. The neighborhood set of a given node $v \in V$, represents all the nodes in a 1-hop away from $v$ that share at least one channel with $v$.

Transmission power determines the cluster radius and largely affects the number of clusters, cluster size, and network performance. Higher transmission power means, fewer 
hops resulting in higher network throughput. However, the higher interference resulting from higher transmission power tends to limit the network throughput. This is true in the other way as well.

This study investigates the impact of transmission power on the network topology. Precisely, we investigate how the number of isolated nodes $\left(N_{i s o}\right)$ is changing with cluster radius. Isolated nodes refer to those nodes that can't find any neighboring terminal within a radius of $r_{c}$ in any channel within their channel sets. These, nodes declare themselves as cluster heads and form their own clusters (single-node clusters).

Since CR users are distributed as a point Poisson process with a density $\mu$, then, the probability that a node is unable to find any neighboring node within a radius $r_{c}$ is $e^{-\mu \pi r_{c}^{2}}$. If we consider a two dimensional polar coordinate $(r, \theta)$, where the node is in the origin, then the average of isolated node $E\left\{N_{\text {iso }}\right\}$ can be calculated as follows,

$$
\begin{aligned}
E\left\{N_{i s o}\right\} & =\mu A \int_{0}^{2 \pi} \int_{h_{p} r_{c}}^{\infty} e^{-\mu \pi\left(h_{p} r\right)^{2}} \mu r \mathrm{~d} r \mathrm{~d} \theta \\
& =\mu A e^{-\mu \pi\left(h_{p} r_{c}\right)^{2}}
\end{aligned}
$$

where, $h_{p}$ denotes the number of transmission hops. Since our approach is to establish a connected minimal dominating set of connected clusters that covers all the deployed CR terminals, then the fraction of single-node clusters, $E\left\{N_{i s o}\right\}$, must be very small. If $\kappa,(\kappa>0)$, is an arbitrarily small number that represents a target percentage of singlenode clusters such that, 


$$
E\left(N_{i s o}\right) \leq \kappa \mu A .
$$

then by equating (6.1) and (6.2) we obtain the following lower bound of cluster radius at this specified target,

$$
r_{c} \geq \sqrt{\frac{-\log \kappa}{\pi \mu h_{p}^{2}}} .
$$

Increasing the cluster radius will clearly increase the probability to find a neighboring terminal for each node and increase the cluster size. However, as the cluster size increases, the probability to have network bottlenecks at the inter-cluster communication gateways increases. Also, the inter-cluster and intra-cluster interferences tend to increase with larger cluster communication range. Hence, to avoid congestion at those nodes, we introduce an upper bound for the cluster size such that the traffic load at those gateways will be upper bounded and adhere with the QoS requirements.

With low cross correlation CDMA codes, inter-cluster interference can be eliminated. We assume each cluster is assigned a unique transmitting code that is different from those codes used in neighbor clusters. Since the receiver nodes must be set to the same code as the designated transmitter, interference with other clusters is avoided. If no two nodes in a cluster are transmitting simultaneously, there will be no intra-cluster interference. Following [117], we assume that within each cluster, an appropriate scheduling scheme is available to facilitate the transmission of cluster members. 


\subsection{The Clustering Algorithm}

In graph theory [90], the minimal dominating set problem is to find a subset, $\mathcal{C}(|\mathcal{C}|=M)$ called the dominating set such that each node belongs to one member of $\mathcal{C}$. We refer to the dominating set members as cluster heads and the nodes that belong to one of the cluster heads as cluster members.

The algorithm requires a preliminary node discovery and a master channel configuration phase. The master channel is used by the cluster heads and cluster members to exchange the control data necessary for the cluster formation. During the preliminary node discovery each node exchanges neighbor information with the detected neighbor. We assume that at the completion of the node discovery, any node say $v_{i}$ knows the channel availability set of node $v_{j} \in v_{i}^{1}$ and the set of 1-hop neighbors of the node $v_{j}, \forall v_{j} \in v_{i}^{1}$. Where $v_{i}^{1}$ denotes the 1-hop neighborhood of node $v_{i}$. The preliminary node discovery is followed by initial cluster setup phase. In this phase, each node orders its channels with frequency and starts from the lowest one to listen for beacons from other nodes. If no message comes in the listening interval, the node forms a cluster on the listening channel and becomes the cluster head. if a beacon comes on the listening interval, the node requests to join the cluster through the initial cluster setup phase. A cluster head discovers its $k$-hop neighbor clusters from the collected neighbor information and makes interconnection by choosing gateway nodes. The initial cluster setup stops when all nodes join clusters and clusters form interconnection.

The MDS cluster setup scheme is performed after the initial setup phase. Since, the 
network topology needs to be optimized from time to time as the network conditions changes, the MDS scheme is required to be performed periodically. Typically network topology changes when a primary user appears at a certain channel, a new terminal joins/leaves the network, or a cluster head reporting channel comes under a deep shadowing/fading.

In MDS scheme, every node $i \in V$ is required to be covered by one member of the dominating set $\mathcal{C} \subseteq V$. The dominating set contains $M$ subsets $\mathcal{C}_{1}, \mathcal{C}_{2}, \cdots, \mathcal{C}_{M}$ of the base set $V=\{1,2, \cdots, L\}$ such that $\cup_{j=1}^{M} \mathcal{C}_{j}=V$. We define a binary variable $x_{j}$ for the subsets $\mathcal{C}_{j}, j=1,2, \ldots, M$ as follows,

$$
x_{i}=\left\{\begin{array}{cc}
1 & \text { if } \mathcal{C}_{j} \in \mathcal{C} \\
0 & \text { otherwise }
\end{array}\right.
$$

By defining $a_{i j}$ to be 1 when a node $i \in \mathcal{C}_{j}$ and 0 otherwise, we can write the problem as,

$$
\min \sum_{\mathcal{C}_{j} \in \mathcal{C}} x_{j} \quad j=1,2, \ldots, M
$$

subject to,

$$
\begin{gathered}
\sum_{j \in \mathcal{C}} a_{i j}=1, \quad \forall i \in V \\
\sum_{j \in \mathcal{C}} a_{i j} d_{i j} \leq h_{p} r_{c}, \quad \forall i \in V \\
\sum_{i \in V} a_{i j} \leq \Lambda, \quad \forall j \in \mathcal{C}
\end{gathered}
$$


where, $\Lambda$ is an upper bound of cluster size.

We extend the single-hop CogMesh algorithm presented in [44] to a d-hop clustering scheme and generalized this algorithm for the above described system model. The MDS algorithm is performed at the node level to minimize the number of clusters in the neighborhood of a selected node and reconfigure the whole cluster topology when a new cluster set smaller than the original one can be found. Instead of selecting a node in a random way as in [44], our approach selects a node with the highest residual energy from a set of neighboring nodes that share a channel with a maximum degree to be a cluster head. The algorithm starts forming a cluster from this cluster head and all nodes within d-hop, $d=1,2, \ldots, h_{p}$, that shares the same channel. The new cluster head and all the assigned cluster members will be eliminated from the node neighborhood set. Then, a node with a maximum degree on another channel will be selected as a cluster head and its assigned cluster members eliminated too from the remaining node set and so on until all the nodes are configured in the new cluster topology. The algorithm then starts the gateway nodes selection to construct the inter-cluster communications. A priority case is used in gateways selection. A node in the shortest path between any two cluster heads is given the highest priority. Once a cluster is formed, the cluster head communicates with the neighbors to select the CDMA codes. Only when the code assignment is completed data can be transmitted in the network.

Lemma 1: In a d-hop cluster, the distance between any two nodes is at most $2 d r_{c}$ Proof: Let $v$ be any node belongs to cluster $\mathcal{C}_{j}, j=1,2, \ldots ., M$ whose cluster head 
is $m_{j}$. If $v(I D) \neq m_{j}(I D)$ then there must be another node $u \in \mathcal{C}_{j}$ at most in a d-hop away from $v$ where $u(I D)=m_{j}(I D)$. Hence, $u$ is the cluster head of the cluster $\mathcal{C}_{j}$ and $|v-u| \leq d r_{c}$. For any other node say $w \in \mathcal{C}_{j}$, whose $w(I D) \neq m_{j}(I D),|w-u| \leq d r_{c}$. Therefore, the distance between $v$ and $w$ is at most $2 d r_{c}$

Lemma 2: If $\mathcal{C}$ is a connected dominating set, then any cluster head is at most $(2 d+1) r_{c}$ away from the nearest cluster head

Proof: Let assume the contrary, and assume that the closest routing path between cluster heads $m_{1} \in \mathcal{C}_{1}$ and $m_{2} \in \mathcal{C}_{2}$ is $(2 d+2) r_{c}$. Let this closest path passes through the two gateways, $x$ and $y$, see Fig. 6.1. Since, $x \in \mathcal{C}_{1}$ and $y \in \mathcal{C}_{2}$, then $\left|m_{1}-x\right| \leq d r_{c}$ and $\left|m_{2}-y\right| \leq d r_{c}$ and $|x-y| \geq(2 d+2) r_{c}-2 d r_{c}=2 r_{c}$. This implies that each of $x$ and $y$ is not in a 1-hop transmission range of each other and the two clusters are not connected. Since the dominating set is a connected dominating set, then, there must be another gateway $v \in \mathcal{C}_{1}$ which is in 1-hop to another cluster head say, $m_{3} \in \mathcal{C}_{3}$ or adjacent node say $z \in \mathcal{C}_{3}$ which is at most d-hop from $m_{3}$, i.e., $\left|m_{3}-z\right| \leq d r_{c}$. Therefore, $\left|m_{1}-m_{3}\right|=\left|m_{1}-v\right|+|v-z|+\left|z-m_{3}\right| \leq d r_{c}+r_{c}+d r_{c}=(2 d+1) r_{c}$ and $m_{3}$ is the nearest cluster head to $m_{1}$.

The master fusion center is then elected from the minimal cluster head set. The placement of the fusion center also needs to be optimized. The fusion center placement problem is concerned with selecting the best location in a specified region for the network center entity. Mainly, there are two options to solve this location problem, the center problem and the general center [118]. In graph theory, the graph center is any vertex 


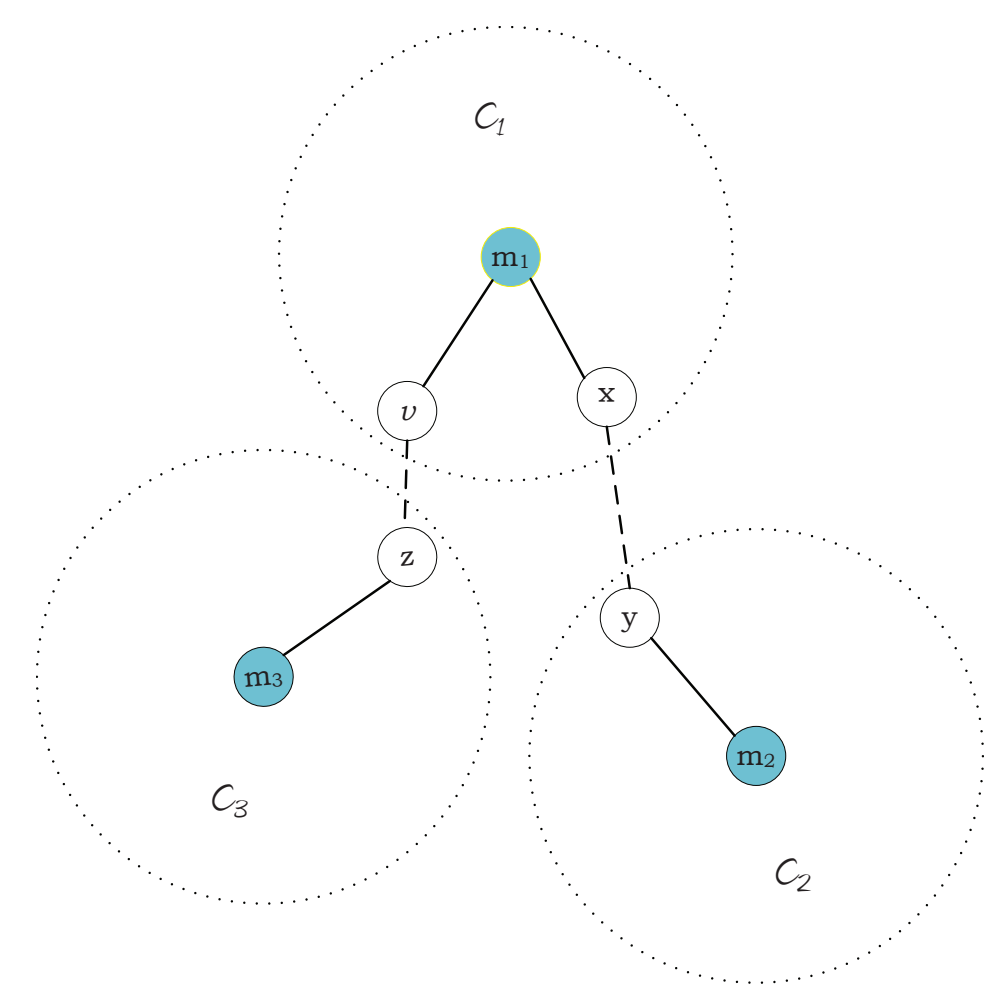

Figure 6.1: Maximum distance to the nearest cluster head

$v$ whose furthest vertex is as close as possible while the general center is any vertex $v$ where the aggregated distance from all other vertices is as minimum as possible. Let,

$$
\operatorname{Mvv}(i)=\max _{\substack{i \\ j \neq i}} d_{i j}
$$

denotes the maximum distance of any vertex from vertex $(i)$, where $d_{i j}$, is the vertex-tovertex distance, and,

$$
S v v(i)=\sum_{\substack{i \\ j \neq i}} d i j
$$

denotes the aggregated distance of all vertices from vertex $i$. 
Then the graph center is any vertex $x$ such that,

$$
\operatorname{Mvv}(x)=\min _{i}\{M v v(i)\}
$$

while the general center is any vertex $x$ with the smallest possible $S v v(i)$, i.e., the smallest aggregated distances from all other vertices or,

$$
\operatorname{Svv}(x)=\min _{i}\{\operatorname{Svv}(i)\}
$$

Practically, the reporting paths are subjected to different channel conditions. Therefore, the shortest reporting channels may not always be the best choice especially if they come under a deep fading/shadowing. In this work, we proposed a modified general center scheme that considers the channel gain of the $M-1$ signal paths between the fusion center and the $M-1$ cluster heads. In the proposed scheme, the fusion center is the cluster head whose aggregated channel gain has the maximum possible value. Since, the cooperative decision is made by combined received signals from different cluster heads, we believe that using the channel gain model in finding the best location of the fusion center is more accurate than considering merely the topological distances. Moreover, the channel gain is affected directly by both the transmitter-receiver distance and the random influence of fading and shadowing conditions. 6.2 shows a cluster-based cooperative spectrum sensing where one cluster head acts as a local fusion center.

Let $G_{i j}$ denotes the channel gain between a transmitter, $i$, and a receiver, $j$, then the 


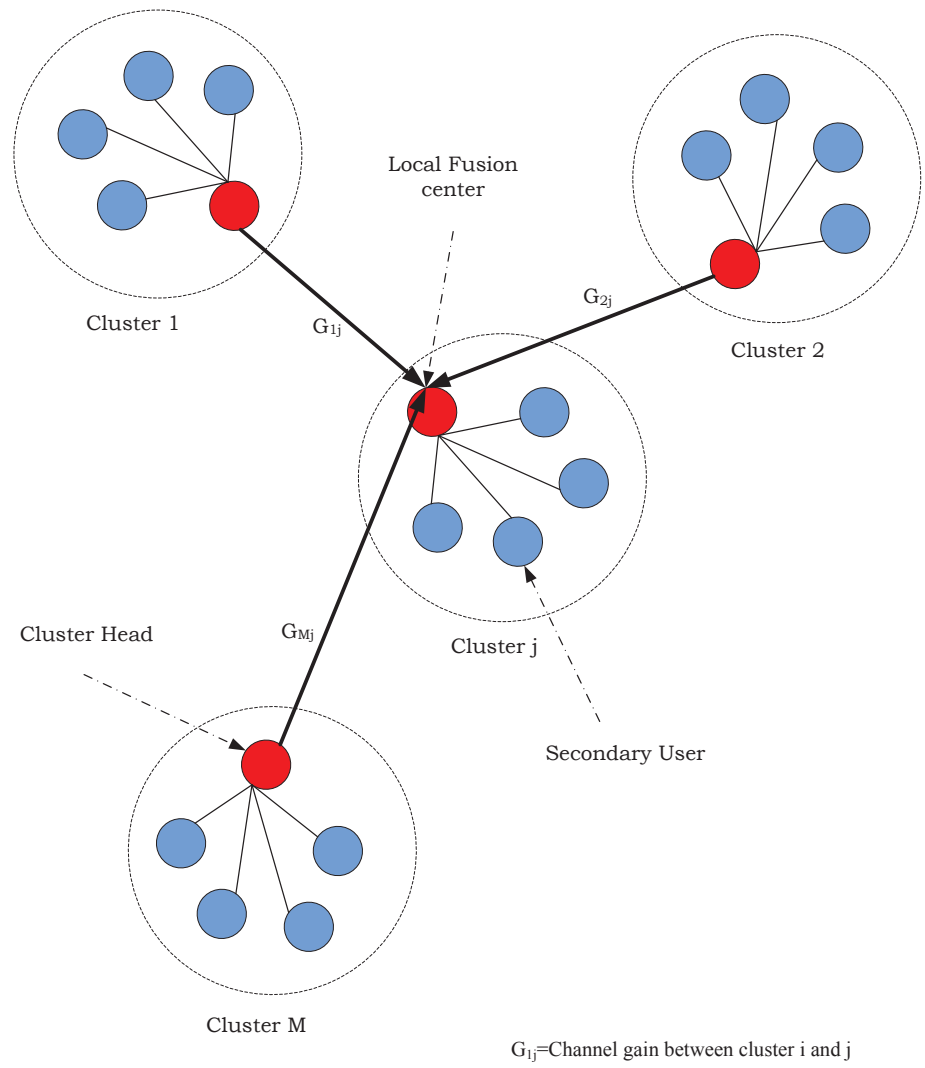

Figure 6.2: Cluster-based cooperative spectrum sensing 
master fusion center is the cluster head $m$ that has the maximum aggregate channel gain or,

$$
G h h(m)=\max _{i}\{G h h(i)\} \quad i=1,2, \ldots, M
$$

where,

$$
G h h(i)=\sum_{j} G_{i j} \quad j=1,2, \ldots, M
$$

\subsection{Cluster-Based Cooperative Spectrum Sensing}

The propose network scheme has two levels of data fusion. The first data fusion is performed at the cluster level by each cluster head and the second one is performed at the master fusion center. The channels are assumed to be subjected to i.i.d Rayleigh fading. The sensing information is shared only at the local scale within each cluster and there is no need for each CR terminal to send its own decision to the master fusion center. After receiving the local observations from all CR users within its cluster, the cluster head employs a selection combining scheme to make the cluster decisions.

Since the clusters are formed by a neighboring CR users, we assume the relaying links between cluster members and the cluster head as error-free channels. Accordingly, the probability of false alarm computed at the cluster level, $P_{f \mathcal{C}}^{j}$, will be independent of channel statistics. On the other hand, the probability of detection, $P_{d \mathcal{C}}^{j}$, will be determined by the fading conditions and the combining scheme employed by the cluster head. 
Therefore, $P_{f \mathcal{C}}^{j}$ and $P_{d \mathcal{C}}^{j}$ are given by [30]

$$
\begin{gathered}
P_{f \mathcal{C}}^{j}=\frac{\Gamma\left(u, \frac{\lambda_{C}}{2}\right)}{\Gamma(u)} \\
P_{d \mathcal{C}}^{j}=M \sum_{i=0}^{M-1} \frac{(-1)^{i}}{i+1}\left(\begin{array}{c}
M-1 \\
i
\end{array}\right) P_{d_{R a y}}\left(\frac{\bar{\gamma}_{s_{i}}}{i+1}\right)
\end{gathered}
$$

where,

$$
P_{d_{\text {Ray }}}\left(\bar{\gamma}_{s_{i}}\right)=e^{-\frac{\lambda_{C}}{2}} \sum_{n=0}^{u-2} \frac{1}{n !}\left(\frac{\lambda_{C}}{2}\right)^{n}+\left(\frac{1+\bar{\gamma}_{s_{i}}}{\bar{\gamma}_{s_{i}}}\right)^{u-1}\left[e^{-\frac{\lambda_{C}}{2\left(1+\bar{\gamma}_{s_{i}}\right)}}-e^{-\frac{\lambda_{C}}{2}} \sum_{n=0}^{u-2} \frac{1}{n !} \frac{\lambda_{C} \bar{\gamma}_{s_{i}}}{2\left(1+\bar{\gamma}_{s_{i}}\right)}\right]
$$

with each $\bar{\gamma}_{s_{i}}$ is replaced by $\frac{\bar{\gamma}_{s_{i}}}{i+1}$.

Equation (6.8), clearly illustrates that the spectrum sensing performance of each cluster is largely effected by the number of cluster size, the threshold $\lambda_{c}$, and the number of samples $u$. Under the bandwidth constraints of the reporting channel, we allow each cluster head to send only 1-bit decision $\{0\}$ for $H_{0}$ and $\{1\}$ for $H_{1}$ to the master fusion center rather than their decision statistics. The fusion center then makes the final decision according to the fusion rule implemented. The OR-rule is implemented in the proposed scheme. For the OR-rule, a decision of $\{0\}$ for $H_{0}$ is only made when all the $M$ cluster decisions demonstrate the absence of the primary user. Such kind of rule is perfect for interference avoidance to the primary user since the secondary user will only be allowed to access the spectrum if all the cluster heads reported the binary decision $\{0\}$ to the 
fusion center.

The decision rule at the master fusion center can be defined as:

$$
D= \begin{cases}1 & \sum_{j=1}^{M} \mathcal{B}_{j} \geq 1 \\ 0 & \text { otherwise }\end{cases}
$$

where, $\mathcal{B}_{j}, j=1, \cdots, M$ is the decision of the $j$ th cluster head. Therefore, under the OR-rule, the false alarm probability and the detection probability can be defined as:

$$
\begin{aligned}
& P_{f}=1-\prod_{j=1}^{M}\left(1-P_{f \mathcal{C}}^{j}\right) \\
& P_{d}=1-\prod_{j=1}^{M}\left(1-P_{d \mathcal{C}}^{j}\right) .
\end{aligned}
$$

Over faded channels, a reported decision of $\{0\}$ for primary network absence may be received at the fusion center as $\{1\}$, or a reported decision of $\{1\}$ for active primary network may be received at the fusion center as $\{0\}$. In the first case, a false alarm will be triggered, and a missed detection risk may be encountered in the second case. If $\widehat{P}_{f \mathcal{C}}^{j}$ denotes the probability of receiving $\{1\}$ at the master fusion center when the $j$ th cluster head reports $\{0\}$ and $\widehat{P}_{m \mathcal{C}}^{j}$ denotes the probability of receiving $\{0\}$ at the master fusion center when the $j$ th cluster head reports $\{1\}$, then under OR-rule, the probabilities of 
false alarm, $P_{f}$, and missed detection, $P_{m}$, become [53],

$$
\begin{gathered}
P_{f}=1-\prod_{j=1}^{M}\left[\left(1-P_{f \mathcal{C}}^{j}\right)\left(1-\widehat{P}_{f \mathcal{C}}^{j}\right)+P_{f \mathcal{C}}^{j} \widehat{P}_{m \mathcal{C}}^{j}\right] \\
P_{m}=\prod_{j=1}^{M}\left[P_{m \mathcal{C}}^{j}\left(1-\widehat{P}_{f \mathcal{C}}^{j}\right)+\left(1-P_{m \mathcal{C}}^{j}\right) \widehat{P}_{m \mathcal{C}}^{j}\right] .
\end{gathered}
$$

Let $P_{e}^{j}=\widehat{P}_{f \mathcal{C}}^{j}=\widehat{P}_{m \mathcal{C}}^{j}$, where $P_{e}^{j}$ denotes the probability of the reporting errors for cluster head $m_{j}$, then equations (6.12) and (6.13), can be rewritten as,

$$
\begin{gathered}
P_{f}=1-\prod_{j=1}^{M}\left[\left(1-P_{f \mathcal{C}}^{j}\right)\left(1-P_{e}^{j}\right)+P_{f \mathcal{C}}^{j} P_{e}^{j}\right] \\
P_{m}=\prod_{j=1}^{M}\left[P_{m \mathcal{C}}^{j}\left(1-P_{e}^{j}\right)+\left(1-P_{m \mathcal{C}}^{j}\right) P_{e}^{j}\right] .
\end{gathered}
$$

\subsection{Throughput Performance}

To meet the throughput requirements, the secondary users network needs to increase the sensing efficiency by decreasing the false alarm probability and increasing spectrum access opportunities. Since CR users can not transmit and sense at the same time, periodic sensing rounds are required where sensing and transmitting processes are alternating in a periodic manner in successive frames. The frame time, $T$, consists mainly of the sensing time $T_{s}$ and the transmission time $T_{D}$. We will ignore the clustering time as it is very small compared to the sensing and transmission time [38, 119]. For interference 
avoidance, the observation time needs to be long enough to achieve sufficient detection accuracy. But, for a fixed frame size $T=T_{s}+T_{D}$, increasing sensing time inevitably decreases the transmission time and consequently decreases sensing efficiency. On the other hand, a longer transmission time enhances the sensing efficiency but causes higher interferences to primary user as the detection accuracy decreases due to the lack of sufficient sensing information [13]. Let $\eta$ denotes the spectrum sensing efficiency, then:

$$
\eta=\frac{T_{D}}{T}=\frac{T_{D}}{T_{D}+T_{S}}
$$

The throughput of the secondary network comes as a result of the following two network operation cases [48]: (1) CR terminals communications and exchange data in the absence of the primary user with probability of $\left(1-P_{f}\right) P\left(H_{0}\right)$, (2) CR terminals communications when the primary user is mis detected by the secondary network with a probability of $\left(1-P_{d}\right) P\left(H_{1}\right)$ where, $P\left(H_{0}\right)$ and $P\left(H_{1}\right)$, are the probabilities of primary user absence and presence respectively. The second case of throughput is less likely to happen since the detection probability has to be high to avoid the harmful interference to the primary user. It is highly likely to have $P_{d}>0.9$ in conservative systems where the priority is given to primary user protection. In addition, the interference generated by the primary signal significantly limits the secondary network throughput.

To determine the probability of the primary user absence $P\left(H_{0}\right)$, the licensed channel usage (primary user activity) needs to be modeled. The ON/OFF model will be considered in our approach. In this model, the primary user activity is modeled as independent 
and identical distributed random processes where the $\mathrm{ON}$ and $\mathrm{OFF}$ states represents the busy and idle periods of the licensed channel $[13,19]$. The duration of the idle and busy periods are assumed to be exponentially distributed with a mean $\tau_{O F F}$ and $\tau_{O N}$ respectively. Therefore, the probability of primary user absence can be defined as:

$$
P\left(H_{0}\right)=\frac{\tau_{O F F}}{\tau_{O F F}+\tau_{O N}}
$$

As a performance measure, we will use the per node throughput capacity to show how the per-node throughput varies with the cluster parameters such as number of clusters, cluster size, and sensing time. The per node throughput represents the average number of bits per second that can be transmitted from the source node to the destination node. In [120], the per node throughput capacity for a cluster-based wireless sensor network, $\mathcal{T}_{n}$, is obtained as $\Theta(M \mathcal{R} / L)$ for a single-hop clustering and $\Theta\left(\mathcal{R} \sqrt{\frac{M^{3}(\log L-\log M)}{L^{3}}}\right)$ for multihop clustering. However, in the $\mathrm{CR}$ networks, the sensing parameters such as sensing efficiency and primary user activity must be considered for throughput calculations. In this work, we extend the per-node throughput given by [120] to include the spectrum sensing parameters that largely effect the network performance. Accordingly, the pernode throughput is defined by:

$$
\mathcal{T}=\eta \mathcal{T}_{n} \frac{\tau_{O F F}}{\tau_{O F F}+\tau_{O N}}\left(1-P_{f}\right)
$$

It can be shown that the per node throughput capacity depends on the sensing effi- 
ciency, the number of clusters, the probability of false alarm, and the total nodes in the network.

\subsection{Performance Evaluation}

We assumed a cognitive radio network of a 100 terminals deployed in an area of $100 \mathrm{~m} \mathrm{x}$ $100 \mathrm{~m}$. The sensing frame, $T$, is assumed to be fixed and assigned a value of $20 \mathrm{~ms}$. The same power is assumed to be adopted by each terminal for intra-cluster communications with the power control capability which is required for the communication between cluster heads and fusion center.

We conducted several trials with random deployment of CR terminals. In each trial, the cluster radius varied and the percentage of the disconnected node is recorded. An average number of disconnected node over these trial is illustrated in Fig. 6.3 for 1, 2, and 3-hop clustering scheme. As it can be shown, a fraction of $\kappa<0.001$ can be achieved with a cluster radius of $15 \mathrm{~m}, 11 \mathrm{~m}$, and $9 \mathrm{~m}$ for 1-hop, 2-hop, and 3-hop clustering scheme respectively. In Fig. 6.4, the percentage of the disconnected nodes is plotted vs the cluster radius with different node densities for a single-hop clustering scheme.

To demonstrate the effect of the cluster size $N c$, on the detection accuracy, the ROC curves are plotted in Fig. 6.5 for a cluster size of 5, 10, and 15 nodes. The reporting channel conditions is realized with probability of error, $P_{e}$ equals to 0.0001 . Clearly, the detection accuracy improves by increasing the number of nodes in each cluster. However, the cluster size must be upper bounded to prevent network bottlenecks resulting from 


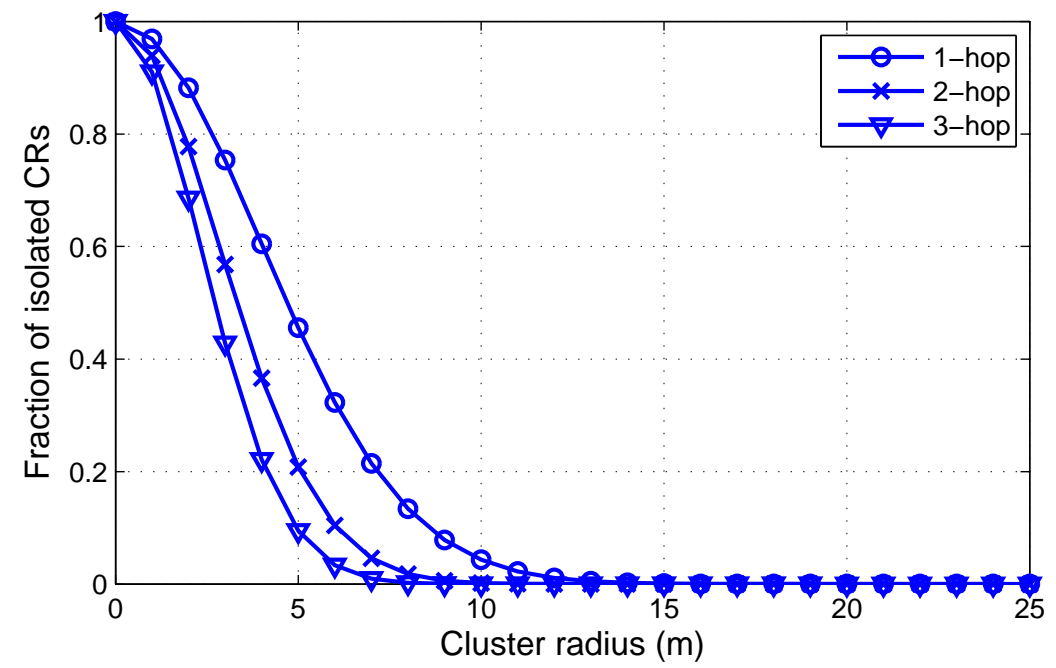

Figure 6.3: fraction of single-node clusters vs cluster radius for 1, 2, 3-hop clusters

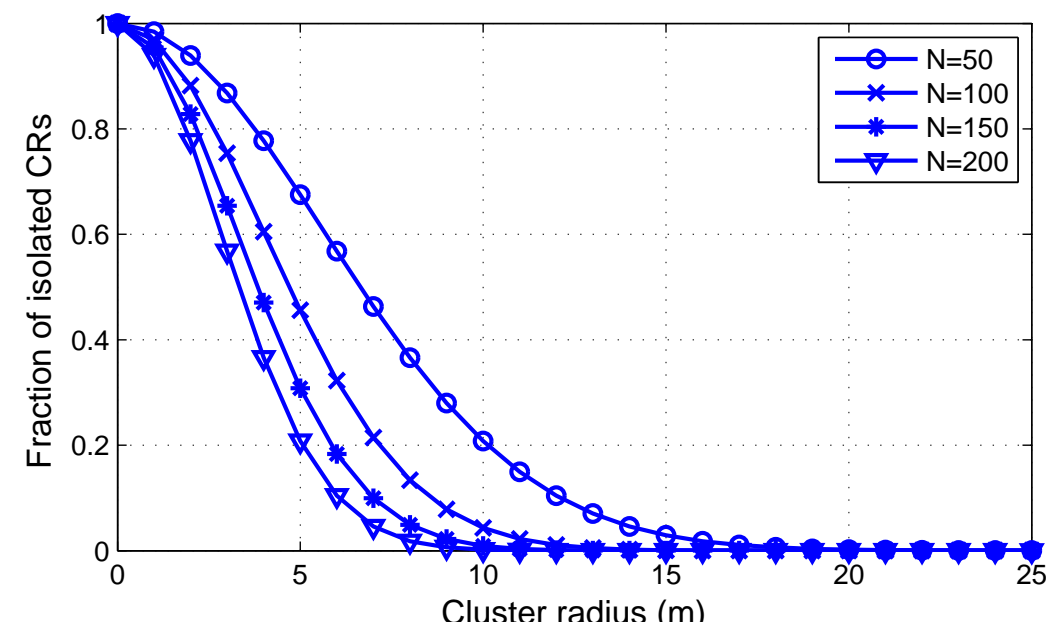

Figure 6.4: Fraction of single-node clusters vs cluster radius under different node densities 
overcrowded clusters.

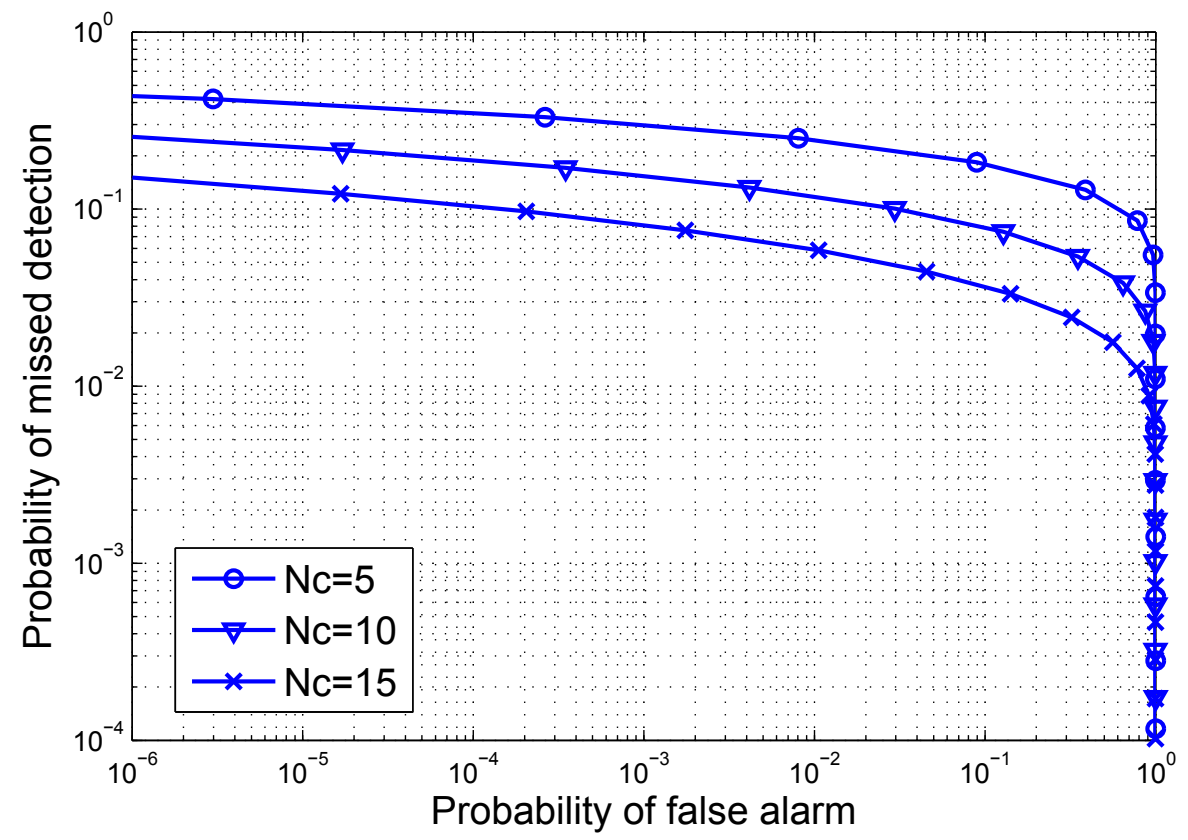

Figure 6.5: Cluster-based cooperative sensing performance with various cluster size, $u=$ $12, \bar{\gamma}_{s}=10 \mathrm{~dB}$

Fig. 6.6 shows that probability of false alarm, $P_{f}$, initially decreases rapidly when the number of cooperative clusters increases. However, it increases later with more cluster heads involved in the cooperative process. In fact, after the initial drop, $P_{f}$ increases with a rate that greatly depends on the probability of error. When the probability of false alarm, $P_{f \mathcal{C}}^{j}$, becomes very small compared to the probability of errors, $P_{e}$, equation (6.14) can be reduced to $P_{f}=1-\prod_{j=1}^{M}\left(1-P_{e}^{j}\right)$ and that explains why $P_{f}$ becomes mainly dependent on $P_{e}$. An optimal number of cluster heads that gives a minimum probability of false alarm is obtained which varies according to the reporting channel probability of error.

To investigate the throughput performance of the proposed system, a target detec- 


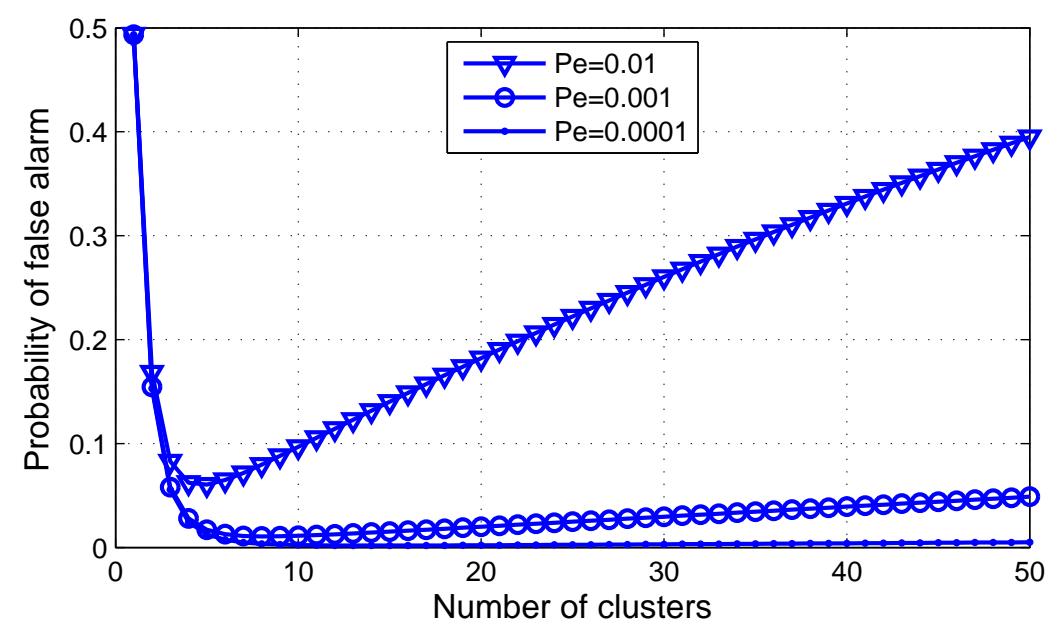

Figure 6.6: False alarm vs cluster size $u=12, \bar{\gamma}_{s}=10 \mathrm{~dB}$

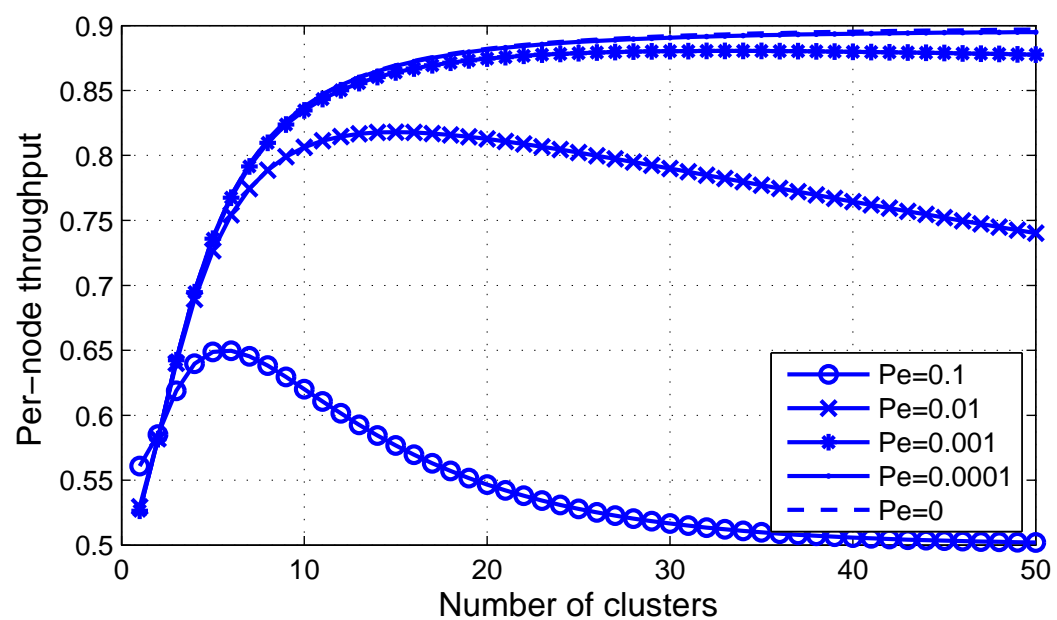

Figure 6.7: The normalized per-node throughput vs number of cooperative clusters, $u=12, \bar{\gamma}_{s}=10 \mathrm{~dB}$

tion probability of 0.9 is used with a mean value of 0.7 and 0.3 for the idle and the active periods respectively. In Fig. 6.7 the normalized per-node throughput is plotted vs the number of clusters for reporting channel probability of error, $P_{e}$ equals $0.1,0.01$, 0.001, and 0.0001. The error-free reporting channel $P_{e}=0$ is also plotted for comparison. The figure reveals that at a higher probability of error the throughput performance 
deteriorates with more users added to the cooperative decision. This can be easily understood from Fig. 6.6 where the probability of false alarm increases more rapidly at higher probability of error.

To compare the cluster-based system with the conventional system, the per-node throughput performance is plotted in Fig. 6.8 for $P_{e}=0.1$. Clearly, the cluster-based system outperforms the non-cluster system especially when more users are engaged in the cooperative process. However, the throughput performance for the cluster-based approach is close to that of the non-cluster approach at the optimal value. This can be explained by the fact the number of cooperative users where the optimal throughput happened is 6 users only. When such small number is deployed in a field, most of the clusters are expected to be single-node clusters and consequently the obtained results come close to that of the non-clustered one. However, we must consider the fact that the number of the deployed secondary users is not necessary be at the optimal value all the times. Therefore, when the number of cooperative users increases, a significant improvement is obtained compared to the non-cluster approach as can be seen from Fig. 6.8. It is worth noting that by adjusting the cluster radius, the number of clusters in the minimal cluster set can be adjusted to match the requirement of both the reporting channel bandwidth and the optimal achievable per-node throughput. Fig. 6.9 shows that a minimal dominating set of 6 clusters is achieved with cluster radius of $20 \mathrm{~m}$ and $12 \mathrm{~m}$ for 1-hop and 2-hop clustering scheme respectively. 


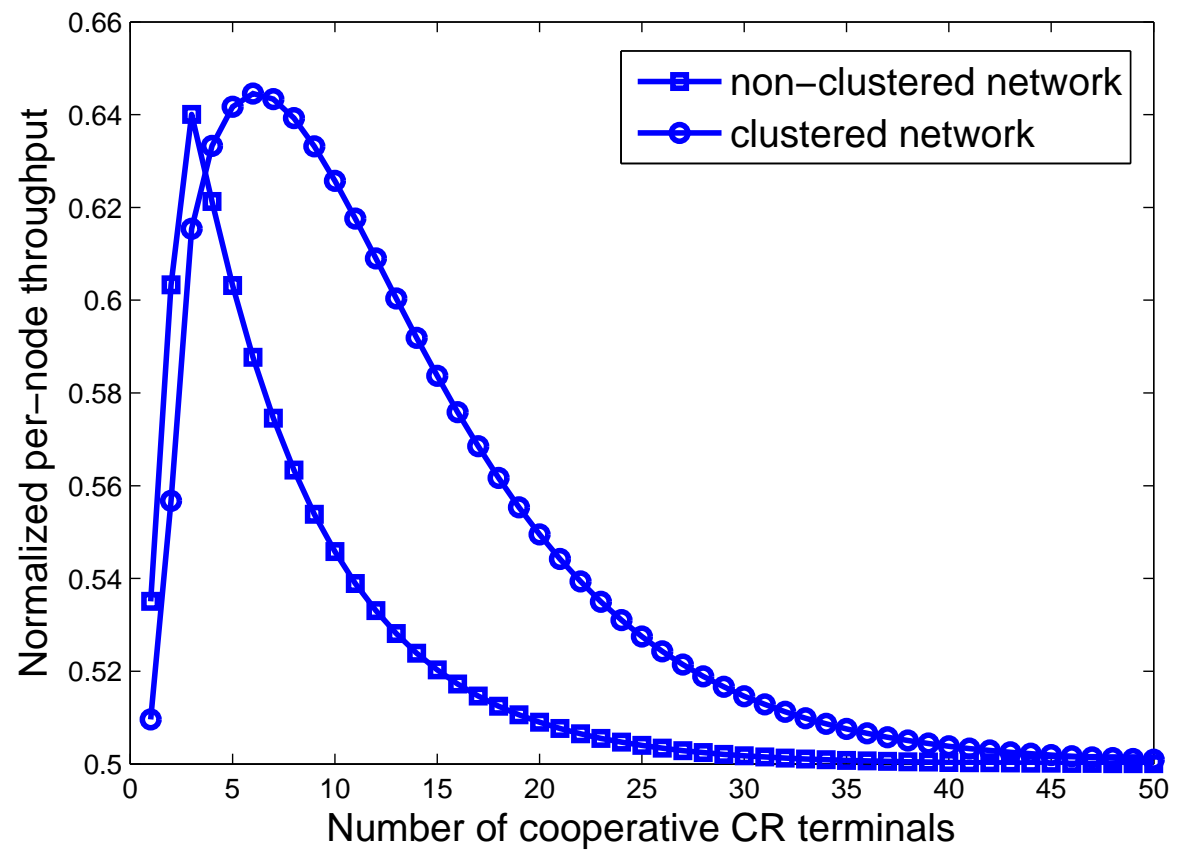

Figure 6.8: Per-node throughput performance of clustered and non-clustered network vs number of cooperative terminals, $u=12, \bar{\gamma}_{s}=10 \mathrm{~dB}$

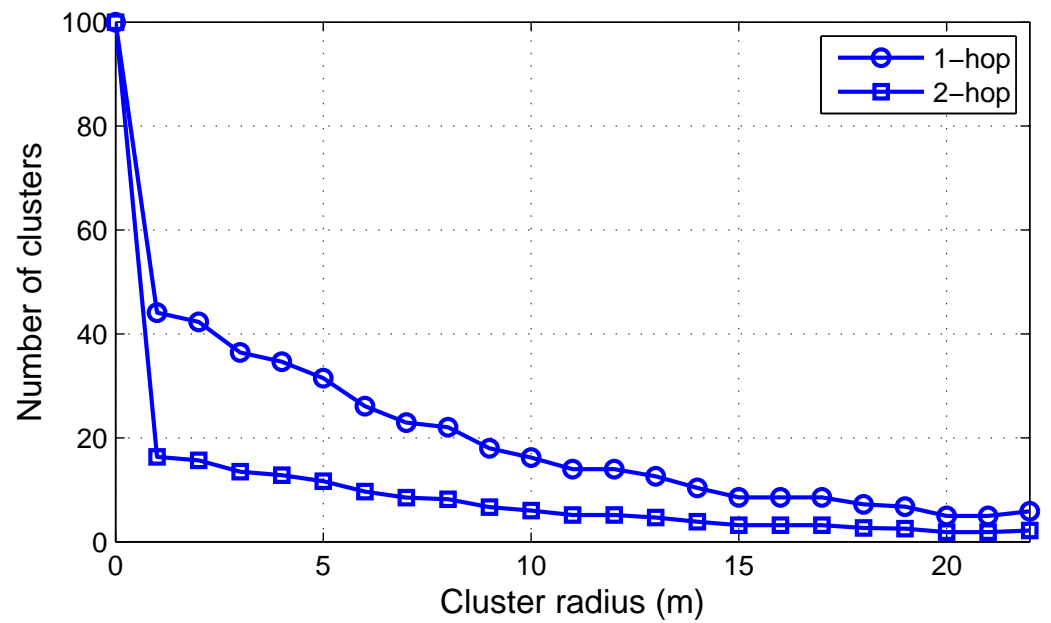

Figure 6.9: The minimal dominating set vs cluster radius for 1 and 2-hop clustering 


\section{Chapter 7}

\section{Conclusions and Future Works}

\subsection{Conclusions}

This dissertation investigates some challenges of CSS over fading channels and proposes new approaches to improve detection accuracy. Our comprehensive evaluations show the importance of including the relaying links and the combining techniques into the performance analysis of CR networks. Closed-form expressions are derived for the average detection probability and the average false alarm probability. The derived expressions can be used to determine the energy threshold value, the minimum number of collected energy samples, and the maximum transmission power that meet a given detection accuracy over non-identical fading channels. The dissertation points out the inconsistency of several assumptions that are typically used for performance analysis of CR networks and show that multipath fading heavily contributes to the unreliability of primary user detection, 
causing fairly large deviations from the primary user detection based on AWGN and path loss models.

The presented results provide a preliminary basis upon which more comprehensive models of cooperative sensing, as needed for spectrum sharing in dynamic spectrum access systems, may be built. Moreover, the derived closed-form expressions for the average false alarm probability and the average detection probability could lead to intuitive system design guidelines. The study also reveals that: i) cooperation allows independently faded radios to collectively achieve robustness to severe fades, ii) multipath fading on relaying channels yields similar performance degradations as multipath fading on sensing channels, iii) a small number of radios are enough to achieve practical detection levels, and iv) practical performance measures for dealing with fading strongly depend on the target probability of detection.

Through extensive analytical and simulation results, we validate the accuracy of the derived closed-form expressions. Moreover, we analyzed the way the detection accuracy varies with the number of diversity branches, the fading severity, and the relay power constraint. In chapter 3, we analyzed the performance of an AF strategy for CSS system with diversity reception and channels impaired with Nakagami- $m$ fading. Compared to the non-cooperative spectrum sensing, a gain of up to $8 \mathrm{~dB}$ is achieved with 4 cooperative users and EGC receiver. Similar set up achieved a gain of $5 \mathrm{~dB}$ with $\mathrm{SC}$ receiver. The most interesting observation is that there is no need to increase the relaying power if a target detection probability is achieved since the improvements gained beyond that are 
not significant.

In chapter 4, we investigate a DAF relaying strategy and compare its performance with an AF relaying strategy. We observed that the DAF strategy outperforms the AF strategy in the all suggested scenarios. The study shows that with DAF strategy, refraining the heavily faded relays improves the detection accuracy while reducing the bandwidth requirement of the relaying links. A gain of $3 \mathrm{~dB}$ is achieved by switching from AF strategy to DAF strategy with 3 cooperative users. The difference in the performance of the DAF and AF strategies becomes more distinguishable as the number of cooperative users increases. Obviously, the system performance, deteriorates if the number of the heavily faded users becomes large enough to induce a missed detection in the final decision.

In chapter 5, we analyze a CSS approach where dual-hop channel statistics, AF relaying, and a selection combining technique are considered. The sensing and the relaying channels are assumed to be subjected to Rayleigh fading. Through extensive analytical and simulation results, the detection accuracy of the proposed CSS is investigated for different levels of the relaying channel SNR and for a different number of cooperative secondary users. Our results show that at a poor reporting channel SNR (less than $5 \mathrm{~dB}$ ), more diversity $(\mathrm{L}=5)$ helps achieve a better detection probability. Moreover, the AoF is reduced by 5 folds when $\bar{\gamma}_{r}=2 \bar{\gamma}_{S}$ compared to the case when $\bar{\gamma}_{r}=0.5 \bar{\gamma}_{s}$, indicating the importance of having the relaying channel power greater than the sensed power. Small AoF means less SNR fluctuations and better detection accuracy. 
In chapter 6 , we investigate a distributed spectrum sensing scheme that eliminates the need for a base station and replace it with a local master fusion center. A lower bound of the cluster radius that keeps the number of isolated nodes under an upper limit is determined in this work. The influence of the cluster size, number of cluster, sensing time, and the probability of reporting channel errors on the per-node throughput capacity is investigated. The results obtained reveal that under bad channel conditions, it is not necessary to include all the cooperative user for the best performance. Instead, an optimal number of clusters that gives a minimum probability of false alarm and consequently a maximum per-node throughput is obtained. When this optimal number matches the minimal dominating set, the reporting channel bandwidth requirements is also achieved.

\subsection{Suggestions for Future Works}

This dissertation addressed some challenges of CSS in CR networks. At this is a very active research area, there are many other challenges and unsolved problems. The nonidentical Nakagami- $m$ fading channels are considered in this dissertation. However, the performance of CSS needs to be investigated over composite fading/shadowing channels. The analysis may include effects of Nakagami-m fading, gamma shadowing, identical, and non-identical. While we mainly discussed the detection accuracy using closed-form expressions for the probability of detection and the probability of false alarm as performance measures, closed-form expressions can also be derived for the outage probability, co-channel interference, and channel capacity to investigate how these metrics are affected 
by the fading/shadowing environment.

It will be important to consider all possible operating conditions such as correlated fading/shadowing channels. For example, the performance of CSS can be analyzed over sensing/relaying channels subjected to correlated Log-Normal shadow-fading in all wireless links of the cooperative network. The independent fading channels are assumed in this dissertation and in the majority of proposed studies in open literature. However, when CR users are deployed in very small areas, the sensing data detected by different users may be highly correlated because neighboring users are likely to be located within almost the same transmission range of the primary transmitter.

The ON/OFF approach is considered in our study to model the activity of the primary user. However, the occupancy history of the spectrum bands by the primary users may vary with time of the day and location. Therefore, it desirable to design algorithms that learn the characteristic of the primary user activity and accordingly alerts its spectrum selection and data transmission strategy. Obviously, the problem of constructing detailed channel occupancy needs further research, so that different times of the day and different locations traversed by the mobile $\mathrm{CR}$ user can be incorporated. The probabilistic spectrum selection algorithm that uses this history may be designed to guarantee performance bounds during long-term operation. 
Appendices 


\section{Appendix A}

\section{A.1 Derivation of $\bar{P}_{f_{i}}$ for a single-relay system}

Substituting $f_{G_{i}}(g)$ from (3.7) into (3.10) yields

$$
\bar{P}_{f_{i}}=\left(\frac{m_{i}}{\bar{g}_{i}}\right)^{m_{i}} \frac{1}{\Gamma\left(m_{i}\right)} \int_{0}^{\infty} \frac{1}{\Gamma\left(m_{i}\right)} \Gamma\left(m_{i}, \frac{m_{i} \lambda}{N_{0}\left(1+A_{i} g\right)}\right) g^{m_{i}-1} e^{-\left(\frac{m_{i}}{\bar{g}_{i}}\right) g} d g
$$

Using the series summation $\Gamma(m, x)=(m-1) ! e^{-x} \sum_{n=0}^{m-1} \frac{x^{n}}{n !}[105$, Eq. 8.352.2], with the fact that $(m-1) !=\Gamma(m),($ A.1) becomes

$$
\bar{P}_{f_{i}}=\left(\frac{m_{i}}{\bar{g}_{i}}\right)^{m_{i}} \frac{1}{\Gamma\left(m_{i}\right)} \sum_{q=0}^{m_{i}-1} \frac{1}{q !}\left(\frac{m_{i} \lambda}{N_{0}}\right)^{q} \int_{0}^{\infty}\left(1+A_{i} g\right)^{-q} g^{m_{i}-1} e^{-\frac{m_{i} \lambda}{N_{0}\left(1+A_{i} g\right)}} e^{-\left(\frac{m_{i}}{\bar{g}_{i}}\right) g} d g
$$

Using $e^{-\frac{a}{b+x}}=\sum_{k=0}^{\infty} \frac{(-1)^{k} a^{k}}{k !(b+x)^{k}}$, we have

$$
\bar{P}_{f_{i}}=\left(\frac{m_{i}}{\bar{g}_{i}}\right)^{m_{i}} \frac{1}{\Gamma\left(m_{i}\right)} \sum_{q=0}^{m_{i}-1} \frac{1}{q !}\left(\frac{m_{i} \lambda}{N_{0}}\right)^{q} \sum_{n=0}^{\infty} \frac{(-1)^{n}}{n !}\left(\frac{m_{i} \lambda}{N_{0}}\right)^{n} \int_{0}^{\infty}\left(1+A_{i} g\right)^{-(q+n)} g^{m_{i}-1} e^{-\left(\frac{m_{i}}{\bar{g}_{i}}\right) g} d g .
$$


The above integral is evaluated with the aid of $\int_{0}^{\infty} e^{-p x} x^{q-1}(1+a x)^{-v} d x=\frac{\Gamma(q)}{a^{q}} U(q ; q+$ $\left.1-v ; \frac{p}{a}\right)[105$, Eq. 3.383 .5$]$ to yield the result shown in (3.11).

\section{A.2 Derivation of $\bar{P}_{f_{S C}}$}

Under non-identical fading, the general form for the $\operatorname{PDF} f_{G_{m a x}}(g)$ is given by $[93,121]$

$$
f_{G_{\max }}(g)=\sum_{j=1}^{L} f_{G_{j}}(g) \underbrace{\prod_{i=1}^{L} F_{G_{i}}}_{i \neq j}(g)
$$

where, $F($.$) denotes the CDF. The above PDF is evaluated for integer values of m$ in [122] as follows

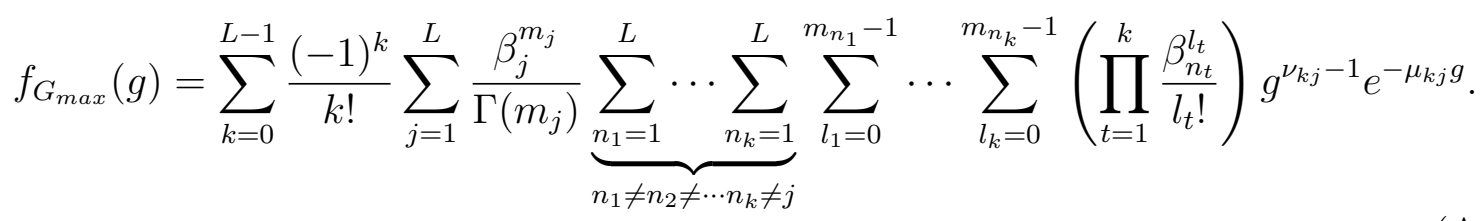

Using (A.5), (3.14) can be expressed as follows

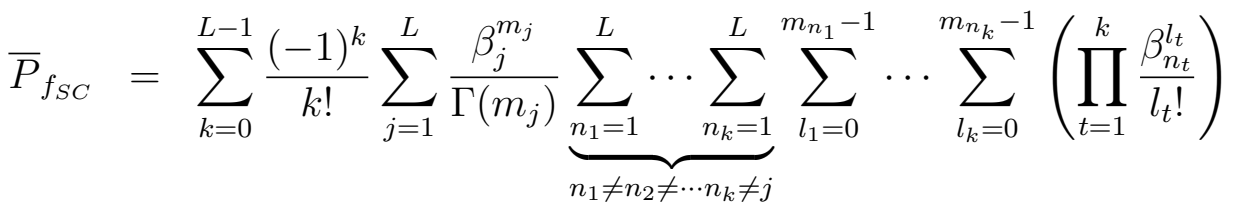

$$
\begin{aligned}
& \int_{0}^{\infty} \frac{1}{\Gamma\left(m_{S C}\right)} \Gamma\left(m_{S C}, \frac{m_{S C} \lambda}{N_{0}\left(1+A_{S C} g\right)}\right) g^{\nu_{k j}-1} e^{-\mu_{k j} g} d g .
\end{aligned}
$$


Using the series summation $\Gamma(m, x)=(m-1) ! e^{-x} \sum_{n=0}^{m-1} \frac{x^{n}}{n !}$ and the fact that $e^{-\frac{a}{b+x}}=$ $\sum_{k=0}^{\infty} \frac{(-1)^{k} a^{k}}{k !(b+x)^{k}}$ in a way similar to Appendix A.1, we have

$$
\begin{aligned}
\bar{P}_{f_{S C}}= & \sum_{k=0}^{L-1} \frac{(-1)^{k}}{k !} \sum_{j=1}^{L} \frac{\beta_{j}^{m_{j}}}{\Gamma\left(m_{j}\right)} \underbrace{}_{n_{n_{1} \neq n_{2} \neq \cdots n_{k} \neq j}^{L} \cdots \sum_{n_{k}=1}^{L} \sum_{l_{1}=0}^{m_{n_{1}}-1} \cdots \sum_{l_{k}=0}^{m_{n_{k}}-1}\left(\prod_{t=1}^{k} \frac{\beta_{n_{t}}^{l_{t}}}{l_{t} !}\right)^{m_{j}-1} \frac{1}{q !}\left(\frac{m_{S C} \lambda}{N_{0}}\right)^{q}} \\
& \sum_{n=0}^{\infty} \frac{(-1)^{n}}{n !}\left(\frac{m_{S C} \lambda}{N_{0}}\right)^{n} \int_{0}^{\infty}\left(1+A_{S C} g\right)^{-(q+n)} g^{\nu_{k j}-1} e^{-\mu_{k j} g} d g .
\end{aligned}
$$

Using $\int_{0}^{\infty} e^{-p x} x^{q-1}(1+a x)^{-v} d x=\frac{\Gamma(q)}{a^{q}} U\left(q ; q+1-v ; \frac{p}{a}\right)[105$, Eq. 3.383.5], to evaluate the integral in (A.7), the desired result is obtained as in (3.15).

\section{A.3 Derivation of $\bar{P}_{f_{E G C}}$}

Since $g_{i}$ 's are independently distributed and each follows the gamma distribution given in (3.7), the moment generating function of $\sum_{i}^{L} g_{i}$, is given by

$$
M_{G}(t)=\prod_{i=1}^{L} M_{G_{i}}(t)=\prod_{i=1}^{L}\left(1-\beta_{i} t\right)^{-m_{i}}
$$

where $M_{G_{i}}(t), i=1,2, \cdots, L$ is the moment generating function of the channel gain $g_{i}$. To derive a PDF for the random variable $R$, we define a new random variable $w_{i}=A_{i} g_{i}$.

Obviously, $\beta_{w_{i}}=\frac{m_{i}}{A_{i} \bar{g}_{i}}=\frac{\beta_{i}}{A_{i}}$ and the moment generating function of $R=\sum_{i}^{L} w_{i}$ can be expressed as

$$
M_{R}(t)=\prod_{i=1}^{L} M_{W_{i}}(t)=\prod_{i=1}^{L}\left(1-\frac{\beta_{i}}{A_{i}} t\right)^{-m_{i}}
$$


Using a general partial fraction technique to compute the inverse of $M_{R}(t)$ and following the same steps as in [101], the PDF of $R$ for integer values of $m_{i}$ is obtained as follows

$$
f_{R}(r)=\left[\prod_{j=1}^{L}\left(-\frac{\beta_{i}}{A_{i}}\right)^{m_{j}}\right] \sum_{j=1}^{L} \sum_{v=1}^{m_{j}} \frac{(-1)^{v} b_{j v} r^{v-1} e^{-\frac{\beta_{j}}{A_{j}} r}}{(v-1) !}
$$

The PDF given in (A.10) requires all the $\beta_{i}$ 's to be distinct and not necessary to be integers. If some of the $\beta_{i}$ 's are equal then the corresponding factors can be combined. Substituting (A.10) into (3.26) yields

$$
\bar{P}_{f_{E G C}}=\left[\prod_{j=1}^{L}\left(-\frac{\beta_{i}}{A_{i}}\right)^{m_{j}}\right] \sum_{j=1}^{L} \sum_{v=1}^{m_{j}} \frac{(-1)^{v} b_{j v}}{(v-1) !} \int_{0}^{\infty} \frac{1}{\Gamma\left(m_{j}\right)} \Gamma\left(m_{j}, \frac{m_{j} \lambda}{N_{0}(1+r)}\right) r^{v-1} e^{-\frac{\beta_{j}}{A_{j}} r} d r .
$$

Following the same steps in Appendices A.1 and A.2, to evaluate the above integral, (A.11) becomes

$$
\bar{P}_{f_{E G C}}=\left[\prod_{j=1}^{L}\left(-\frac{\beta_{i}}{A_{i}}\right)^{m_{j}}\right] \sum_{j=1}^{L} \sum_{v=1}^{m_{j}}(-1)^{v} b_{j v} \sum_{q=0}^{m_{j}-1} \frac{1}{q !}\left(\frac{m_{i} \lambda}{N_{0}}\right)^{q} \sum_{n=0}^{\infty} \frac{(-1)^{n}}{n !}\left(\frac{m_{i} \lambda}{N_{0}}\right)^{n} U\left(v ; v+1-q-n ; \frac{\beta_{j}}{A_{j}}\right) .
$$




\section{Appendix B}

\section{B.1 Derivation of $\bar{P}_{f}^{D A F}$}

Using the series summation $\Gamma(m, x)=(m-1) ! e^{-x} \sum_{n=0}^{m-1} \frac{x^{n}}{n !}[105$, Eq. 8.352.2], with the fact that $(m-1) !=\Gamma(m),(4.20)$ becomes

$$
\begin{aligned}
\bar{P}_{f}^{D A F}= & {\left[\prod_{l=1}^{L}\left(-\frac{\beta_{l}}{A_{l}}\right)^{m_{l}}\right]\left[\prod_{l=1, \hat{\theta}_{l}^{j} \neq 0}^{L}\left(-\frac{\beta_{l}}{A_{l}}\right)^{m_{l}}\right] \sum_{i=1, \hat{\theta}_{i}^{j} \neq 0}^{L} \sum_{v=1}^{m_{i}} \frac{(-1)^{v} b_{i v}}{(v-1) !} \sum_{q=0}^{m_{i}-1} \frac{1}{q !}\left(\frac{m_{i} \lambda}{N_{0}}\right)^{q} \int_{0}^{\text {infty }}(1+r)^{-q} } \\
& e^{-\frac{m_{i} \lambda}{N_{0}(1+r)}} r^{v-1} e^{-\frac{\beta_{i}}{A_{i}} r} d r .
\end{aligned}
$$

Using $e^{-\frac{a}{b+x}}=\sum_{k=0}^{\infty} \frac{(-1)^{k} a^{k}}{k !(b+x)^{k}}$, we have

$$
\begin{aligned}
\bar{P}_{f}^{D A F}= & {\left[\prod_{l=1}^{L}\left(-\frac{\beta_{l}}{A_{l}}\right)^{m_{l}}\right]\left[\prod_{l=1, \hat{\theta}_{l}^{j} \neq 0}^{L}\left(-\frac{\beta_{l}}{A_{l}}\right)^{m_{l}}\right] \sum_{i=1, \hat{\theta}_{i}^{j} \neq 0}^{L} \sum_{v=1}^{m_{i}} \frac{(-1)^{v} b_{i v}}{(v-1) !} \sum_{q=0}^{m_{i}-1} \frac{1}{q !}\left(\frac{m_{i} \lambda}{N_{0}}\right)^{q} \sum_{n=0}^{\infty} \frac{(-1)^{n}}{n !} } \\
& \left(\frac{m_{i} \lambda}{N_{0}}\right)^{n} \int_{0}^{\infty}(1+r)^{-(q+n)} r^{v-1} e^{-\left(\frac{\beta_{i}}{A_{i}}\right) r} d r .
\end{aligned}
$$


The above integral is evaluated with the aid of $\int_{0}^{\infty} e^{-p x} x^{q-1}(1+a x)^{-v} d x=\frac{\Gamma(q)}{a^{q}} U(q ; q+$ $\left.1-v ; \frac{p}{a}\right)[105$, Eq. 3.383.5] to yield

$$
\begin{aligned}
\bar{P}_{f}^{D A F}= & \sum_{j=1}^{2^{L}}\left[\prod_{i=1}^{L} \mathbb{P}_{i \mid H_{0}}^{j}\right]\left[\prod_{l=1, \hat{\theta}_{l}^{j} \neq 0}^{L}\left(-\frac{\beta_{l}}{A_{l}}\right)^{m_{l}}\right] \sum_{i=1, \hat{\theta}_{i}^{j} \neq 0}^{L} \sum_{v=1}^{m_{i}}(-1)^{v} b_{i v} \sum_{q=0}^{m_{i}-1} \frac{1}{q !}\left(\frac{m_{i} \lambda}{N_{0}}\right)^{q} \\
& \sum_{n=0}^{\infty}(-1)^{n} b_{n} U\left(v ; v+1-q-n ; \frac{\beta_{i}}{A_{i}}\right)
\end{aligned}
$$

\section{B.2 Derivation of the approximated $\bar{P}_{f}^{D A F}$}

Using the approximated series summation $\Gamma\left(m_{i}, \frac{m_{i}}{N_{0}(1+r)}\right)=\left(m_{i}-1\right) ! e^{\frac{m_{i}}{N_{0} r}},(\mathrm{~A} .11)$ can be rewritten as

$$
\left[\prod_{l=1}^{L}\left(-\frac{\beta_{l}}{A_{l}}\right)^{m_{l}}\right]\left[\prod_{l=1, \hat{\theta}_{l}^{j} \neq 0}^{L}\left(-\frac{\beta_{l}}{A_{l}}\right)^{m_{l}}\right] \sum_{i=1, \hat{\theta}_{i}^{j} \neq 0}^{L} \sum_{v=1}^{m_{i}} \frac{(-1)^{v} b_{i v}}{(v-1) !} \sum_{q=0}^{m_{i}-1} \frac{1}{q !}\left(\frac{m_{i} \lambda}{N_{0}}\right)^{q} \int_{0}^{\infty} r^{v-q-1} e^{-\frac{m_{i} \lambda}{N_{0} r}-\frac{\beta_{i}}{A_{i}} r} .
$$

Using $\int_{0}^{\infty} x^{v-1} e^{-\frac{\beta}{x}-\gamma x} d x=2\left(\frac{\beta}{\gamma}\right)^{\frac{v}{2}} K_{v}(2 \sqrt{\beta \gamma})$ [105, Eq. 3.471.9], to evaluate the integral in (B.4) with some algebra, yields the result shown in (4.25). 


\section{Appendix C}

\section{C.1 Derivation of $f_{\Gamma_{\max }}(\gamma)$}

With the help of the asymptotic form of the first order modified Bessel function of the second order as defined in [123, Eq. 11.124], equation (5.10) can be written as,

$$
\begin{aligned}
f_{X_{\text {max }}}(x)= & \frac{L \beta_{s} \beta_{r}}{2}\left[\left[\sum_{k=0}^{L-1}\left(\begin{array}{c}
L-1 \\
k
\end{array}\right)(-1)^{k} x^{k}\left(\sqrt{\beta_{s} \beta_{r}}\right)^{k} e^{-\frac{k x}{2\left(\beta_{s}+\beta_{r}\right)}}\left(\frac{1}{x \sqrt{\beta_{s} \beta_{r}}}\right)^{k}\right] x e^{-\frac{x}{2\left(\beta_{s}+\beta_{r}\right)}}\right. \\
& {\left.\left[\frac{\beta_{s}+\beta_{r}}{\sqrt{\beta_{s} \beta_{r}}}\left(\frac{1}{x \sqrt{\beta_{s} \beta_{r}}}\right)+2 K_{0}\left(x \sqrt{\beta_{s} \beta_{r}}\right)\right]\right] . }
\end{aligned}
$$

With some simple algebra, (C.1) is evaluated as follows,

$$
f_{X_{\max }}(x)=\frac{L \beta_{s} \beta_{r}}{2} \sum_{k=0}^{L-1}\left(\begin{array}{c}
L-1 \\
k
\end{array}\right)(-1)^{k} e^{-\frac{(k+1) x}{2\left(\beta_{s}+\beta_{r}\right)}}\left[\frac{\beta_{s}+\beta_{r}}{\beta_{s} \beta_{r}}+2 x K_{0}\left(x \sqrt{\beta_{s} \beta_{r}}\right)\right]
$$

Since, the harmonic mean is defined as $X=m_{H}\left(X_{1}, X_{2}\right)=\frac{2 X_{1} X_{2}}{X_{1}+X_{2}}$, the end-to-end SNR of the dual-hop system can be written as $\gamma_{e}=m_{H}\left(X_{1}, X_{2}\right) / 2$. Therefore, the following 
transformation of variable is required,

$$
f_{\Gamma_{\max }}(\gamma)=2 f_{X_{\max }}(2 \gamma)
$$

If we set $\beta_{s}=1 / \bar{\gamma}_{s}$ and $\beta_{r}=1 / \bar{\gamma}_{r}$ and apply the transformation given in (C.3) to (C.2) , the desired result is obtained as in (5.11).

\section{C.2 Derivation of $\bar{P}_{d}$ over i.i.d Rayleigh channels}

Substituting equation (5.11) of theorem 1 in equation (5.5) yields,

$$
\begin{aligned}
\bar{P}_{d}= & \frac{L}{\bar{\gamma}_{s} \bar{\gamma}_{r}} \sum_{k=0}^{L-1}\left(\begin{array}{c}
L-1 \\
k
\end{array}\right)(-1)^{k}\left[\left(\bar{\gamma}_{s}+\bar{\gamma}_{r}\right) \int_{0}^{\infty} e^{-\frac{(k+1) \bar{\gamma}_{s} \bar{\gamma}_{r}}{\bar{\gamma}_{s}+\bar{\gamma}_{r}} \gamma} Q_{u}(\sqrt{a \gamma}, \sqrt{\lambda}) \mathrm{d} \gamma\right. \\
& \left.+4 \int_{0}^{\infty} \gamma e^{-\frac{(k+1) \bar{\gamma}_{s} \bar{\gamma}_{r}}{\bar{\gamma}_{s}+\bar{\gamma}_{r}}} K_{0}\left(\gamma \frac{2}{\sqrt{\bar{\gamma}_{s} \bar{\gamma}_{r}}}\right) Q_{u}(\sqrt{a \gamma}, \sqrt{\lambda}) \mathrm{d} \gamma\right] .
\end{aligned}
$$

The first integral in (C.4) is evaluated with help of [124, Eq. 12] and change of variable $y=\sqrt{\gamma}$ which yields,

$$
\begin{aligned}
\int_{0}^{\infty} 2 y e^{-\frac{(k+1) \bar{\gamma}_{s} \bar{\gamma}_{r}}{\bar{\gamma}_{s}+\bar{\gamma}_{r}} y^{2}} Q_{u}(\sqrt{a} y, \sqrt{\lambda}) \mathrm{dy}= & \frac{2}{p} e^{-\frac{\lambda}{2}}\left[\left(\frac{p+a}{a}\right)^{u-1}\left(e^{\frac{\lambda a}{2(p+a)}}-\sum_{n=0}^{u-2} \frac{1}{n !}\left(\frac{\lambda a}{2(p+a)}\right)^{n}\right)\right. \\
& \left.+\sum_{n=0}^{u-2} \frac{1}{n !}\left(\frac{\lambda}{2}\right)^{n}\right]
\end{aligned}
$$


where, $p=2 \frac{(k+1) \bar{\gamma}_{s} \bar{\gamma}_{r}}{\bar{\gamma}_{s}+\bar{\gamma}_{r}}$. For the second integration, we employed the same technique used in [49] to approximate the generalized Marcum-Q function as follows:

$$
Q_{u / 2}(\sqrt{a \gamma}, \sqrt{\lambda}) \approx \frac{e^{-\frac{a}{2} \gamma}}{\gamma^{\frac{u-1}{2}}} \sum_{i=1}^{N} \Psi_{i} I_{u-1}\left(\sqrt{a \gamma}\left(t_{i}+\sqrt{\lambda}\right)\right)
$$

where, $\Psi_{i}=\frac{w_{i} e^{t_{i}}\left(t_{i}+\sqrt{\lambda}\right)^{u} e^{-\frac{\left(t_{i}+\sqrt{\lambda}\right)^{2}}{2}}}{a^{\frac{u-1}{2}}}$, and $w_{i}$ and $t_{i}$ are weight factors and abscissas $(i=$ $1, \ldots, N)$ of the Gaussian Laguerre integration, respectively [106, Table 25.9]. With this approximation, the second integration becomes:

$$
\sum_{i=1}^{N} \Psi_{i}\left(\left(\frac{\sqrt{a}\left(t_{i}+\sqrt{\lambda}\right)}{2}\right)^{u-1}\right) \int_{0}^{\infty} \gamma^{\frac{-u+3}{2}} e^{-\frac{2(k+1) \bar{\gamma}_{s} \bar{\gamma}_{r}+a\left(\bar{\gamma}_{s}+\bar{\gamma}_{r}\right)}{2\left(\bar{\gamma}_{s}+\bar{\gamma}_{r}\right)}} K_{0}\left(\gamma \frac{2}{\sqrt{\bar{\gamma}_{s} \bar{\gamma}_{r}}}\right) I_{u-1}\left(\sqrt{a \gamma}\left(t_{i}+\sqrt{\lambda}\right)\right) \mathrm{d} \gamma
$$

With the help of the asymptotic form of the modified bessel function given in [123, Eq.

$11.124], I_{v}(x) \approx \frac{1}{\Gamma(v+1)}\left(\frac{x}{2}\right)^{v}$, the second integration can be expressed as,

$$
\int_{0}^{\infty}(.) \mathrm{d} \gamma=\frac{1}{\Gamma(u)} \sum_{i=1}^{N} \Psi_{i}\left(\left(\frac{\sqrt{a}\left(t_{i}+\sqrt{\lambda}\right)}{2}\right)^{u-1}\right) \int_{0}^{\infty} \gamma e^{-\frac{2(k+1) \bar{\gamma}_{s} \bar{\gamma}_{r}+a\left(\bar{\gamma}_{s}+\bar{\gamma}_{r}\right)}{2\left(\bar{\gamma}_{s}+\bar{\gamma}_{r}\right)} \gamma} K_{0}\left(\gamma \frac{2}{\sqrt{\bar{\gamma}_{s} \bar{\gamma}_{r}}}\right) \mathrm{d} \gamma
$$

The above integral can be evaluated with the help of [105, Eq. 6.624.1] to yield,

$$
\int_{0}^{\infty}(.) \mathrm{d} \gamma=\frac{1}{\Gamma(u)}\left(\frac{\sqrt{a}}{2}\right)^{u-1} \sum_{i=1}^{N} \frac{\Psi_{i}\left(t_{i}+\sqrt{\lambda}\right)^{u-1}}{c^{2}-d^{2}}\left[\frac{c}{\sqrt{c^{2}-d^{2}}} \ln \left(\frac{c}{d}+\sqrt{\left(\frac{c}{d}\right)^{2}-1}\right)-1\right]
$$


where, $c=\frac{2(k+1) \bar{\gamma}_{s} \bar{\gamma}_{r}+a\left(\bar{\gamma}_{s}+\bar{\gamma}_{r}\right)}{2\left(\bar{\gamma}_{s}+\bar{\gamma}_{r}\right)}$ and $d=\frac{2}{\sqrt{\bar{\gamma}_{s} \bar{\gamma}_{r}}}$. Substituting (C.5) and (C.9) in (C.4), $\bar{P}_{d}$ can be expressed as shown in (5.12). 


\section{References}

[1] J. Mitola, "Cognitive Radio for Flexible Mobile Multimedia Communications," Mobile Networks and Applications, vol. 6, 2001.

[2] T. Yucek and H. Arslan, "A Survey of Spectrum Sensing algorithms for Cognitive Radio applications," IEEE communications Surveys and tutorials, vol. 11, pp. 116130, First Quarter 2009.

[3] D. Cabric, S. M. Mishra, and R. W. Broderson, "Implementation Issues in Spectrum Sensing for Cognitive Radios," in Conference Record - Asilomar Conference on Signals, Systems and Computers, vol. 1, pp. 772-776, Nov 2004.

[4] I. F. Akyildiz, W.-Y. Lee, M. C. Vuran, and S. Mohanty, "NeXt generation/dynamic spectrum access/cognitive radio wireless networks: a survey," Pervasive and Mobile Computing, vol. 50, pp. 2127-2159, Sept. 2006.

[5] S. Haykin, "Cognitive Radio: Brain-empowered Wireless Communications," Mobile Networks and Applications, vol. 23, pp. 201 - 220, Feb. 2005.

[6] I. F. Akyildiz, W.-Y. Lee, and K. R. Chowdhury, "CRAHNs: Cognitive Radio ad hoc Networks," Ad Hoc Networks, vol. 7, pp. 810-836, July 2009.

[7] M. Gandetto and C. Regazzoni, "Spectrum Sensing: A Distributed Approach for Cognitive Terminals," IEEE Jornal on Selected Areas in Communications, vol. 25, pp. 546-557, April 2007. 
[8] S. Hussain and X. Fernando, "On the Throughput Performance of Cluster-based Cognitive Radio Networks ," International Journal for Computer and Distributed Systems, vol. 8, pp. 307-331, Jan. 2012.

[9] Q. Zhao, L. Tong, and A. Swami, "Decentralized cognitive MAC for Dynamic Spectrum Access," in DySPAN 2005, First IEEE International Symposium onNew Frontiers in Dynamic Spectrum Access Networks, pp. 224-232, Nov 2005.

[10] M. K. Simon and M.-S. Alouini, Digital communication over Fading Channels . John Wiley \& Sons, 2nd Edition, New York, USA, 2005.

[11] A. Ghasemi and E. S. Sousa, "Interference Aggregation in spectrum-Sensing Cognitive Wireless Networks," IEEE Jornal of Selected Topics in Signal Processing, vol. 2, pp. 41-56, Feb 2008.

[12] S. Shankar, "Spectrum Agile Radios: Utilization and Sensing Architecture," in Proc. IEEE 1st Symposium Dynamic Spectrum Access Networks, DySPAN'05, pp. 160-169, Nov. 2005.

[13] W.-Y. Lee and I. F. Akyildiz, "Optimal Spectrum Sensing Framework for Cognitive Radio Networks," IEEE Trans. on Wireless Communications, vol. 7, pp. 3845-3857, Oct. 2008.

[14] C. Chou, S. Shanker, H. Kim, and K. G. Shin, "What and How Much to Gain by Spectrum Agility," IEEE Journal on Selected Area in Communications, vol. 25, no. 3, pp. 576-588, 2007.

[15] H. Kim and K. G. Shin, "Efficient Discovery of Spectrum Opportunuities with MAC-layer Sensing in Cognitive Radio Networks ," IEEE Trans. on Mobile Computing, vol. 7, no. 5, pp. 533-545, 2008.

[16] K. Siriram and W. Whitt, "Characterizing Supperposition Arrival Processes in 
Packet Multiplexers for Voice and Data," IEEE Journal on Selected Area in Communications, vol. 4, no. 6, pp. 833-846, 1986.

[17] H. Celebi, I. Guvenc, S. Gezici, and H. Arslan, "Cognitive Radio Systems for Spectrum, Location, and Environment Awareness," IEEE Antennas and Propagation Magazine, vol. 52, p. 4161, Aug. 2010.

[18] S. Hussain and X. Fernando, "Spectrum sensing in cognitive radio networks: Upto-date techniques and future challenges," in Proc. IEEE Int. Conf. on Scuence and Technology for Humanity, TIC-STH'09, pp. 736-742, Sept. 2009.

[19] A. Ghasemi and E. S. Sousa, "Optimization of Spectrum Sensing for Opportunistic Spectrum Access in Cognitive Radio Networks," in Proc. IEEE Consumer Communications and Networking Conference, pp. 1022 - 1026, Las Vegas, NV, USA, Jan 2007.

[20] S. Hussain and X. Fernando, "Closed-Form Analysis of Relay-Based Cognitive Radio Networks over Nakagami-m Fading Channels," Accepted for publication in IEEE Trans. on Vehicular Technology, July 2013.

[21] S. Hussain and X. Fernando, "Performance Analysis of Relay-Based Cooperative Spectrum Sensing in Cognitive Radio Networks over Non-Identical Nakagami-m Channels," 2nd revision submitted for publication in IEEE Trans. on Communications, May 2013.

[22] C. Xing, B. Zhi-Song, and W. Wei-Ling, "Detection Efficiency of Cooperative Spectrum Sensing in Cognitive Radio Network," in ELESVIER, Journal of China Universities of Posts and Telecommunications, vol. 15, pp. 1-7, 2008.

[23] A. Ghasemi and E. S. Sousa, "Collaborative Spectrum Sensing for Opportunistic Access in Fading Environments," in Proc. IEEE 1st Symposium Dynamic Spectrum Access Networks (DySPAN'05), pp. 131-136, Baltimore, MD, Nov. 2005. 
[24] Z. Quan, S. Cui, and A. H. Sayed, "Optimal Linear Cooperation for Spectrum Sensing in Cognitive Radio Networks," IEEE Jornal of Selected Topics in Signal processing, vol. 2, pp. 28-40, Feb. 2008.

[25] D. Cabric, S. Mishra, and R. Broderson, "Implementation Issues in Spectrum Sensing for Cognitive Radios," in Proc. 38th Asilomar Conf. Signal, Systems, and Computers, pp. 772-776, Pacific Grove, CA, Nov. 2004.

[26] G. Ganesan and Y. G. Li, "Cooperative Spectrum Sensing in Cognitive Radio, Part I: Two User Networks," IEEE Trans. on Wireless Communications, vol. 6, pp. 2204-2213, June 2007.

[27] G. Ganesan and Y. G. Li, "Cooperative Spectrum Sensing in Cognitive Radio, Part II: MultiUser Networks," IEEE Trans. on Wireless Communications, vol. 6, pp. 2214-2222, June 2007.

[28] A. S. Zahmati, S. Hussain, X. N. Fernando, , and A. Grami, "Cognitive Radio Wireless Sensor Networks: Emerging Topics and Recent Challenges," in Proc. IEEE TIC-STH, p. 593596, Toronto, ON, Canada, Sep. 2009.

[29] K. B. Letaief and W. Zhang, "Cooperative Communications for Cognitive Radio Networks," Proceedings of the IEEE, vol. 97, pp. 878-893, May 2009.

[30] F. F. Digham, M.-S. Alouini, and M. K. Simon, "On the Energy Detection of Unknown Signals Over Fading Channels," IEEE Trans. on Communications, vol. 55, pp. 3845-3857, Jan. 2007.

[31] L. Chen, J. Wang, and S. Li, "Cooperative Spectrum Sensing with Multi-Bits Local Sensing Decisions in Cognitive Radio Networks," in IEEE WCNC, pp. 570-575, New Orleans, LA, USA, April 2008.

[32] J. Ma, G. Zhao, and Y. Li, "Soft Combination and Detection for Cooperative 
Spectrum Sensing in Cognitive Radio Networks," IEEE Trans. on Wireless Communications, vol. 7, pp. 4502-4507, Nov. 2008.

[33] M. D. Renzo, L. Imbriglio, F. Grazios, and F. Santucci, "Cooperative Spectrum Sensing over Correlated Log-Normal Sensing and Reporting Channels," in Proc. IEEE GLOBECOM, Honolulu, HI, USA, Dec. 2009.

[34] Q. Chen and M. Motani, "Cooperative Spectrum Senseing Strategies for Cognitive Radio Mesh Networks," IEEE Trans. on Selected Topics in Signal Processing, vol. 5, pp. 56-67, Feb. 2011.

[35] M. D. Renzo, L. Imbriglio, F. Grazios, and F. Santucci, "Distributed Data Fusion over Correlated LogNormal Sensing and Reporting Channels: Application to Cognitive Radio Networks," IEEE Trans. on Wireless Communications, vol. 8, pp. 5813-5821, Dec. 2009.

[36] O. Younis and S. Fahmy, "Distributed Clustering in Ad-hoc Sensor Networks: A Hybrid, energy-efficient Approach," in INFOCOM 2004, vol. 1, pp. 629-640, Hong Kong, China, March 2004.

[37] M. Qin and R. Zimmermann, "An Energy-Efficient Voting-Based Clustering Algorithm for Sensor Networks," in SNPD/SAWN'05, pp. $444-451,2005$.

[38] S. Hussain, A. S. Zahmati, and X. Fernando, "LASA: Low-Energy Adapive Slot Allocation Scheduling Algorithm for Wireless Sensor Networks," in Proc. Sarnoff Symposium, 2009, SARNOFF'09, Princeton, NJ, USA, April 2009.

[39] H. Gong and M. Liu, "A Two Level TDMA Scheduling Protocol with Intra-cluster Coverage for Large Scale Wireless Sensor Network," in International Jornal of computer Science and Network Security, vol. 6, pp. 77-84, 2006.

[40] G. Y. Lazarous, J. Li, and J. Picone, "A Clustere-based Power-efficient MAC 
Scheme for Event-driven Sensing Applications," in ELSEVIER Ad Hoc Networks, vol. 5, pp. 1017-1030, 2007.

[41] E. Perevalov, R. S. Blum, and D. Safi, "Capacity of Clustered Ad Hoc Networks: How large is "Large"," IEEE Trans. on Communications, vol. 54, pp. 1672-1681, Sept 2006.

[42] S. Basagni, "Distributed clustering for ad hoc networks," in Proc. of the International Symposium on Parallel Architectures, Algorithms and Networks, pp. 310-315, Perth/Fremantle, Aust, June 1999.

[43] A. Amis, R. Prakash, T. Vuong, and D. T. Huynh, "MaxMin D-Cluster Formation in Wireless Ad Hoc Networks," in Proc. IEEE International Conference on Computer Communications, vol. 1, pp. 32-41, Tel Aviv, Israel, March 2000.

[44] T. Chen, H. Zhang, G. Maggioand, and I. Chlamtac, "Topology Management in CogMesh: A Cluster-based Cognitive Radio Mesh Network," in Proc. IEEE International Conference on Communications, pp. 6516-6521, Glasgow, UK, June 2008.

[45] S. Hussain and X. Fernando, "A Novel Approach for Cluster-Based Spectrum Sensing over Band-Limited Reporting Channels ," IET Communications, vol. 6, p. 14661474 , July 2012.

[46] E. Peh and Y.-C. Liang, "Optimization for Cooperative Sensing in Cognitive Radio Networks," in IEEE Wireless Communications and Networking Conference, WCNC'07, pp. 27-32, Kowloon, China, March 2007.

[47] Q. Chen, F. Gao, A. Nallanathan, and Y. Xin, "Improved Cooperative Spectrum Sensing in Cognitive Radio," IEEE Vehicular Technology Conference, vol. 2, pp. 1418-1422, May 2008. 
[48] Y.-C. Liang, Y. Zeng, E. C. Y. Peh, and A. T. Hoang, "Sensing-Throughput Tradeoff for Cognitive Radio Networks," IEEE Trans. on Wireless Communications, vol. 7, pp. 1325-1337, April 2008.

[49] S. Atapattu, C. Tellambura, and H. Jiang, "Performance of an Energy Detector over Channels with Both Multipath Fading and Shadowing," IEEE Trans. on Wireless Communications, vol. 9, pp. 3662-3670, Dec. 2010.

[50] S. P. Hearth, N. Rajatheva, and C. Tellambura, "Energy Detection of Unknown Signals in Fading and Diversity Reception," IEEE Trans. on Communications, vol. 59, pp. 2443-2453, Sep. 2011.

[51] W. Zhang and K. B. Letaief, "Coperative Spectrum Sensing with Transmit and Relay Diversity in Cognitive Radio Networks," IEEE Trans. on Wireless Communications, vol. 7, pp. 4761-4766, Dec. 2008.

[52] J. Lee, Y. Kim, S. Sohn, and J. Kim, "Weighted-Cooperative Spectrum Sensing Scheme using Clustering in Cognitive Radio Systems," in Proc. International Conference on Advanced Communication Technology, ICACT'08, vol. 1, pp. 786-790, Gangwon-Do, South Korea, Feb 2008.

[53] C. Sun, W. Zhang, and K. B. Letaief, "Cluster-Based Cooperative Spectrum Sensing in Cognitive Radio Systems," in Proc. IEEE International Conference on Communications, ICC'07, pp. 2511 - 2515, Glasgow, UK, June 2007.

[54] "Wolfram, the wolfram functions site. [online]. available: http://functions.wolfram.com," 2010.

[55] A. Sandonaris, E. Erkip, and B. Aazhang, "User Cooperation Diversity-Part I: System description," IEEE Trans. on Communications, vol. 51, pp. 1927-1938, Nov. 2003. 
[56] A. Sandonaris, E. Erkip, and B. Aazhang, "User Cooperation Diversity-Part II: Implementation Aspects and Performance Analysis," IEEE Trans. on Communications, vol. 51, pp. 1939-1948, Nov. 2003.

[57] S. M. Mishra, A. Sahai, and R. W. Brodersen, "Cooperative Sensing among Cognitive Radios," in Proc. IEEE Intl. Conference on Communications, ICC'06, vol. 4, pp. 1658-1663, Istanbul, Turkey 2006.

[58] G. Ganesan and Y. Li, "Cooperative Spectrum Sensing in Cognitive Radio, Part I: Two User networks," IEEE Transaction on Wireless Communications, vol. 6, pp. 2004-2213, June 2007.

[59] V. Brik, E. Rozner, S. Banarjee, and P. Bahl, "DSAP: a Protocol for Coordinated Spectrum Aaccess," in Proc. IEEE 1st Symposium Dynamic Spectrum Access Networks, DySPAN'05, p. 611614, Nov. 2005.

[60] C. Raman, R. Yates, and N. Mandayam, "Scheduling Variable Rate Links Via a Spectrum Server," in Proc. IEEE 1st Symposium Dynamic Spectrum Access Networks, DySPAN'05, p. 110118, Nov. 2005.

[61] I. F. Akyildiz, J. Xie, and S. Mohanty, "A Survey of Mobility Management in Next-Generation All-IP-Based wireless system," IEEE Wireless communications, vol. 11, pp. 16-28, Aug 2004.

[62] J. Zhao, G. Zheng, and H. yang, "Distribuited coordination in dynamic spectrum allocation networks," in DySPAN 2005, First IEEE International Symposium onNew Frontiers in Dynamic Spectrum Access Networks, pp. 259-268, Nov 2005.

[63] Z. Chair and P. K. Varshney, "Optimal Data Fusion in Multiple Sensor Detection Systems," IEEE Trans. on Aerospace and Electronic Systems, vol. AES-22, pp. 98101, Jan 1986. 
[64] L. Ma, X. Han, and C.-C. Shen, "Dynamic Open spectrum Sharing MAC Protocol for Wireless ad hoc Network," in Proc. IEEE 1st Symposium Dynamic Spectrum Access Networks, DySPAN'05, p. 203213, Nov. 2005.

[65] K. R. Chowdhury and I. F. Akylidiz, "Cognitive Wireless Mesh Networks with Dynamic Spectrum Access," IEEE Jornal of Slected Areas in Communications, vol. 26 , pp. 168 - 181, Jan 2008.

[66] D. Cabric, S. Mishra, D. Wilkomm, R. Broderson, and A. Wolisz, "A Cognitive Radio Approach for Usage of Virtual Unlicensed Spectrum ," in Proc. IEEE Military Communications Conference, MILCOM'05, pp. 455-460, Atlantic City, NJ, USA, Oct 2005.

[67] M. Thoppian, S. Venkatesan, R. Prakash, and R. Chandrasekaran, "MAC-layer scheduling in cognitive radio based multi-hop wireless networks," in WoWMoM 2006, International Symposium on World of Wireless, Mobile and Multimedia Networks, p. 10 pp, Jun 2006.

[68] C. Guo, T. Zhang, Z. Zeng, and C. Feng, "Investigation on Spectrum Sharing Technology based on Cognitive Radio," in Proc. IEEE Int. Conf. on Communications and Networking, pp. 1-5, Beijing, China, Oct 2006.

[69] J. N. Laneman and G. W. Wornell, "Energy Efficient Antenna Sharing and Relaying for Wireless Networks," in IEEE WCNC'00, pp. 7-12, Chicago, IL, USA, Oct. 2000.

[70] M. O. Hasna and M.-S. Alouini, "End-to-End Performance of Transmisson Systems with Relays over Rayleigh-Fading Channels," IEEE Trans. on Wireless Communications, vol. 2, pp. 1126-1131, Nov. 2003.

[71] M. O. Hasna and M.-S. Alouini, "A Perfromance Study of Dual-Hop transmission with Fixed Gain Relays," IEEE Trans. on Wireless Communications, vol. 3, pp. 1963-1968, Nov. 2004. 
[72] C. de M. Cordeiro, K. S. Challapali, D. Birru, and S. S. N., "Ieee 802.22: An introduction to the first wireless standard based on cognitive radios," JCM, vol. 1, no. 1, pp. 38-47, 2006.

[73] S. M. Almalfouh and G. L. Stuber, "Uplink Resource Allocation in Cognitive Radio Networks with Imperfect Spectrum Sensing," in Proc. IEEE Veh. Tech. Conference, VTC'2010, pp. 1-6, Sep. 2010.

[74] J. N. Laneman, N. C. Tse, and G. W. Wornell, "Cooperative Diversity in Wireless Networks Efficient Protocols and Outage Behaviour," IEEE Trans. on Inf. Theory, vol. 50, pp. 3062-3080, Nov. 2004.

[75] E. Vistotisky, S. Kuffner, and R. Peterson, "On Collaborative Detection of TV Transmissions in Support of Dynamic Spectrum Sharing," in Proc. IEEE 1st Symposium Dynamic Spectrum Access Networks (DySPAN'05), pp. 338-345, Baltimore, MD, Nov. 2005.

[76] D. G. Brennan, "Linear Diversity Combining Techniques," Proc. IEEE, vol. 91, pp. 331-356, Feb 2003.

[77] T. Eng, N. Kong, and L. B. Milstein, "Comparison of Diversity Combining Techniques for Rayleigh-Fading Channels," IEEE Trans. on Communications, vol. 44, pp. 1117-1129, Sep. 1996.

[78] V. A. Aalo, "Performance of Maximal-ratio Diversity Systems in a Correlated Nakagami-Fading Environment," IEEE Trans. on Communications, vol. 43, pp. 2360-2369, Aug. 1995.

[79] T. Rappaport, Wireless Communications: Principles and Practice. (Prentice-Hall, 2nd Edition, NJ, USA, 2002).

[80] M. S. Alouini and A. J. Goldsmith, "A Unified Approach for Calculating the Error Rates of Linearly Modulated Signals over Genarlized Fading Channels," in Proc. 
IEEE Intl. Conference on communications, ICC'98, vol. COM-47, pp. 1324-1334, June 1998.

[81] H. Urkowitz, "Energy Detection of Unknown deterministic Signals," Proc. of IEEE, vol. 55, pp. 523-531, April 1967.

[82] J. Proakis, Digital communications. McGraw Hill, 3rd Edition, New York, NY, USA, 1995.

[83] A. Sahai, N. Hoven, and R. Tandra, "Some Fundamental Limits on Cognitive Radio," in Proc. of the Allerton Conference on Communication, Control, and Computing, 2004.

[84] W. A. Gardner and C. M. Spencer, "Signal Interception: Performance Advantage of Cyclic-Feature Detectors," IEEE Trans. on Communications, vol. 40, pp. 149-159, Jan. 1992.

[85] M. O. F. K. Jondral, "On the Extraction of the Channel Allocation Informationin Spectrum Pooling System," IEEE Journal on Selected Areas in Communications, vol. 25, pp. 558-565, Apr. 2007.

[86] J. Zhu, Z. Xu, F. Wang, B. Huang, and B. Zhang, "Double Threshold Energy Detection of Cooperative Spectrum Sensing in Cognitive Radio," in Proc. International Conference on Cognitive Radio Oriented Wireless Networks and Communications, CrownCom'08, pp. 1-5, Singapore, Singapore, May 2008.

[87] B. Canberk, I. F. Akyildiz, and S. Oktug, "Primary User Activity Modeling Using First-Difference Filter Clustering and Correlation in Cognitive Radio Networks," IEEE/ACM Trans. on Networking, no. 1, pp. 170-183, 2011.

[88] S. Shanker, C. Cordeiro, and K. Challapali, "Spectrum Agile Radios: Utilization and Sensing Archeticture," in Proc. IEEE International Symposium on New Fron- 
tiers in Dynamic Spectrum Access Networks, DySPAN, pp. 160-169, Baltimore, MD, USA, Nov 2005.

[89] Q. Li and D. Rus, "Global Clock Synchronization in Sensor Networks," IEEE Trans. on Computers, vol. 55, pp. 214-226, Feb 2006.

[90] L. Bao and J. J. Carcia-Luna-Aceves, "Topology Management in Ad Hoc Networks," in Proc. of the International Symposium on Mobile Ad Hoc Networking and Computing, pp. 129-140, Annapolis, MD, US, June 2003.

[91] M. Nakagami, The m-distribution: A General Formula of Intensity Distribution of Rapid Fading. Statistical Methods in Radio Wave Propagation. Pergamon, New York, USA, 1960.

[92] M. Abdel-Hafez and M. Safak, "Performance Analysis of Digital Cellular Systems in Nakagami Fading and Correlated Shadowing Environmental," IEEE Trans. on Vehicular Technology, vol. 48, pp. 1381-1391, Sept. 1999.

[93] R. Kwan and C. Leung, "General Order Selection Combining for Nakagami and Weibull Fading Channels," IEEE Trans. on Wireless Communications, vol. 6, pp. 2027-2033, June 2007.

[94] G. K. Karagiannidis, N. C. Sagias, and T. A. Tsiftsis, "Closed-Form Statistics for the sum of Squared Nakagami- $m$ Variates and its Applications," IEEE Trans. on Communications, vol. 54, pp. 1353-1359, Aug. 2004.

[95] M.-S. Alouni, A. Abdi, and M. Kaveh, "Sum of Gamma Variates and Performance of Wireless Communication Systems over Nakagami-Fading Channels," IEEE Trans. on Vehicular Technology, vol. 50, pp. 1471-1480, Nov. 2001.

[96] Q. T. Zhang, "Maximal-Ratio Combining over Nakagami Fading Channels with an Arbitrary ranch Covariance Matrix," IEEE Trans. on Vehicular Technology, vol. 48, pp. 1141-1150, July 1999. 
[97] H. Suzuki, "A Statistical Model for the Urban Radio Propagation," IEEE Trans. on Communications, vol. Com-25, pp. 673-680, July 1997.

[98] Q. T. Zhang, "Exact Analysis of Postdetection Combining for DPSK and NFSK Systems over Arbitrarily Correlated Nakagami Channels," IEEE Trans. on Communications, vol. 46, pp. 1459-1467, Nov. 1998.

[99] E. K. Al-Hussaini and A. A. M. Al-Bassiouni, "Performance of MRC Diversity Systems for the Detection of Signals with Nakagami Fading," IEEE Trans. on Communications, vol. COM-33, pp. 1315-1319, Dec. 1985.

[100] V. A. Aalo, "Performance of MRC Diversity Systems in a Correlated Nakagami Fading Environment," IEEE Trans. on Communications, vol. 43, pp. 2360-2369, Aug. 1995.

[101] A. M. Mathai, "Storage Capacity of a dam with Gamma Type Inputs," Ann. Inst. Math., vol. 34, no. 3, pp. 591-570, 1982.

[102] P. G. Moschopoulos, "The Distribution of the Sum of Independent Gamma Random Variables," Ann. Inst. Statist. Math. (Part A), vol. 37, pp. 541-544, 1985.

[103] X. Qin and R. A. Berry, "Distributed Approaches for Exploiting Multiuser Diversity in Wireless Networks," IEEE Trans. Information Theory, vol. 52, pp. 392-413, Feb. 2006.

[104] H. Yomo and E. de Carvalho, "A CSI Estimation Method for Wireless Relay Network," IEEE Communications Letters, vol. 11, pp. 480-482, June 2007.

[105] I. S. Gradshteyn and I. M. Ryzhik, Table of Integrals, Series and Products. Academic Press, 6th Edition, London, UK, 2000.

[106] M. Abramowitz and I. A. Stegun, Handbook of Mathematical Functions with Formulas, Graphs, and Mathematical Tables. Dover Press, 9th Edition, New York, USA, 1972. 
[107] C. Stevenson, G. Chouinard, Z. Lei, W. Hu, S. Shellhammer, and W. Caldwell, "IEEE 802.22: The First Cognitive Radio Wireless Regional Area Network Standard ," IEEE Communications Magazine, vol. 47, pp. 130-138, Jan. 2009.

[108] D. S. Michalopoulos and G. K. Karagiannidis, " Performance Analysis of single Relay selection in Rayleigh Fading," IEEE Trans. on Wireless Communications, vol. 7, pp. 3718-3724, Oct. 2008.

[109] T. Sauter, "Computation of Irregularly Oscillating Integrals," Applied Numerical Math, vol. 35, pp. 245-264, Nov. 2000.

[110] P. Wynn, "Acceleration Techniques in Numerical Analysis, with Particular Reference to Problems in One Independent Variable," in IFIPS, pp. 149-156, Munich 1962.

[111] S. Althunibat, R. Palacios, and F. Granelli, "Performance Optimisation of Soft and Hard Spectrum Sensing Schemes in Cognitive Radio," IEEE Communications Letters, vol. 16, pp. 998-1001, July 2012.

[112] A. H. Nuttall, "Some Integrals Involving the $Q_{m}$ Function," Naval Underwater systems center (NUSC) Technical Report, April 1972.

[113] S. Hussain and X. Fernando, "Cooperative Cognitive Radio Networks: New Approach for Detection Accuracy Analysis under Impaired Channels," Wireless Personal Communications-Springer, vol. 71, pp. 1755-1775, Aug. 2013.

[114] M. D. Renzo, L. Imbriglio, F. Grazios, and F. Santucci, "A Comprehensive Framework for Performance Analysis of Dual-Hop Cooperative Wireless Systems with Fixed-Gain Relays over Generalized Fading Channels," IEEE Trans. on Wireless Communications, vol. 8, pp. 5060-5074, Oct. 2009.

[115] A. Papoulis and S. U. Pillai, Probability, Random Variables, and Stochastic Processes. McGraw-Hill, 4th Edition, New York, USA, 2002. 
[116] Z. Wang and G. B. Giannakis, "A simplified and General Parameterization Quantifying Performance in Fading channels," IEEE Trans. on Communications, vol. 5, pp. 1389-1398, Aug. 2003.

[117] C. R. Lin and M. Gerla, "A Daptive Clustering for Mobile Wireless Networks," IEEE Journal on Selected Areas in Communications, vol. 15, pp. 1265-1273, Sep. 1997.

[118] E. Minieka, Optimization Algorithms for Networks and Graphs. 2nd ed., New York, USA, Marcel Dekker Inc., 1978.

[119] W. B. Heinzelman, A. P. Chandrakasan, and H. Balakrishnan, "An Application specific Protocol Architecture for Wireless Microsensor Networks," IEEE Trans. on Wireless Communications, vol. 1, no. 4, pp. 660-669, 2002.

[120] Z. Quan, A. Subramanian, and A. H. Sayed, "REACA: An Efficient Protocol Architecture for Large Scale Sensor Networks," IEEE Trans. on Wireless communications, vol. 6, pp. 2924-2933, Aug 2007.

[121] R. J. Vaughan and W. N. Venables, "Permanant Expressions for Order Statistics Densities," J. Roy. Statist. Soc. B., vol. 34, pp. 308-310, 1972.

[122] R. Kwan and C. Leung, "Selection Diversity in Non-Identically Distributed NakagamiFading Channels," in Proc. IEEE Sarnoff Symposium, New Jersy, April 2005.

[123] G. B. Arfken and H. J. Weber, Mathematical Methods for Physicists. Elsevier Academic Press, 6th Edition, Burlington, MA, USA, 2005.

[124] A. H. Nuttall, "Some Integrals Involving the $Q_{m}$ Function," Naval Underwater systems center (NUSC) Technical Report, Jan. 1975. 